\title{
Estimation in Semiparametric Quantile Factor Models
}

\author{
Shujie Ma \\ Oliver Linton \\ University of California at Riverside* University of Cambridge \\ Jiti Gao \\ Monash University ${ }^{\dagger}$ \\ August 27, 2018
}

\begin{abstract}
We propose an estimation methodology for a semiparametric quantile factor panel model. We provide tools for inference that are robust to the existence of moments and to the form of weak cross-sectional dependence in the idiosyncratic error term. We apply our method to daily stock return data.
\end{abstract}

Keywords: Cross-Sectional Dependence; Fama-French Model; Inference; Sieve Estimation

JEL classification: C14; C21; C23; G12

\footnotetext{
*Ma's research was partially supported by NSF grants DMS 1306972 and DMS 1712558.

†Gao's research was supported by the Australian Research Council Discovery Grants Program for its support under Grant numbers: DP150101012 \& DP170104421.
} 


\section{Introduction}

Factor models are widely used to capture the co-movement of a large number of time series and to model covariance matrices. They provide useful dimensionality reduction in many applications from climate modelling to finance. Perhaps the current state of the art for factor modelling is Fan, Liao, and Micheva (2013), which allowed the idiosyncratic covariance matrix to be non-diagonal but sparse, and used thresholding techniques (Cai and Liu, 2011) to impose sparsity and thereby obtain a better estimator of the covariance matrix and its inverse in this big-data setting. The usual approach ignores covariate information that can sometimes be informative. Connor, Hagmann and Linton (2012) developed a semiparametric factor regression methodology that introduces covariate information into the factor loading parameters. This model is well motivated in finance applications where it can be understood as a properly formulated version of the popular Fama-French (1992) approach to modelling returns with observable characteristics. The model also makes sense in other contexts where covariate information is available. Their application was to monthly stock returns, which is where the finance literature was focussed. Moreover, Fan, Liao and Wang (2016) proposed a Projected-PCA approach which employs principal component analysis to the projected data matrix onto a linear space spanned by covariates. It is worth noting that most existing works in the literature of factor models require at least four moments to establish their theoretical properties. See, for instance, Bai and Ng (2002), Bai and Li (2012), Lam and Yao (2012), Connor, Hagmann and Linton (2012), Fan, Liao, and Micheva (2013), Fan, Liao and Wang (2016), Li et al. (2017), among others. This may not be a binding restriction for monthly stock returns, but for daily stock returns this is a bit strong.

Quantile methods are widely used in statistics. They have the advantage of being robust to large observations. They can also provide more information about the conditional distribution away from the centre, which is relevant in many applications. In this paper, we propose estimation and inferential methodology for the quantile version of the Connor, Hagmann and Linton (2012) model. Our contribution is summarized as follows.

First, we propose an estimation algorithm for this model. We use sieve techniques to obtain preliminary estimators of the nonparametric beta functions, see Chen (2011) for a review, and use these to estimate the factor return vector at each time period. We then update the loading functions and factor returns sequentially. We compute the estimator in two steps for computational reasons. We have $J \times T$ unknown factor return parameters as well as $J \times K_{N}$ sieve parameters to estimate, and simultaneous estimation of these parameters without penalization would be challenging. Penalization of the factor returns here is not well motivated so we do not pursue this. Instead we first estimate the unrestricted additive quantile regression function for each time period and then impose the factor structure in a 
sequential fashion.

Second, we derive the limiting properties of our estimated factor returns and factor loading functions under the assumption that the included factors all have non zero mean and under weak conditions on cross-section and temporal dependence. A key consideration in the panel modelling of stock returns is what position to take on the cross sectional dependence in the idiosyncratic part of stock returns. Early studies assumed iid in the cross section, but this turns out to be not necessary. More recent work has allowed for cross sectional dependence in a variety of ways. Connor, Hagmann and Linton (2012) imposed a known industry cluster/block structure where the number of industries goes to infinity as do the number of members of the industry. Under this structure one obtains a CLT and inference can be conducted by estimating only the intra block covariances. Robinson and Thawornkaiwong (2012) considered a linear process structure driven by independent shocks. Dong, Gao and Peng (2015) introduced a spatial mixing structure to accommodate both serial correlation and cross-sectional dependence for a general panel data setting. Conley (1999) studied that under a lattice structure or some observable or estimable distance function that determines the ordering, one can consistently estimate the asymptotic covariance matrix. However, this type of structure is hard to justify for stock returns, and in that case their approach does not deliver consistent inference. Connor and Koraczyck (1993) considered a different cross-sectional dependence structure, namely, they supposed that there was an ordering of the cross sectional units such that weak dependence of the alpha mixing variety was held. They do not assume knowledge of the ordering as this was not needed for their main results. We adopt and generalize their structure. In fact, we allow for weak dependence simultaneously in the cross-section and time series dependence. This structure affects the limiting distribution of the estimated factor returns in a complicated fashion, and the usual Newey-West type of standard errors can't be adapted to account for the cross-sectional dependence here because the ordering is not assumed to be known. To conduct inference we have to take account of the correlation structure. We use the so-called fix-b asymptotics to achieve this, namely, we construct a test statistic based on an inconsistent fixed-b estimator of the correlation structure, as in Kiefer and Vogelsang (2002), and show that it has a pivotal limiting distribution that is a functional of a Gaussian process.

Third, our estimation procedure only requires that the time series mean of factor returns be non zero. A number of authors have noted that in the presence of a weak factor, regression identification strategies can break down (Bryzgalova, 2015). In view of this we provide a test of whether a given factor is present or not in each time period.

Fourth, we apply our procedure to CRSP daily data and show how the factor loading functions vary nonlinearly with state. The median regression estimators are comparable to 
those of Connor, Hagmann and Linton (2012) and can be used to test asset pricing theories under comparable quantile restrictions, see for example, Bassett, Koenker and Kordas (2004), and to design investment strategies. The lower quantile estimators could be used for risk management purposes. The advantage of the quantile method is its robustness to heavy tails in the response distribution, which may be present in daily data. Indeed our theory does not require any moment conditions.

The organization of this paper is given as follows. Section 2 proposes the main model and then discusses some identification issues. An estimation method based on B-splines is then proposed in Section 3. Section 4 establishes an asymptotic theory for the proposed estimation method. Section 5 discusses a covariance estimation problem and then considers testing for the factors involved in the main model. Section 6 gives an empirical application of the proposed model and estimation theory to model the dependence of daily returns on a set of characteristic variables. Section 7 concludes the paper with some discussion. All the mathematical proofs of the main results are given in an appendix and on-line supplemental materials.

\section{The model and identification}

We introduce some notations which will be used throughout the paper. For any positive numbers $a_{n}$ and $b_{n}$, let $a_{n} \asymp b_{n}$ denote $\lim _{n \rightarrow \infty} a_{n} / b_{n}=c$, for a positive constant $c$, and let $a_{n} \gg b_{n}$ denote $a_{n}^{-1} b_{n}=o(1)$. For any vector $\mathbf{a}=\left(a_{1}, \ldots, a_{n}\right)^{\top} \in \mathbb{R}^{n}$, denote $\|\mathbf{a}\|=\left(\sum_{i=1}^{n} a_{i}^{2}\right)^{1 / 2}$. For any symmetric matrix $\mathbf{A}_{s \times s}$, denote its $L_{2}$ norm as $\|\mathbf{A}\|=\max _{\zeta \in \mathbb{R}^{s}, \zeta \neq \mathbf{0}}\|\mathbf{A} \zeta\|\|\zeta\|^{-1}$. We use $(N, T) \rightarrow \infty$ to denote that $N$ and $T$ pass to infinity jointly.

We consider the following model for the $\tau^{\text {th }}$ conditional quantile function of the response $y_{i t}$ for the $i^{\text {th }}$ asset at time $t$ given as

$$
Q_{y_{i t}}\left(\tau \mid X_{i}, f_{t}\right)=f_{u t}+\sum_{j=1}^{J} g_{j}\left(X_{j i}\right) f_{j t}
$$

i.e., we suppose that

$$
y_{i t}=f_{u t}+\sum_{j=1}^{J} g_{j}\left(X_{j i}\right) f_{j t}+\varepsilon_{i t},
$$

for $i=1, \ldots, N$ and $t=1, \ldots, T$, where $y_{i t}$ is the excess return to security $i$ at time $t$; $f_{u t}$ and $f_{j t}$ are factor returns, which are unobservable; $g_{j}\left(X_{j i}\right)$ are the factor betas, which are unknown but smooth functions of $X_{j i}$, where $X_{j i}$ are observable security characteristics, and $X_{j i}$ lies in a compact set $\mathcal{X}_{j i}$. Let $X_{i}=\left(X_{1 i}, \ldots, X_{J i}\right)^{\top}$ and $f_{t}=\left(f_{u t}, f_{1 t}, \ldots, f_{J t}\right)^{\top}$. The error terms $\varepsilon_{i t}$ are the asset-specific or idiosyncratic returns and they satisfy that the conditional $\tau^{\text {th }}$ quantile of $\varepsilon_{i t}$ given $\left(X_{i}, f_{t}\right)$ is zero. The factors $f_{u t}$ and $f_{j t}$ and the factor betas $g_{j}(\cdot)$ should be $\tau$ specific. For notational simplicity, we suppress the $\tau$ subscripts. For model identifiability, we assume that: 
Assumption A0. For some probability measures $P_{j}$ we have $\int g_{j}\left(x_{j}\right) d P_{j}\left(x_{j}\right)=0$ and $\int\left(g_{j}\left(x_{j}\right)\right)^{2} d P_{j}\left(x_{j}\right)=1$ for all $j=1, \ldots, J$. Furthermore, $\liminf _{T \rightarrow \infty}\left|\sum_{t=1}^{T} f_{j t} / T\right|>0$ for each $j$.

The case where $\tau=1 / 2$ corresponds to the conditional median, and is comparable to the conditional mean model used in Connor, Hagmann and Linton (2012). The advantage of the median over the mean is its robustness to heavy tails and outliers, which is especially important with daily data. The case where $\tau=0.01$, say, might be of interest for the purposes of risk management, since this corresponds to a standard Value-at-Risk threshold in which case (2.1) gives the conditional Value-at-Risk given the characteristics and the factor returns at time $t$. To obtain an ex-ante measure we should have to employ a forecasting model for the factor returns.

Suppose that the $\tau^{\text {th }}$ conditional quantile function $Q_{y_{i t}}\left(\tau \mid X_{i}=x\right)$ of the response $y_{i t}$ at time $t$ given the covariate $X_{i}=x$ is additive

$$
H_{t}(\tau \mid x)=h_{u t}+\sum_{j=1}^{J} h_{j t}\left(x_{j}\right)
$$

where $h_{j t}(\cdot)$ are unknown functions without loss of generality satisfying $\int h_{j t}\left(x_{j}\right) d P_{j}\left(x_{j}\right)=0$ for $t=1, \ldots, T$ (Horowitz and Lee, 2005). Under the factor structure (2.1), we have for all j

$$
\int\left(\frac{1}{T} \sum_{t=1}^{T} h_{j t}\left(x_{j}\right)\right)^{2} d P_{j}\left(x_{j}\right)=\int g_{j}\left(x_{j}\right)^{2} d P_{j}\left(x_{j}\right) \times\left(\frac{1}{T} \sum_{t=1}^{T} f_{j t}\right)^{2}=\left(\frac{1}{T} \sum_{t=1}^{T} f_{j t}\right)^{2} .
$$

Provided $\sum_{t=1}^{T} f_{j t} \neq 0$, we can identify $g_{j}\left(x_{j}\right)$ by

$$
g_{j}\left(x_{j}\right)=\frac{\frac{1}{T} \sum_{t=1}^{T} h_{j t}\left(x_{j}\right)}{\sqrt{\int\left(\frac{1}{T} \sum_{t=1}^{T} h_{j t}\left(x_{j}\right)\right)^{2} d P_{j}\left(x_{j}\right)}} .
$$

We will use this as the basis for the proposal of the estimation method in Section 3 below.

\section{Estimation}

\subsection{Factor returns and characteristic-beta functions}

We propose an iterative algorithm to estimate the factor returns and the characteristic-beta functions. The algorithm makes use of the structure in (2.2) so that it circumvents the "curse of dimensionality" (Bellman, 1961) while retaining flexibility of the nonparametric regression. The right hand side of (2.1) is bilinear in unknown quantities, so it seems difficult to avoid such an algorithmic approach. 
To estimate $g_{j}(\cdot)$, we first approximate them by B-spline functions described as follows. Let $b_{j}\left(x_{j}\right)=\left\{b_{j, 1}\left(x_{j}\right), \ldots, b_{j, K_{N}}\left(x_{j}\right)\right\}^{\top}$ be a set of normalized B-spline functions of order $m$ (see, for example, de Boor (2001)), where $K_{N}=L_{N}+m$, and $L_{N}$ is the number of interior knots satisfying $L_{N} \rightarrow \infty$ as $N \rightarrow \infty$. We adopt the centered B-spline basis functions $B_{j}\left(x_{j}\right)=\left\{B_{j, 1}\left(x_{j}\right), \ldots, B_{j, K_{N}}\left(x_{j}\right)\right\}^{\top}$, where

$$
B_{j k}\left(x_{j}\right)=\sqrt{K_{N}}\left[b_{j, k}\left(x_{j}\right)-N^{-1} \sum_{i=1}^{N} b_{j, k}\left(X_{j i}\right)\right],
$$

so that $N^{-1} \sum_{i=1}^{N} B_{j k}\left(X_{j i}\right)=0$ and $\operatorname{var}\left\{B_{j k}\left(X_{j}\right)\right\} \asymp 1$. We first approximate the unknown functions $g_{j}\left(x_{j}\right)$ by B-splines such that $g_{j}\left(x_{j}\right) \approx B_{j}\left(x_{j}\right)^{\top} \boldsymbol{\lambda}_{j}$, where $\boldsymbol{\lambda}_{j}=\left(\lambda_{j, 1}, \ldots, \lambda_{j, K_{N}}\right)^{\top}$ are spline coefficients. Hence $N^{-1} \sum_{i=1}^{N} B_{j}\left(X_{j i}\right)^{\top} \boldsymbol{\lambda}_{j}=0$. Denote $f_{t}=\left\{f_{u t},\left(f_{j t}, 1 \leq j \leq J\right)^{\top}\right\}^{\top}$. Let $\boldsymbol{\lambda}=\left(\boldsymbol{\lambda}_{1}^{\top}, \ldots, \boldsymbol{\lambda}_{J}^{\top}\right)^{\top}$ and let $\rho_{\tau}(u)=u(\tau-I(u<0))$ be the quantile check function. The iterative algorithm is described as follows:

1. Find the initial estimates $\widehat{f}^{[0]}$ and $\widehat{g}_{j}^{[0]}(\cdot)$.

2. For given $\widehat{f}^{[i]}$, we obtain

$$
\widehat{\boldsymbol{\lambda}}^{[i+1]}=\arg \min _{\boldsymbol{\lambda} \in \mathbb{R}^{J K_{N}}} \sum_{i=1}^{N} \sum_{t=1}^{T} \rho_{\tau}\left(y_{i t}-\widehat{f}_{u t}^{[i]}-\sum_{j=1}^{J} B_{j}\left(X_{j i}\right)^{\top} \boldsymbol{\lambda}_{j} \widehat{f}_{j t}^{[i]}\right) .
$$

Let $\widehat{g}_{j}^{*[i+1]}\left(x_{j}\right)=B_{j}\left(x_{j}\right)^{\top} \widehat{\boldsymbol{\lambda}}_{j}^{[i+1]}$. The estimate for $g_{j}\left(x_{j}\right)$ at the $(i+1)^{\text {th }}$ step is

$$
\widehat{g}_{j}^{[i+1]}\left(x_{j}\right)=\frac{\widehat{g}_{j}^{*[i+1]}\left(x_{j}\right)}{\sqrt{N^{-1} \sum_{i=1}^{N} \widehat{g}_{j}^{*[i+1]}\left(X_{j i}\right)^{2}}} .
$$

3. For given $\widehat{g}_{j}^{[i+1]}\left(x_{j}\right)$, we obtain for $t=1, \ldots, T$

$$
\widehat{f}_{t}^{[i+1]}=\arg \min _{f_{t} \in \mathbb{R}^{J+1}} \sum_{i=1}^{N} \rho_{\tau}\left(y_{i t}-f_{u t}-\sum_{j=1}^{J} \widehat{g}_{j}^{[i+1]}\left(X_{j i}\right) f_{j t}\right) .
$$

We repeat steps 2 and 3 , and consider that the algorithm converges at the $(i+1)^{\text {th }}$ step when $\left\|\widehat{f}^{[i+1]}-\widehat{f}^{[i]}\right\|<\epsilon$ and $\left\|\widehat{\lambda}^{[i+1]}-\widehat{\lambda}^{[i]}\right\|<\epsilon$ for a small positive value $\epsilon$. Then the final estimates are $\widehat{f}_{t}=\widehat{f}_{t}^{[i+1]}$ and $\widehat{g}_{j}\left(x_{j}\right)=\widehat{g}_{j}^{[i+1]}\left(x_{j}\right)$. Our experience in numerical analysis suggests that the proposed method converges well and rapidly using the consistent initial values proposed in Section 3.2. The algorithm stops after a finite number of iterations by using the consistent initial values.

\subsection{Initial estimators}

We first approximate the unknown functions $h_{j t}\left(x_{j}\right)$ by B-splines such that $h_{j t}\left(x_{j}\right) \approx B_{j}\left(x_{j}\right)^{\top} \boldsymbol{\theta}_{j t}$, where $\boldsymbol{\theta}_{j t}=\left(\theta_{j t, 1}, \ldots, \theta_{j t, K_{N}}\right)^{\top}$ are spline coefficients. Let $\boldsymbol{\theta}_{t}=\left(\boldsymbol{\theta}_{1 t}^{\top}, \ldots, \boldsymbol{\theta}_{J t}^{\top}\right)^{\top}$. Then the estimators $\left(\widetilde{h}_{u t}, \widetilde{\boldsymbol{\theta}}_{t}^{\top}\right)^{\top}$ of $\left(h_{u t}, \boldsymbol{\theta}_{t}^{\top}\right)^{\top}$ are obtained by minimizing

$$
\sum_{i=1}^{N} \rho_{\tau}\left(y_{i t}-h_{u t}-\sum_{j=1}^{J} B_{j}\left(X_{j i}\right)^{\top} \boldsymbol{\theta}_{j t}\right)
$$


with respect to $\left(h_{u t}, \boldsymbol{\theta}_{t}^{\top}\right)^{\top} \in \mathbb{R}^{J K_{N}+1}$. As a result, the estimator of $h_{j t}\left(x_{j}\right)$ is $\widetilde{h}_{j t}\left(x_{j}\right)=$ $B_{j}\left(x_{j}\right)^{\top} \widetilde{\boldsymbol{\theta}}_{j t}$. We then obtain the initial estimators of $g_{j}\left(x_{j}\right)$

$$
\widehat{g}_{j}^{[0]}\left(x_{j}\right)=\frac{T^{-1} \sum_{t=1}^{T} \widetilde{h}_{j t}\left(x_{j}\right)}{\sqrt{N^{-1} \sum_{i=1}^{N}\left(\frac{1}{T} \sum_{t=1}^{T} \widetilde{h}_{j t}\left(X_{j i}\right)\right)^{2}}} .
$$

The initial estimator of $f_{t}$ is

$$
\widehat{f}_{t}^{[0]}=\arg \min _{f_{t} \in \mathbb{R}^{J+1}} \sum_{i=1}^{N} \rho_{\tau}\left(y_{i t}-f_{u t}-\sum_{j=1}^{J} \widehat{g}_{j}^{[0]}\left(X_{j i}\right) f_{j t}\right)
$$

for $t=1, \ldots, T$.

\section{Asymptotic theory of the estimators}

We suppose that there is some relabelling of the cross-sectional units $i_{l_{1}}, \ldots, i_{l_{N}}$, whose generic index we denote by $i^{*}$, such that the cross sectional dependence decays with the distance $\left|i^{*}-j^{*}\right|$. This assumption has been made in Connor and Korajczyk (1993) and Lee and Robinson (2016). Our estimation procedure does not need to know the ordering of the data. However, to develop a robust inference procedure that accounts for heteroscedasticity and cross-sectional correlation (HAC), we need to order the data across $i$. As discussed in Lee and Robinson (2016), in some economic applications, data may be ordered according to some explanatory variables. Such considerations are pursued in our real data analysis with detailed discussions given in Section 6, For notational simplicity, we denote the indices as $\{i, 1 \leq i \leq N\}$ after the ordering.

Let $g_{j}^{0}(\cdot)$ for $j=1, \ldots, J$ and $f_{t}^{0}=\left(f_{u t}^{0}, f_{1 t}^{0}, \ldots, f_{J t}^{0}\right)^{\top}$ be the true factor betas and factor returns in model (2.2). For model identifiability, assume $E\left\{g_{j}^{0}\left(X_{j i}\right)\right\}=0$ and $E\left\{g_{j}^{0}\left(X_{j i}\right)\right\}^{2}=$ 1. Let $\mathbb{N}$ denote the collection of all positive integers. We use a $\phi$-mixing coefficient to specify the dependence structure. Let $\left\{W_{i t}: 1 \leq i \leq N, 1 \leq t \leq T\right\}$, where $W_{i t}=\left(X_{i}^{\top}, f_{t}^{\top}, \varepsilon_{i t}\right)^{\top}$ and $\varepsilon_{i t}=y_{i t}-f_{u t}^{0}-\sum_{j=1}^{J} g_{j}^{0}\left(X_{j i}\right) f_{j t}^{0}$. For $S_{1}, S_{2} \subset[1, \ldots, N] \times[1, \ldots, T]$, let

$$
\phi\left(S_{1}, S_{2}\right) \equiv \sup \left\{|P(A \mid B)-P(A)|: A \in \sigma\left(W_{i t},(i, t) \in S_{1}\right), B \in \sigma\left(W_{i t},(i, t) \in S_{2}\right)\right\},
$$

where $\sigma(\cdot)$ denotes a $\sigma$-field. Then the $\phi$-mixing coefficient of $\left\{W_{i t}\right\}$ for any $k \in \mathbb{N}$ is defined as

$$
\phi(k) \equiv \sup \left\{\phi\left(S_{1}, S_{2}\right): d\left(S_{1}, S_{2}\right) \geq k\right\}
$$

where

$$
d\left(S_{1}, S_{2}\right) \equiv \min \left\{\sqrt{|t-s|^{2}+|i-j|^{2}}:(i, t) \in S_{1},(j, s) \in S_{2}\right\}
$$

Without loss of generality, we assume that $\mathcal{X}_{j i}=[a, b]$. Denote $h_{t}^{0}(x)=\left\{h_{j t}^{0}\left(x_{j}\right), 1 \leq\right.$ $j \leq J\}^{\top}$, where $h_{j t}^{0}(\cdot)$ are the true unknown functions in $(2.3)$ and $x=\left(x_{1}, \ldots, x_{J}\right)^{\top}$. Let $G_{i}^{0}\left(X_{i}\right)=\left\{1, g_{1}^{0}\left(X_{1 i}\right), \ldots, g_{J}^{0}\left(X_{J i}\right)\right\}^{\top}$. We make the following assumptions. 
(C1) $\left\{W_{i t}\right\}$ is a random field of $\phi$-mixing random variables. The $\phi$-mixing coefficient of $\left\{W_{i t}\right\}$ satisfies $\phi(k) \leq K_{1} e^{-\lambda_{1} k}$ for $K_{1}, \lambda_{1}>0$. For each given $i,\left\{W_{i t}\right\}$ is a strictly stationary sequence.

(C2) The conditional density $p_{i}\left(\varepsilon \mid x_{i}, f_{t}\right)$ of $\varepsilon_{i t}$ given $\left(x_{i}, f_{t}\right)$ satisfies the Lipschitz condition of order 1 and $\inf _{1 \leq i \leq N, 1 \leq t \leq T} p_{i}\left(0 \mid x_{i}, f_{t}\right)>0$. For every $1 \leq j \leq J$, the density function $p_{X_{j i}}(\cdot)$ of $X_{j i}$ is bounded away from 0 and satisfies the Lipschitz condition of order 1 on $[a, b]$. The density function $f_{X_{i}}(\cdot)$ of $X_{i}$ is absolutely continuous on $[a, b]^{J}$.

(C3) The functions $g_{j}^{0}$ and $h_{j t}^{0}$ are $r$-times continuously differentiable on its support for some $r>2$. The spline order satisfies $m \geq r$.

(C4) There exist some constants $0<c_{h} \leq C_{h}<\infty$ such that $c_{h} \leq\left(\frac{1}{T} \sum_{t=1}^{T} f_{j t}^{0}\right)^{2} \leq C_{h}$ for all $j$ with probability tending to one.

(C5) The eigenvalues of the $(J+1) \times(J+1)$ matrix $N^{-1} \sum_{i=1}^{N} E\left(G_{i}^{0}\left(X_{i}\right) G_{i}^{0}\left(X_{i}\right)^{\top}\right)$ are bounded away from zero.

(C6) Let $\Omega_{N}^{0}$ be the covariance matrix of $N^{-1 / 2} \sum_{i=1}^{N} G_{i}^{0}\left(X_{i}\right)\left(\tau-I\left(\varepsilon_{i t}<0\right)\right)$. The eigenvalues of $\Omega_{N}^{0}$ are bounded away from zero and infinity.

We allow that $\left\{W_{i t}\right\}$ are weakly dependent across $i$ and $t$, but need to satisfy the strong mixing condition given in Condition (C1). Moreover, Condition (C1) implies that $\left\{X_{i}\right\}$ is marginally cross-sectional mixing, and $\left\{f_{t}\right\}$ is marginally temporally mixing. Similar assumptions are used in Gao, Lu and Tjøstheim (2006) for an alpha-mixing condition in a spatial data setting, and Dong, Gao and Peng (2016) for introducing a spatial mixing condition in a panel data setting. Conditions (C2) and (C3) are commonly used in the nonparametric smoothing literature, see for example, Horowitz and Lee (2005), and Ma, Song and Wang (2013). Conditions (C4) and (C5) are similar to Conditions A2, A5 and A7 of Connor, Matthias and Linton (2012).

Define

$$
\Lambda_{N t}^{0}=N^{-1} \sum_{i=1}^{N} E\left\{p_{i}\left(0 \mid X_{i}, f_{t}\right) G_{i}^{0}\left(X_{i}\right) G_{i}^{0}\left(X_{i}\right)^{\top}\right\}
$$

and

$$
\Sigma_{N t}^{0}=\tau(1-\tau)\left(\Lambda_{N t}^{0}\right)^{-1} \Omega_{N}^{0}\left(\Lambda_{N t}^{0}\right)^{-1} .
$$

The theorem below presents the asymptotic distribution of the final estimator $\widehat{f}_{t}$. Define

$$
\phi_{N T}=\sqrt{K_{N} /(N T)}+K_{N}^{3 / 2} N^{-3 / 4} \sqrt{\log N T}+K_{N}^{-r} .
$$

Let $d_{N T}$ be a sequence satisfying

$$
d_{N T}=O\left(\phi_{N T}\right)
$$


Theorem 1. Assume that Conditions (C1)-(C5) hold, and $K_{N}^{4} N^{-1}=o(1), K_{N}^{-r+2}(\log T)=$ $o(1)$ and $K_{N}^{-1}(\log N T)(\log N)^{4}=o(1)$. Suppose that the algorithm in Section 3.1 converges within a finite number of iterations. Then, for any there is a stochastically bounded sequence $\delta_{N, j t}$ such that as $N \rightarrow \infty$,

$$
\sqrt{N}\left(\Sigma_{N t}^{0}\right)^{-1 / 2}\left(\widehat{f}_{t}-f_{t}^{0}-d_{N T} \delta_{N, t}\right) \stackrel{\mathcal{D}}{\rightarrow} \mathcal{N}\left(\mathbf{0}, \mathbf{I}_{J+1}\right)
$$

where $\delta_{N, t}=\left(\delta_{N, j t}, 0 \leq j \leq J\right)^{\top}, d_{N T}$ is given in 4.3), and $\mathbf{I}_{J+1}$ is the $(J+1) \times(J+1)$ identity matrix.

The next theorem establishes the rate of convergence of the final estimator $\widehat{g}_{j}\left(x_{j}\right)$.

Theorem 2. Suppose that the same conditions as given in Theorem 1 hold. Then, for each $j$,

$$
\left[\int\left\{\widehat{g}_{j}\left(x_{j}\right)-g_{j}^{0}\left(x_{j}\right)\right\}^{2} d x_{j}\right]^{1 / 2}=O_{p}\left(\phi_{N T}\right)+o_{p}\left(N^{-1 / 2}\right),
$$

where $\phi_{N T}$ is given in 4.2.).

Remark 1: The orders $\sqrt{K_{N} /(N T)}$ and $K_{N}^{-r}$ are from the noise and bias terms for nonparametric estimation, respectively, and the order $K_{N}^{3 / 2} N^{-3 / 4} \sqrt{\log N T}$ from the approximation of the Bahadur representation in the quantile regression setting. This says that if the order $K_{N} \asymp(N T)^{1 /(2 r+1)}$ is chosen, and $T=O\left(N^{\alpha}\right)$, where $\alpha<1 / 2-3 /(2 r+3)$, then the rate of $\phi_{N T}$ is $O_{P}\left((N T)^{-r /(2 r+1)}\right)$, which is optimal, see for example, Chen and Christensen (2015).

Remark 2: By using the asymptotic normality provided in Theorem 1, we can conduct inference for $f_{t}^{0}$ for each $t$, such as constructing the confidence interval. Note that in the asymptotic distribution in Theorem [1, there is a bias term $d_{N T} \delta_{N, t}$ involved. Let $K_{N} \asymp$ $(N T)^{1 /(2 r+1)}$ and $T \asymp N^{\alpha}$, where $1 /(2 r)<\alpha<1 / 2-3 /(2 r+3)$ and $r>3$. Then the asymptotic bias is negligible and thus we have

$$
\sqrt{N}\left(\Sigma_{N t}^{0}\right)^{-1 / 2}\left(\widehat{f}_{t}-f_{t}^{0}\right) \rightarrow \mathcal{N}\left(\mathbf{0}, \mathbf{I}_{J+1}\right)
$$

Remark 3. It is possible to develop inferential results for $g_{j}$ following Chen and Liao (2012) and Chen and Pouzo (2015). As is usual in nonparametric estimation, the weak crosssectional and temporal dependence does not affect the limiting distribution, and so standard techniques can be applied. In fact, one may conclude the estimation algorithm with a kernel step and demonstrate the oracle efficiency property, see Horowitz and Mammen (2011). 


\section{Covariance estimation and hypothesis testing for the factors}

In order to construct the confidence interval given in (4.5) we need to estimate $\Omega_{N}^{0}$ and $\Lambda_{N t}^{0}$, since they are unknown. For estimation of $\Lambda_{N t}^{0}$, if we use its sample analogue, the conditional density $p_{i}\left(0 \mid X_{i}, f_{t}\right)$ needs to be estimated. Instead of using this direct way, we use the Powell's kernel estimation idea in Powell (1991), and estimate $\Lambda_{N t}^{0}$ by

$$
\widehat{\Lambda}_{N t}=(N h)^{-1} \sum_{i=1}^{N} K\left(\frac{y_{i t}-\widehat{f}_{u t}-\sum_{j=1}^{J} \widehat{g}_{j}\left(X_{j i}\right) \widehat{f}_{j t}}{h}\right) \widehat{G}_{i}\left(X_{i}\right) \widehat{G}_{i}\left(X_{i}\right)^{\top},
$$

where $\widehat{G}_{i}\left(X_{i}\right)=\left\{1, \widehat{g}_{1}\left(X_{1 i}\right), \ldots, \widehat{g}_{J}\left(X_{J i}\right)\right\}^{\top}$, while $K(\cdot)$ is the uniform kernel $K(u)=2^{-1} I(|u| \leq$ $1)$ and $h$ is a bandwidth.

First, we show that the estimator $\widehat{\Lambda}_{N t}$ is a consistent estimator of $\Lambda_{N t}^{0}$ given in the theorem below.

Theorem 3. Suppose that the same conditions as given in Theorem 1 hold, and $h \rightarrow 0$, $h^{-1} \phi_{N T}=o(1), h^{-1} N^{-1 / 2}=O(1)$, where $\phi_{N T}$ is given in 4.2). Then, we have $\| \widehat{\Lambda}_{N t}-$ $\Lambda_{N t}^{0} \|=o_{p}(1)$.

Moreover, the exact form of $\Omega_{N}^{0}$ defined in Condition (C6) is given by

$$
\begin{aligned}
\Omega_{N}^{0} & =(N T)^{-1} \sum_{t=1}^{T} E\left[\left\{\sum_{i=1}^{N} G_{i}^{0}\left(X_{i}\right)\left(\tau-I\left(\varepsilon_{i t}<0\right)\right)\right\}\left\{\sum_{i=1}^{N} G_{i}^{0}\left(X_{i}\right)\left(\tau-I\left(\varepsilon_{i t}<0\right)\right)\right\}^{\top}\right] \\
& =\frac{\tau(1-\tau)}{N} \sum_{i=1}^{N} E\left\{G_{i}^{0}\left(X_{i}\right) G_{i}^{0}\left(X_{i}\right)^{\top}\right\}+(N T)^{-1} \sum_{t=1}^{T} \sum_{i \neq j}^{N} E\left(v_{i t} v_{j t}^{\top}\right),
\end{aligned}
$$

where $v_{i t}=G_{i}^{0}\left(X_{i}\right)\left(\tau-I\left(\varepsilon_{i t}<0\right)\right)$ for $i=1, \ldots, N$. To estimate $\Omega_{N}^{0}$, its sample analogue is not consistent. Kernel-based robust estimators that account for HAC are developed (Conley, 1999), and are shown to be consistent under a variety of sets of conditions. It requires to use a truncation lag or "bandwidth", which tends to infinity at a slower rate of $N$. As pointed out by Kiefer and Vogelsang (2005), this is a convenient assumption mathematically to ensure consistency, but it is unrealistic in finite sample studies. Adopting the idea in Kiefer and Vogelsang (2005), we let the bandwidth $M$ be proportional to the sample size $N$, i.e., $M=b N$ for $b \in(0,1]$, and then we derive the fixed-b asymptotics (Kiefer and Vogelsang; 2005) for the HAC estimator of $\Omega_{N}^{0}$ under the quantile setting. The HAC estimator is given as $\widehat{\Omega}_{N, M}=T^{-1} \sum_{t=1}^{T} \widehat{\Omega}_{N t, M}$, where

$$
\widehat{\Omega}_{N t, M}=\frac{\tau(1-\tau)}{N} \sum_{i=1}^{N} \widehat{G}_{i}\left(X_{i}\right) \widehat{G}_{i}\left(X_{i}\right)^{\top}+N^{-1} \sum_{i \neq j}^{N} K^{*}\left(\frac{i-j}{M}\right) \widehat{v}_{i t} \widehat{v}_{j t}^{\top},
$$

where $\widehat{v}_{i t}=\widehat{G}_{i}\left(X_{i}\right)\left(\tau-I\left(\widehat{\varepsilon}_{i t}<0\right)\right)$ for $i=1, \ldots, N, \widehat{\varepsilon}_{i t}=y_{i t}-\widehat{f}_{u t}-\sum_{j=1}^{J} \widehat{g}_{j}\left(X_{j i}\right) \widehat{f}_{j t}, K^{*}(u)$ is a symmetric kernel weighting function satisfying $K^{*}(0)=1$, and $\left|K^{*}(u)\right| \leq 1$, and $M$ 
trims the sample autocovariances and acts as a truncation lag. Consistency of $\widehat{\Omega}_{N, M}$ needs that $M \rightarrow \infty$ and $M / N \rightarrow 0$. The following theorem provides the limiting distribution of $\widehat{\Omega}_{N, M=b N}$ when $M=b N$ for $b \in(0,1]$.

Next, we will show asymptotic theory for the HAC covariance estimator under a sequence where the smoothing parameter $M$ equals to $b N$. Let $\Omega^{0}=\lim _{N \rightarrow \infty} \Omega_{N}^{0}$, and $\Omega^{0}$ can be written as $\Omega^{0}=\Upsilon \Upsilon^{\top}$, where $\Upsilon$ is a lower triangular matrix obtained from the Cholesky decomposition of $\Omega^{0}$.

Theorem 4. Suppose that the same conditions as given in Theorem 1 hold, and $\phi_{N T} N^{1 / 2}=$ $o(1)$, and $K^{* \prime \prime}(u)$ exists for $u \in[-1,1]$ and is continuous. Let $M=b N$ for $b \in(0,1]$. Then as $N \rightarrow \infty$,

$$
\widehat{\Omega}_{N, M=b N} \stackrel{\mathcal{D}}{\rightarrow} \Upsilon \int_{0}^{1} \int_{0}^{1}-\frac{1}{b^{2}} K^{* \prime \prime}\left(\frac{r-s}{b}\right) B_{J+1}(r) B_{J+1}(s)^{\top} d r d s \Upsilon^{\top},
$$

where $B_{J+1}(r)=W_{J+1}(r)-r W_{J+1}(1)$ denotes $a(J+1) \times 1$ vector of standard Brownian bridges, and $W_{J+1}(r)$ denotes a $(J+1)$-vector of independent standard Wiener processes where $r \in[0,1]$.

Theorem 4 establishes the limiting distribution of $\widehat{\Omega}_{N, M=b N}$, although $\widehat{\Omega}_{N, M=b N}$ is an inconsistent estimator of $\Omega^{0}$. However, it can be used to construct asymptotically pivotal tests involving $f_{t}^{0}$.

Consider testing the null hypothesis $H_{0}: R f_{t}^{0}=r$ against the alternative hypothesis $H_{1}$ : $R f_{t}^{0} \neq r$, where $R$ is a $q \times(J+1)$ matrix with rank $q$ and $r$ is a $q \times 1$ vector. We construct an $F$-type statistic given as

$$
F_{N t, b}=N\left(R \widehat{f}_{t}-r\right)^{\top}\left\{R \tau(1-\tau) \widehat{\Lambda}_{N t}^{-1} \widehat{\Omega}_{N, M=b N} \widehat{\Lambda}_{N t}^{-1} R^{\top}\right\}^{-1}\left(R \widehat{f}_{t}-r\right) / q .
$$

When $q=1$, we can construct a $t$-type statistic:

$$
T_{N t, b}=\frac{N^{1 / 2}\left(R \widehat{f}_{t}-r\right)}{\sqrt{\left.R \tau(1-\tau) \widehat{\Lambda}_{N t}^{-1} \widehat{\Omega}_{N, M=b N} \widehat{\Lambda}_{N t}^{-1}\right\}^{-1} R^{\top}}} .
$$

The limiting distributions of $F_{N t, b}$ and $T_{N t, b}$ under the null hypothesis are given in the following theorem.

Theorem 5. Suppose that the same conditions as given in Theorem 1 hold, and $\phi_{N T} N^{1 / 2}=$ $o(1)$, and $K^{* \prime \prime}(u)$ exists for $u \in[-1,1]$ and is continuous. Let $M=b N$ for $b \in(0,1]$. Then under the null hypothesis $H_{0}: R f_{t}^{0}=r$, as $N \rightarrow \infty$,

$$
F_{N t, b} \stackrel{\mathcal{D}}{\rightarrow}\{\tau(1-\tau)\}^{-1} W_{q}(1)^{\top}\left\{\int_{0}^{1} \int_{0}^{1}-\frac{1}{b^{2}} K^{* \prime \prime}\left(\frac{r-s}{b}\right) B_{q}(r) B_{q}(s)^{\top} d r d s\right\}^{-1} W_{q}(1) / q .
$$

If $q=1$, then as $N \rightarrow \infty$,

$$
T_{N t, b} \stackrel{\mathcal{D}}{\rightarrow} \frac{W_{1}(1)}{\sqrt{\tau(1-\tau)} \sqrt{\int_{0}^{1} \int_{0}^{1}-\frac{1}{b^{2}} K^{* \prime \prime}\left(\frac{r-s}{b}\right) B_{1}(r) B_{1}(s) d r d s}} .
$$


Let $\Lambda_{t}^{0}=\lim _{N \rightarrow \infty} \Lambda_{N t}^{0}$. The limiting distributions of $F_{N t, b}$ and $T_{N t, b}$ under the alternative hypothesis $H_{1}: R f_{t}^{0}=r+c N^{-1 / 2}$ are given in the following theorem.

Theorem 6. Let $\Upsilon_{t}^{*}=\left(R \Lambda_{t}^{-1} \Omega^{0} \Lambda_{t}^{-1} R^{\top}\right)^{1 / 2}$. Suppose that the same conditions as given in Theorem 11 hold, and $\phi_{N T} N^{1 / 2}=o(1)$, and $K^{* \prime \prime}(u)$ exists for $u \in[-1,1]$ and is continuous. Let $M=b N$ for $b \in(0,1]$. Then under the alternative hypothesis $H_{1}: R f_{t}^{0}=r+c N^{-1 / 2}$, as $N \rightarrow \infty$,

$$
\begin{aligned}
& F_{N t, b} \stackrel{\mathcal{D}}{\rightarrow}\{\tau(1-\tau)\}^{-1}\left\{\Upsilon_{t}^{*-1} c+W_{q}(1)\right\}^{\top} \times \\
& \left\{\int_{0}^{1} \int_{0}^{1}-\frac{1}{b^{2}} K^{* \prime \prime}\left(\frac{r-s}{b}\right) B_{q}(r) B_{q}(s)^{\top} d r d s\right\}^{-1}\left\{\Upsilon_{t}^{*-1} c+W_{q}(1)\right\} / q .
\end{aligned}
$$

If $q=1$, then as $N \rightarrow \infty$,

$$
T_{N t, b} \stackrel{\mathcal{D}}{\rightarrow} \frac{\Upsilon_{t}^{*-1} c+W_{1}(1)}{\sqrt{\tau(1-\tau)} \sqrt{\int_{0}^{1} \int_{0}^{1}-\frac{1}{b^{2}} K^{* \prime \prime}\left(\frac{r-s}{b}\right) B_{1}(r) B_{1}(s) d r d s}} .
$$

Remark. If $K^{*}(x)$ is the Bartlett kernel, then

$$
\begin{gathered}
\int_{0}^{1} \int_{0}^{1}-\frac{1}{b^{2}} K^{* \prime \prime}\left(\frac{r-s}{b}\right) B_{q}(r) B_{q}(s)^{\top} d r d s \\
=\frac{2}{b} \int_{0}^{1} B_{q}(r) B_{q}(r)^{\top} d r-\frac{1}{b} \int_{0}^{1-b}\left\{B_{q}(r+b) B_{q}(r)^{\top}+B_{q}(r) B_{q}(r+b)^{\top}\right\} d r .
\end{gathered}
$$

These results allow one to test whether the factors are zero in a particular time period or not. Our tests are robust to the form of the cross-sectional dependence in the idiosyncratic error.

\section{Application}

In a series of important papers, Fama and French (hereafter denoted FF), demonstrated that there have been large return premia associated with size and value, which are observable characteristics of stocks. They contended that these return premia can be ascribed to a rational asset pricing paradigm in which the size and value characteristics proxy for assets' sensitivities to pervasive sources of risk in the economy. FF (1993) used a simple portfolio sorting approach to estimating their factor model. Connor, Hagmann, and Linton (2012) used kernel-based semiparametric regression methodology to capture the same phenomenon.

In our data analysis, we use all securities from Center for Research in Security Prices (CRSP) which have complete daily return records from 2005 to 2013, and have two-digit Standard Industrial Classification code (from CRSP), market capitalization (from Compustat) and book value (from Compustat) records. We use daily returns in excess of the risk-free 
return of 347 stocks. We consider the same four characteristic variables as given in Connor, Hagmann and Linton (2012), and Fan, Liao and Wang (2016), which are size, value, momentum and volatility. Connor, Hagmann and Linton (2012) provided some detailed descriptions of these characteristics. They are calculated using the same method as described in Fan, Liao and Wang (2016).

We fit the quantile factor model (2.1) for each year, so that there are $T=251$ observations. By taking the same strategy as He and Shi (1996), we select the number of interior knots $L_{N}$ by minimizing the Bayesian information criterion (BIC) given as

$$
\operatorname{BIC}\left(L_{N}\right)=\log \left\{(N T)^{-1} \sum_{i=1}^{N} \sum_{t=1}^{T} \rho_{\tau}\left(y_{i t}-\widehat{f}_{u t}-\sum_{j=1}^{J} \widehat{g}_{j}\left(X_{j i}\right) \widehat{f}_{j t}\right)\right\}+\frac{\log (N T)}{2 N T} J\left(L_{N}+m\right) .
$$

For the estimator $\widehat{\Lambda}_{N t}$ given in (5.1), the optimal order for the bandwidth $h$ is in the order of $N^{-1 / 5}$. We let $h=\kappa N^{-1 / 5}$ in our numerical analysis and take different values for $\kappa$. For the estimator $\widehat{\Omega}_{N t, M=b N}$ given in (5.2), we use different values for $b$, and use the Bartlett kernel as suggested in Kiefer and Vogelsang (2005).

Figures 1, 3 show the plots of the four estimated loading functions for the year of 2009, 2010, 2011, and 2012 at different quantiles $\tau=0.2,0.5$ and 0.8. We observe that the estimated loading functions have similar shapes for these four years. Moreover, for the size, value and momentum characteristics, the estimated functions show a clear nonlinear pattern, and at different quantiles, the curves are different for the same characteristic. For example, for the size characteristic, the estimated loading function fluctuates around zero and it has a sharp drop after the value of size variable exceeds certain value at the quantiles $\tau=0.2$ and 0.8. However, it has a smooth decreasing pattern for the median with $\tau=0.5$. For the momentum characteristic, the estimated function shows different curves at the three quantiles.

Next, we let $\kappa=0.5,1,1.5$ and $b=0.2,0.4,0.6$, respectively, for calculation of $\widehat{\Lambda}_{N t}$ and $\widehat{\Omega}_{N t, M=b N}$. For obtaining the robust estimator $\widehat{\Omega}_{N t, M=b N}$, the data need to be ordered across $i$. We consider two different orderings. First, we take the same strategy as Lee and Robinson (2016) by ordering the data according to firm size, since firms of similar size may be subject to similar shocks. Second, we use the information of the four explanatory variables by ordering the data according to the first principal component of the covariate matrix. Using the year of 2012, we test for the statistical significance of each factor at each time point, based on the proposed $t$-type statistic and its distribution given in Theorem 5 . Then for each factor, we find the percentage of the $t$-type statistics that are significant at a $95 \%$ confidence level across the 251 time periods. Based on the two different ordering strategies, Tables 1 and 2, respectively, show the annualized standard deviations of the factor returns, the percentage of significant $t$-type statistics for each factor, and the median $\mathrm{p}$-value at $\tau=0.5$. We can see that the results are consistent for different values of $\kappa$ and $b$ and for the two different 
Figure 1: The plots of the estimated loading functions for the year of 2009 (dotted-dashed red lines), 2010 (dotted magenta lines), 2011 (dashed blue lines), and 2012 (solid black lines) at $\tau=0.2$.

Size Characteristics, $\tau=0.2$

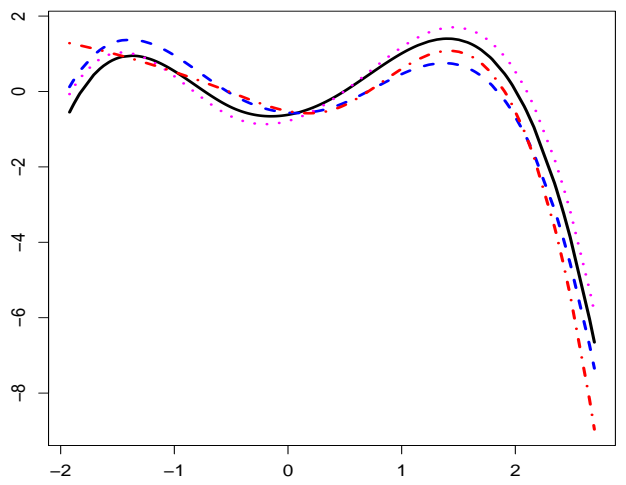

Momentum Characteristics, $\tau=0.2$

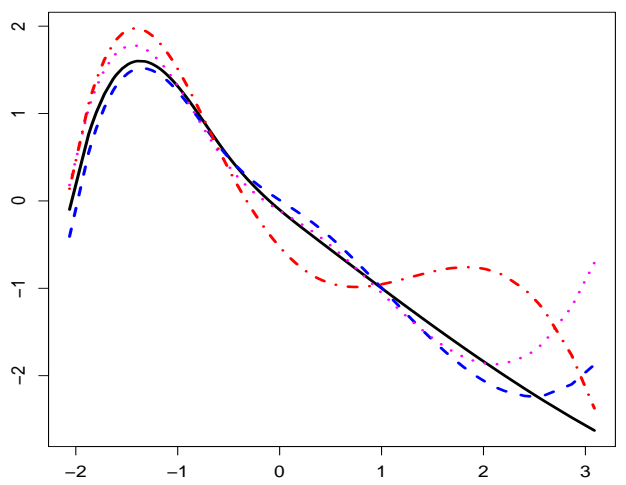

Value Characteristics, $\tau=0.2$

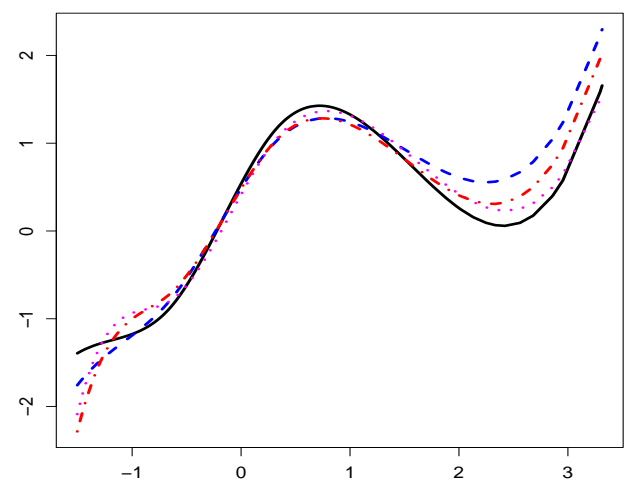

Volatility Characteristics, $\tau=0.2$

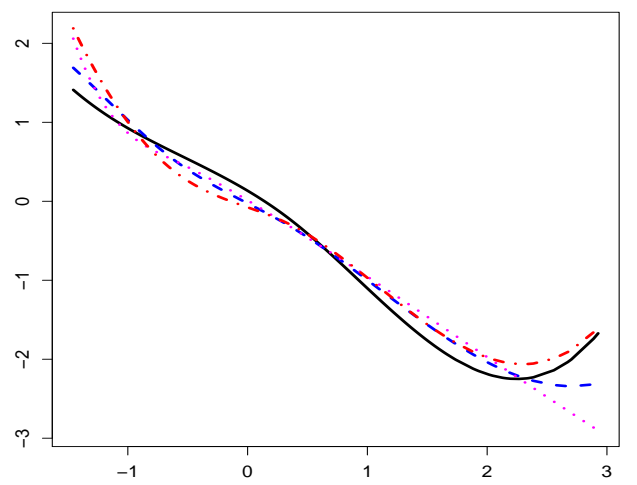


Figure 2: The plots of the estimated loading functions for the year of 2009 (dotted-dashed red lines), 2010 (dotted magenta lines), 2011 (dashed blue lines), and 2012 (solid black lines) at $\tau=0.5$.
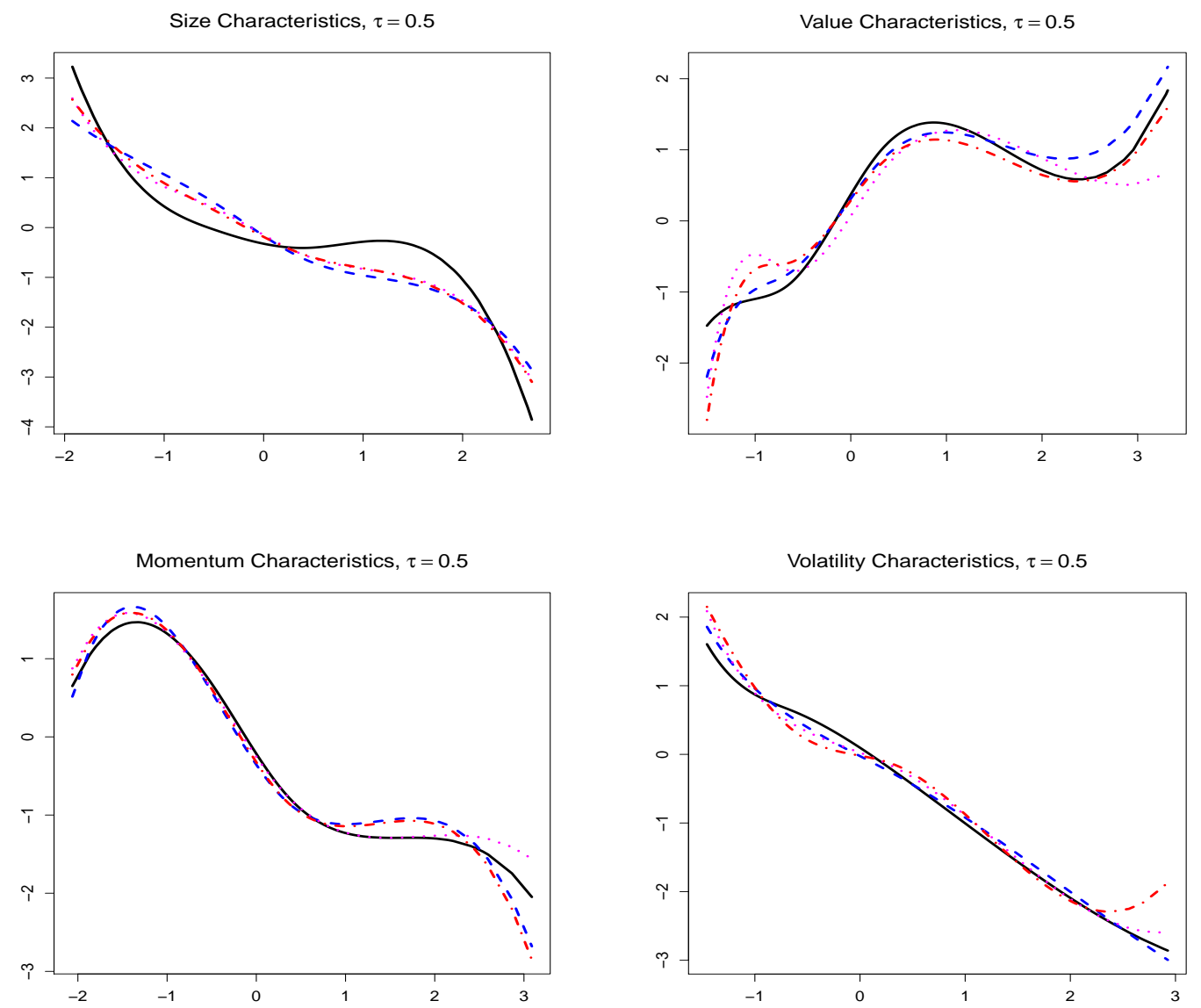

Figure 3: The plots of the estimated loading functions for the year of 2009 (dotted-dashed red lines), 2010 (dotted magenta lines), 2011 (dashed blue lines), and 2012 (solid black lines) at $\tau=0.8$.
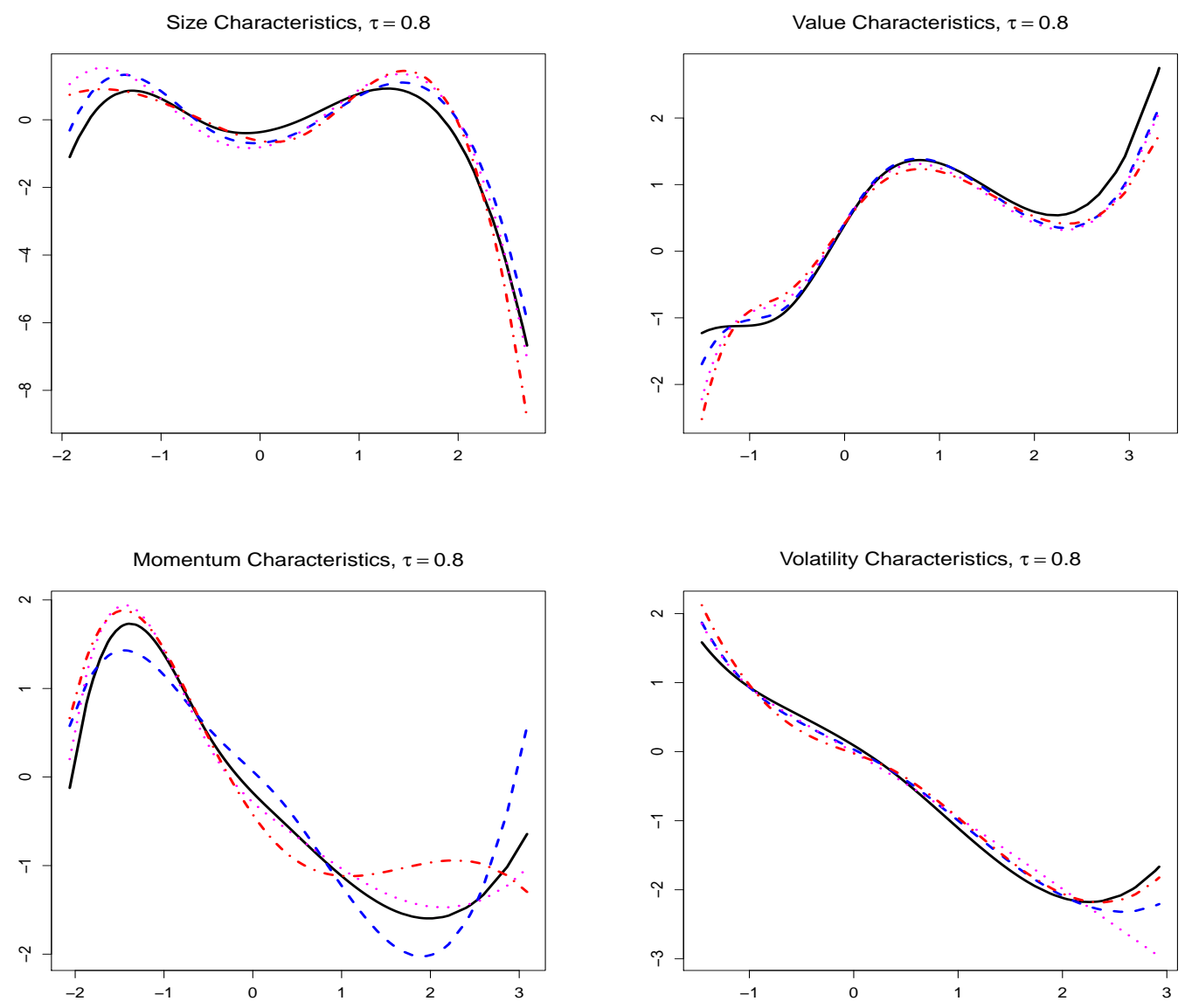
orderings of the data. Moreover, all five factors are statistically significant with the median p-value smaller than 0.05 .

\section{Conclusions and discussion}

We have taken for granted that the $J$ factors are present in the sense that

$$
\operatorname{plim}_{T \rightarrow \infty} \frac{1}{T} \sum_{t=1}^{T} f_{j t}^{0} \neq 0
$$

for $j=1, \ldots, J$. For the factors in our application this is quite a standard assumption, but in some cases one might wish to test this because if this condition fails, then the right hand side of (2.4) is close to zero and this equation cannot identify $g_{j}^{0}\left(x_{j}\right)$. We outline below a test of the hypothesis (7.1) based on the unstructured additive quantile regression model (2.3). A more limited objective is to test whether for a given time period $t, f_{j t}=0$.

We are interested in testing the hypothesis that

$$
H_{0_{A_{j}}}: \lim _{T \rightarrow \infty} \frac{1}{T} \sum_{t=1}^{T} h_{j t}\left(x_{j}\right)=0 \text { for all } x_{j},
$$

against the general alternative that $\lim _{T \rightarrow \infty} \frac{1}{T} \sum_{t=1}^{T} h_{j t}\left(x_{j}\right)=\mu_{j}\left(x_{j}\right)$ with $\int \mu_{j}\left(x_{j}\right)^{2} d P_{j}\left(x_{j}\right)>$ 0 . We also may be interested in a joint test $H_{0}=\cap_{j \in I_{J}} H_{0_{A_{j}}}$, where $I_{J}$ is a set of integers, which is a subset of $\{1,2, \ldots, J\}$. These are tests of the presence of a factor.

We let

$$
\widehat{\tau}_{j, N, T}=\frac{\int\left(\frac{1}{T} \sum_{t=1}^{T} \widehat{h}_{j t}\left(x_{j}\right)\right)^{2} d P_{j}\left(x_{j}\right)-a_{N, T}}{s_{N, T}}
$$

where $\widehat{h}_{j t}(\cdot)$ is an estimator of the additive component function $h_{j t}(\cdot)$ from the quantile additive model at time $t$, while $a_{N, T}$ and $s_{N, T}$ are constants to be determined. Under the null hypothesis (7.2) we may show that

$$
\widehat{\tau}_{j, n, T} \stackrel{\mathcal{D}}{\rightarrow} \mathcal{N}(0,1)
$$

while under the alternative we have $\widehat{\tau}_{j, n, T} \rightarrow \infty$ with probability approaching one. To ensure that $\widehat{\tau}_{j, n, T}$ has an asymptotic distribution, we may need a two-step estimator for the additive functions $h_{j t}(\cdot)$ as given in Horowitz and Mammen (2011) or Ma and Yang (2011). This interesting and challenging technical problem deserves further investigation, and it can be a good future research topic. 
Table 1: Factor return statistics at $\tau=0.5$ for the year of 2012 when the data are ordered according to the firm size.

\begin{tabular}{|c|c|c|c|c|c|c|}
\hline$(\kappa, b)$ & & Intercept & Size & Value & Momentum & Volatility \\
\hline \multirow{3}{*}{$(0.5,0.2)$} & Annualized volatility & 0.024 & 0.023 & 0.025 & 0.025 & 0.029 \\
\hline & $\%$ Periods significant & 92.43 & 65.34 & 62.95 & 64.54 & 74.10 \\
\hline & Overall p-value & $<0.001$ & 0.010 & 0.016 & 0.009 & 0.001 \\
\hline \multirow{3}{*}{$(0.5,0.4)$} & Annualized volatility & 0.020 & 0.020 & 0.022 & 0.022 & 0.026 \\
\hline & $\%$ Periods significant & 91.63 & 58.17 & 57.20 & 58.17 & 66.93 \\
\hline & Overall p-value & $<0.001$ & 0.020 & 0.019 & 0.020 & 0.010 \\
\hline \multirow{3}{*}{$(0.5,0.6)$} & Annualized volatility & 0.018 & 0.019 & 0.019 & 0.020 & 0.023 \\
\hline & $\%$ Periods significant & 90.84 & 55.78 & 56.40 & 55.38 & 66.93 \\
\hline & Overal p-value & $<0.001$ & 0.032 & 0.028 & 0.023 & 0.006 \\
\hline \multirow{3}{*}{$(1.0,0.2)$} & Annualized volatility & 0.026 & 0.026 & 0.027 & 0.027 & 0.032 \\
\hline & $\%$ Periods significant & 92.03 & 62.95 & 63.60 & 62.15 & 71.31 \\
\hline & Overall p-value & $<0.001$ & 0.014 & 0.019 & 0.011 & 0.002 \\
\hline \multirow{3}{*}{$(1.0,0.4)$} & Annualized volatility & 0.022 & 0.023 & 0.023 & 0.024 & 0.028 \\
\hline & $\%$ Periods significant & 90.44 & 55.20 & 56.40 & 55.98 & 65.74 \\
\hline & Overall p-value & $<0.001$ & 0.036 & 0.030 & 0.033 & 0.011 \\
\hline \multirow{3}{*}{$(1.0,0.6)$} & Annualized volatility & 0.019 & 0.022 & 0.021 & 0.021 & 0.025 \\
\hline & $\%$ Periods significant & 89.24 & 56.20 & 55.40 & 58.80 & 62.95 \\
\hline & Overall p-value & $<0.001$ & 0.032 & 0.032 & 0.026 & 0.016 \\
\hline \multirow{3}{*}{$(1.5,0.2)$} & Annualized volatility & 0.027 & 0.028 & 0.029 & 0.029 & 0.034 \\
\hline & $\%$ Periods significant & 92.03 & 59.76 & 55.38 & 61.75 & 70.12 \\
\hline & Overall p-value & $<0.001$ & 0.021 & 0.032 & 0.015 & 0.003 \\
\hline \multirow{3}{*}{$(1.5,0.4)$} & Annualized volatility & 0.023 & 0.025 & 0.025 & 0.026 & 0.031 \\
\hline & $\%$ Periods significant & 90.44 & 56.57 & 55.94 & 55.94 & 63.75 \\
\hline & Overall p-value & $<0.001$ & 0.030 & 0.030 & 0.036 & 0.014 \\
\hline \multirow{3}{*}{$(1.5,0.6)$} & Annualized volatility & 0.020 & 0.019 & 0.022 & 0.022 & 0.026 \\
\hline & $\%$ Periods significant & 88.44 & 58.14 & 56.80 & 56.00 & 61.75 \\
\hline & Overall p-value & $<0.001$ & 0.027 & 0.028 & 0.024 & 0.018 \\
\hline
\end{tabular}


Table 2: Factor return statistics at $\tau=0.5$ for the year of 2012 when the data are ordered according to the first principal component of the covariate matrix.

\begin{tabular}{|c|c|c|c|c|c|c|}
\hline$(\kappa, b)$ & & Intercept & Size & Value & Momentum & Volatility \\
\hline \multirow{3}{*}{$(0.5,0.2)$} & Annualized volatility & 0.023 & 0.027 & 0.025 & 0.025 & 0.027 \\
\hline & $\%$ Periods significant & 94.02 & 62.15 & 62.55 & 67.73 & 75.30 \\
\hline & Overall p-value & $<0.001$ & 0.023 & 0.018 & 0.011 & $<0.001$ \\
\hline \multirow{3}{*}{$(0.5,0.4)$} & Annualized volatility & 0.019 & 0.024 & 0.022 & 0.021 & 0.023 \\
\hline & $\%$ Periods significant & 92.43 & 57.60 & 54.20 & 58.96 & 70.92 \\
\hline & Overall p-value & $<0.001$ & 0.023 & 0.032 & 0.019 & 0.001 \\
\hline \multirow{3}{*}{$(0.5,0.6)$} & Annualized volatility & 0.016 & 0.021 & 0.020 & 0.019 & 0.020 \\
\hline & $\%$ Periods significant & 92.83 & 55.60 & 56.40 & 61.60 & 71.31 \\
\hline & Overal p-value & $<0.001$ & 0.028 & 0.028 & 0.018 & 0.004 \\
\hline \multirow{3}{*}{$(1.0,0.2)$} & Annualized volatility & 0.025 & 0.031 & 0.027 & 0.027 & 0.030 \\
\hline & $\%$ Periods significant & 93.23 & 56.18 & 58.96 & 66.14 & 73.71 \\
\hline & Overall p-value & $<0.001$ & 0.036 & 0.023 & 0.014 & 0.002 \\
\hline \multirow{3}{*}{$(1.0,0.4)$} & Annualized volatility & 0.020 & 0.026 & 0.024 & 0.024 & 0.025 \\
\hline & $\%$ Periods significant & 92.03 & 54.80 & 56.20 & 59.60 & 71.20 \\
\hline & Overall p-value & $<0.001$ & 0.030 & 0.030 & 0.019 & 0.002 \\
\hline \multirow{3}{*}{$(1.0,0.6)$} & Annualized volatility & 0.016 & 0.024 & 0.022 & 0.022 & 0.022 \\
\hline & $\%$ Periods significant & 92.80 & 56.20 & 55.40 & 56.80 & 68.80 \\
\hline & Overall p-value & $<0.001$ & 0.027 & 0.031 & 0.029 & 0.002 \\
\hline \multirow{3}{*}{$(1.5,0.2)$} & Annualized volatility & 0.027 & 0.030 & 0.029 & 0.028 & 0.032 \\
\hline & $\%$ Periods significant & 92.03 & 56.00 & 54.40 & 68.00 & 74.00 \\
\hline & Overall p-value & $<0.001$ & 0.033 & 0.032 & 0.013 & 0.002 \\
\hline \multirow{3}{*}{$(1.5,0.4)$} & Annualized volatility & 0.021 & 0.028 & 0.026 & 0.026 & 0.026 \\
\hline & $\%$ Periods significant & 92.03 & 56.60 & 55.90 & 55.20 & 68.00 \\
\hline & Overall p-value & $<0.001$ & 0.028 & 0.028 & 0.030 & 0.002 \\
\hline \multirow{3}{*}{$(1.5,0.6)$} & Annualized volatility & 0.018 & 0.025 & 0.024 & 0.023 & 0.024 \\
\hline & $\%$ Periods significant & 92.03 & 58.10 & 54.80 & 56.00 & 67.60 \\
\hline & Overall p-value & $<0.001$ & 0.027 & 0.030 & 0.029 & 0.003 \\
\hline
\end{tabular}




\section{Acknowledge}

Ma's research was partially supported by NSF grants DMS 1306972 and DMS 1712558. Gao's research was supported by the Australian Research Council Discovery Grants Program for its support under Grant numbers: DP150101012 \& DP170104421.

\section{Appendix}

We first introduce some notations which will be used throughout the Appendix. Let $\lambda_{\max }(\mathbf{A})$ and $\lambda_{\min }(\mathbf{A})$ denote the largest and smallest eigenvalues of a symmetric matrix $\mathbf{A}$, respectively. For an $m \times n$ real matrix $\mathbf{A}$, we denote $\|\mathbf{A}\|_{\infty}=\max _{1 \leq i \leq m} \sum_{j=1}^{n}\left|A_{i j}\right|$. For any vector $\mathbf{a}=\left(a_{1}, \ldots, a_{n}\right)^{\top} \in \mathbb{R}^{n}$, denote $\|\mathbf{a}\|_{\infty}=\max _{1 \leq i \leq n}\left|a_{i}\right|$. We first study the asymptotic properties of the initial estimators $\widehat{g}_{j}^{[0]}\left(x_{j}\right)$ of $g_{j}^{0}\left(x_{j}\right)$. The following proposition gives the convergence rate of $\widehat{g}_{j}^{[0]}\left(x_{j}\right)$ that will be used in the proofs of Theorems 1 and 2 .

Proposition 1. Let Conditions (C1)-(C4) hold. If, in addition, $K_{N}^{4} N^{-1}=o(1), K_{N}^{-r+2}(\log T)=$ $o(1)$ and $K_{N}^{-1}(\log N T)(\log N)^{4}=o(1)$, then for every $1 \leq j \leq J$,

$$
\begin{gathered}
\sup _{x_{j} \in[a, b]}\left|\widehat{g}_{j}^{[0]}\left(x_{j}\right)-g_{j}^{0}\left(x_{j}\right)\right|=O_{p}\left(K_{N} / \sqrt{N T}+K_{N}^{2} N^{-3 / 4} \sqrt{\log N T}+K_{N}^{-r}\right)+o_{p}\left(N^{-1 / 2}\right), \\
{\left[\int\left\{\widehat{g}_{j}^{[0]}\left(x_{j}\right)-g_{j}^{0}\left(x_{j}\right)\right\}^{2} d x_{j}\right]^{1 / 2}=O_{p}\left(\sqrt{K_{N} /(N T)}+K_{N}^{3 / 2} N^{-3 / 4} \sqrt{\log N T}+K_{N}^{-r}\right)+o_{p}\left(N^{-1 / 2}\right) .}
\end{gathered}
$$

\subsection{Proof of Proposition 1}

According to the result on page 149 of de Boor (2001), for $h_{j t}^{0}$ satisfying the smoothness condition given in (C2), there exists $\boldsymbol{\theta}_{j t}^{0} \in \mathbb{R}^{K_{n}}$ such that $h_{j t}^{0}\left(x_{j}\right)=\widetilde{h}_{j t}^{0}\left(x_{j}\right)+b_{j t}\left(x_{j}\right)$

$$
\widetilde{h}_{j t}^{0}\left(x_{j}\right)=B_{j}\left(x_{j}\right)^{\top} \boldsymbol{\theta}_{j t}^{0} \text { and } \sup _{j, t} \sup _{x_{j} \in[a, b]}\left|b_{j t}\left(x_{j}\right)\right|=O\left(K_{N}^{-r}\right) .
$$

Denote $\widetilde{h}_{t}^{0}(x)=\left\{\widetilde{h}_{j t}^{0}\left(x_{j}\right), 1 \leq j \leq J\right\}^{\top}$, and

$$
b_{t}(x)=\sum_{j=1}^{J} h_{j t}^{0}\left(x_{j}\right)-B(x)^{\top} \boldsymbol{\theta}_{t}^{0},
$$

where $B(x)=\left\{B_{1}\left(x_{1}\right)^{\top}, \ldots, B_{J}\left(x_{J}\right)^{\top}\right\}^{\top}$ and $\boldsymbol{\theta}_{t}^{0}=\left(\boldsymbol{\theta}_{1 t}^{0 \top}, \ldots, \boldsymbol{\theta}_{J t}^{0 \top}\right)^{\top}$. Then by (A.1), we have

$$
\sup _{x \in[a, b]^{J}}\left|b_{t}(x)\right|=O\left(K_{N}^{-r}\right) .
$$

Then $\mathbb{B}(x)\left(\widetilde{h}_{u t}, \widetilde{\boldsymbol{\theta}}_{t}^{\top}\right)^{\boldsymbol{\top}}=\left(\widetilde{h}_{u t}, \widetilde{h}_{t}(x)^{\top}\right)^{\top}$ and $\mathbb{B}(x)\left(h_{u t}^{0}, \boldsymbol{\theta}_{t}^{0 \top}\right)^{\top}=\left(h_{u t}^{0}, \widetilde{h}_{t}^{0}(x)^{\top}\right)^{\top}$, where

$$
\mathbb{B}(x)=\left[\operatorname{diag}\left\{1, B_{1}\left(x_{1}\right)^{\top}, \ldots, B_{J}\left(x_{J}\right)^{\top}\right\}\right]_{(1+J) \times\left(1+J K_{N}\right)},
$$

$\widetilde{h}_{t}(x)=\left\{\widetilde{h}_{j t}\left(x_{j}\right), 1 \leq j \leq J\right\}^{\top}$, and $\widetilde{h}_{j t}(\cdot)$ are the estimators given in Section 3.2. We first give the Bernstein inequality for a $\phi$-mixing sequence, which is used through our proof. 
Lemma 1. Let $\left\{\xi_{i}\right\}$ be a sequence of centered real-valued random variables. Let $S_{n}=\sum_{i=1}^{n} \xi_{i}$. Suppose the sequence has the $\phi$-mixing coefficient satisfying $\phi(k) \leq \exp (-2 c k)$ for some $c>0$ and $\sup _{i \geq 1}\left|\xi_{i}\right| \leq M$. Then there is a positive constant $C_{1}$ depending only on $c$ such that for all $n \geq 2$

$$
P\left(\left|S_{n}\right| \geq \varepsilon\right) \leq \exp \left(-\frac{C_{1} \varepsilon^{2}}{v^{2} n+M^{2}+\varepsilon M(\log n)^{2}}\right),
$$

where $v^{2}=\sup _{i>0}\left(\operatorname{var}\left(\xi_{i}\right)+2 \sum_{j>i}\left|\operatorname{cov}\left(\xi_{i}, \xi_{j}\right)\right|\right)$.

Proof. The result of Lemma 1 is given in Theorem 2 on page 275 of Merlevéde, Peligrad and Rio (2009) when the sequence $\left\{\xi_{i}\right\}$ has the $\alpha$-mixing coefficient satisfying $\alpha(k) \leq \exp (-2 c k)$ for some $c>0$. Thus, this result also holds for the sequence having the $\phi$-mixing coefficient satisfying $\phi(k) \leq \exp (-2 c k)$, since $\alpha(k) \leq \phi(k) \leq \exp (-2 c k)$.

Denote $B\left(X_{i}\right)=\left\{B_{1}\left(X_{1 i}\right)^{\top}, \ldots, B_{J}\left(X_{J i}\right)^{\top}\right\}^{\top}$ and $Z_{i}=\left[\left\{1, B\left(X_{i}\right)^{\top}\right\}^{\top}\right]_{\left(1+J K_{N}\right) \times 1}$. Denote $\boldsymbol{\vartheta}_{t}=\left(h_{u t}, \boldsymbol{\theta}_{t}^{\top}\right)^{\top}$ and $\boldsymbol{\vartheta}_{t}^{0}=\left(h_{u t}^{0}, \boldsymbol{\theta}_{t}^{0 \top}\right)^{\top}$. Define

$$
\begin{aligned}
& G_{t N, i}\left(\boldsymbol{\vartheta}_{t}\right)=\left[\tau-I\left\{\varepsilon_{i t} \leq Z_{i}^{\top}\left(\boldsymbol{\vartheta}_{t}-\boldsymbol{\vartheta}_{t}^{0}\right)-b_{t}\left(X_{i}\right)\right\}\right] Z_{i}, \\
& G_{t N, i}^{*}\left(\boldsymbol{\vartheta}_{t}\right)=\left[\tau-F_{i}\left[\left\{Z_{i}^{\top}\left(\boldsymbol{\vartheta}_{t}-\boldsymbol{\vartheta}_{t}^{0}\right)-b_{t}\left(X_{i}\right)\right\} \mid X_{i}, f_{t}\right]\right] Z_{i},
\end{aligned}
$$

where $F_{i}\left(\varepsilon \mid X_{i}, f_{t}\right)=P\left(\varepsilon_{i t} \leq \varepsilon \mid X_{i}, f_{t}\right)$, and $\widetilde{G}_{t N, i}\left(\boldsymbol{\vartheta}_{t}\right)=G_{t N, i}\left(\boldsymbol{\vartheta}_{t}\right)-G_{t N, i}^{*}\left(\boldsymbol{\vartheta}_{t}\right)$. Let $d(N)=$ $\left(1+J K_{N}\right)$.

Let $\Psi_{N t}=N^{-1} \sum_{i=1}^{N} p_{i}\left(0 \mid X_{i}, f_{t}\right) Z_{i} Z_{i}^{\top}$. By the same reasoning as the proofs for (ii) of Lemma A.7 in Ma and Yang (2011), we have with probability approaching 1 , as $N \rightarrow \infty$, there exist constants $0<C_{1} \leq C_{2}<\infty$ such that

$$
C_{1} \leq \lambda_{\min }\left(\Psi_{N t}\right) \leq \lambda_{\max }\left(\Psi_{N t}\right) \leq C_{2}
$$

uniformly in $t=1, \ldots, T$.

Next lemma presents the Bahadur representation for $\widetilde{\boldsymbol{\vartheta}}_{t}=\left(\widetilde{h}_{u t}, \widetilde{\boldsymbol{\theta}}_{t}^{\top}\right)^{\top}$ using the results in Lemmas S.1.S.3 given in the Supplemental Materials.

Lemma 2. Under Conditions (C1)-(C3), and $K_{N}^{3} N^{-1}=o(1), K_{N}^{2} N^{-1}(\log N T)^{2}(\log N)^{8}=$ $o(1)$ and $K_{N}^{-r+1}(\log T)=o(1)$,

$$
\widetilde{\boldsymbol{\vartheta}}_{t}-\boldsymbol{\vartheta}_{t}^{0}=D_{N t, 1}+D_{N t, 2}+R_{N t}
$$

where

$$
\begin{gathered}
D_{N t, 1}=\Psi_{N t}^{-1}\left[N^{-1} \sum_{i=1}^{N} Z_{i}\left(\tau-I\left(\varepsilon_{i t}<0\right)\right)\right] \\
D_{N t, 2}=\Psi_{N t}^{-1}\left[N^{-1} \sum_{i=1}^{N} Z_{i}\left\{p_{i}\left(0 \mid X_{i}, f_{t}\right) \sum_{j=1}^{J} b_{j t}\left(X_{j i}\right)\right\}\right],
\end{gathered}
$$

uniformly in $t$, and the remaining term $R_{N t}$ satisfies

$$
\begin{aligned}
\sup _{1 \leq t \leq T}\left\|R_{N t}\right\| & =O_{p}\left(K_{N}^{3 / 2} N^{-1}+K_{N}^{3 / 2} N^{-3 / 4} \sqrt{\log N T}+K_{N}^{1 / 2-2 r}+N^{-1 / 2} K_{N}^{-r / 2+1 / 2} \sqrt{\log K_{N} T}\right) \\
& =O_{p}\left(K_{N}^{3 / 2} N^{-3 / 4} \sqrt{\log N T}+K_{N}^{1 / 2-2 r}\right)+o_{p}\left(N^{-1 / 2}\right) .
\end{aligned}
$$


Proof. By Lemma S.3 in the Supplemental Materials, we have

$$
\widetilde{\boldsymbol{\vartheta}}_{t}-\boldsymbol{\vartheta}_{t}^{0}=N^{-1} \Psi_{N t}^{-1} \sum_{i=1}^{N} p_{i}\left(0 \mid X_{i}, f_{t}\right) Z_{i} b_{t}\left(X_{i}\right)-\Psi_{N t}^{-1} G_{t N, i}^{*}\left(\widetilde{\vartheta}_{t}\right)+R_{N t}^{*}
$$

Moreover,

$$
\Psi_{N t}^{-1} G_{t N, i}^{*}\left(\widetilde{\boldsymbol{\vartheta}}_{t}\right)=\Psi_{N t}^{-1} G_{t N, i}\left(\widetilde{\boldsymbol{\vartheta}}_{t}\right)-\Psi_{N t}^{-1} \widetilde{G}_{t N, i}\left(\boldsymbol{\vartheta}_{t}^{0}\right)-\Psi_{N t}^{-1}\left[\widetilde{G}_{t N, i}\left(\widetilde{\boldsymbol{\vartheta}}_{t}\right)-\widetilde{G}_{t N, i}\left(\boldsymbol{\vartheta}_{t}^{0}\right)\right]
$$

Thus,

$$
\widetilde{\boldsymbol{\vartheta}}_{t}-\boldsymbol{\vartheta}_{t}^{0}=\Psi_{N t}^{-1} N^{-1} \sum_{i=1}^{N} \widetilde{G}_{t N, i}\left(\boldsymbol{\vartheta}_{t}^{0}\right)+\Psi_{N t}^{-1} N^{-1} \sum_{i=1}^{N} p_{i}\left(0 \mid X_{i}, f_{t}\right) Z_{i} b_{t}\left(X_{i}\right)+R_{N t}^{* *},
$$

where

$$
R_{N t}^{* *}=-\Psi_{N t}^{-1} N^{-1} \sum_{i=1}^{N} G_{t N, i}\left(\widetilde{\vartheta}_{t}\right)+\Psi_{N t}^{-1} N^{-1} \sum_{i=1}^{N}\left[\widetilde{G}_{t N, i}\left(\widetilde{\boldsymbol{\vartheta}}_{t}\right)-\widetilde{G}_{t N, i}\left(\boldsymbol{\vartheta}_{t}^{0}\right)\right]+R_{N t}^{*} .
$$

By Lemmas $\mathbf{S . 1}$ and $\mathbf{S . 2}$ in the Supplemental Materials and (A.3), we have

$$
\begin{aligned}
\sup _{1 \leq t \leq T}\left\|R_{N t}^{* *}\right\| & \leq \sup _{1 \leq t \leq T}\left\|\Psi_{N t}^{-1}\right\| \sup _{1 \leq t \leq T}\left\|N^{-1} \sum_{i=1}^{N} G_{t N, i}\left(\widetilde{\vartheta}_{t}\right)\right\| \\
& +\sup _{1 \leq t \leq T}\left\|\Psi_{N t}^{-1}\right\| \sup _{1 \leq t \leq T}\left\|N^{-1} \sum_{i=1}^{N}\left[\widetilde{G}_{t N, i}\left(\widetilde{\vartheta}_{t}\right)-\widetilde{G}_{t N, i}\left(\boldsymbol{\vartheta}_{t}^{0}\right)\right]\right\|+\sup _{1 \leq t \leq T}\left\|R_{N t}^{*}\right\| \\
& =O_{p}\left(K_{N}^{3 / 2} N^{-1}+\left(K_{N}^{2} N\right)^{-3 / 4} \sqrt{\log N T}+K_{N}^{1 / 2-2 r}\right) .
\end{aligned}
$$

Define $\bar{G}_{t N, i \ell}\left(\boldsymbol{\vartheta}_{t}^{0}\right)=\left\{\tau-I\left(\varepsilon_{i t} \leq 0\right)\right\} Z_{i, \ell}$ and $\bar{G}_{t N, i}\left(\boldsymbol{\vartheta}_{t}^{0}\right)=\left\{\bar{G}_{t N, i \ell}\left(\boldsymbol{\vartheta}_{t}^{0}\right), 1 \leq \ell \leq d(N)\right\}$. Then $E\left\{\widetilde{G}_{t N, i \ell}\left(\boldsymbol{\vartheta}_{t}^{0}\right)-\bar{G}_{t N, i \ell}\left(\boldsymbol{\vartheta}_{t}^{0}\right)\right\}=0$. Moreover,

$$
E\left\{\widetilde{G}_{t N, i \ell}\left(\boldsymbol{\vartheta}_{t}^{0}\right)-\bar{G}_{t N, i \ell}\left(\boldsymbol{\vartheta}_{t}^{0}\right)\right\}^{2} \leq E\left[I\left\{\varepsilon_{i t} \leq-b_{t}\left(X_{i}\right)\right\}-I\left\{\varepsilon_{i t} \leq 0\right\} Z_{i, \ell}\right]^{2} \leq C K_{N}^{-r}
$$

for some constant $0<C<\infty$, and by Condition (C1), we have

$$
\begin{aligned}
& E\left\{\widetilde{G}_{t N, i \ell}\left(\boldsymbol{\vartheta}_{t}^{0}\right)-\bar{G}_{t N, i \ell}\left(\boldsymbol{\vartheta}_{t}^{0}\right)\right\}\left\{\widetilde{G}_{t N, i^{\prime} \ell}\left(\boldsymbol{\vartheta}_{t}^{0}\right)-\bar{G}_{t N, i^{\prime} \ell}\left(\boldsymbol{\vartheta}_{t}^{0}\right)\right\} \\
& \leq 2 \times 4^{2}\left\{\phi\left(\left|i^{\prime}-i\right|\right)\right\}^{1 / 2}\left[E\left\{\widetilde{G}_{t N, i \ell}\left(\boldsymbol{\vartheta}_{t}^{0}\right)-\bar{G}_{t N, i \ell}\left(\boldsymbol{\vartheta}_{t}^{0}\right)\right\}^{2} E\left\{\widetilde{G}_{t N, i^{\prime} \ell}\left(\boldsymbol{\vartheta}_{t}^{0}\right)-\bar{G}_{t N, i^{\prime} \ell}\left(\boldsymbol{\vartheta}_{t}^{0}\right)\right\}^{2}\right]^{1 / 2} \\
& \leq C^{\prime} K_{1} e^{-\lambda_{1}\left|i^{\prime}-i\right| / 2} K_{N}^{-r} .
\end{aligned}
$$

Hence, by the above results, we have

$$
\begin{aligned}
& E\left[N^{-1} \sum_{i=1}^{N}\left\{\widetilde{G}_{t N, i \ell}\left(\boldsymbol{\vartheta}_{t}^{0}\right)-\bar{G}_{t N, i \ell}\left(\boldsymbol{\vartheta}_{t}^{0}\right)\right\}\right]^{2} \\
& \leq N^{-1} C K_{N}^{-r}+N^{-2} \sum_{i \neq i^{\prime}} C^{\prime} K_{1} e^{-\lambda_{1}\left|i^{\prime}-i\right|} K_{N}^{-r} \\
& \leq C N^{-1} K_{N}^{-r}+C^{\prime} K_{1} N^{-2} N\left(1-e^{-\lambda_{1} / 2}\right)^{-1} K_{N}^{-r} \leq C^{\prime \prime} N^{-1} K_{N}^{-r},
\end{aligned}
$$

for some constant $0<C^{\prime \prime}<\infty$. Thus

$$
\begin{aligned}
E\left\|N^{-1} \sum_{i=1}^{N}\left\{\widetilde{G}_{t N, i}\left(\boldsymbol{\vartheta}_{t}^{0}\right)-\bar{G}_{t N, i}\left(\boldsymbol{\vartheta}_{t}^{0}\right)\right\}\right\|^{2} & =\sum_{\ell=1}^{d(N)} E\left[N^{-1} \sum_{i=1}^{N}\left\{\widetilde{G}_{t N, i \ell}\left(\boldsymbol{\vartheta}_{t}^{0}\right)-\bar{G}_{t N, i \ell}\left(\boldsymbol{\vartheta}_{t}^{0}\right)\right\}\right]^{2} \\
& \leq C^{\prime \prime}\left(1+J K_{N}\right) N^{-1} K_{N}^{-r} .
\end{aligned}
$$


Therefore, by the Bernstein's inequality in Lemma 1 and the union bound of probability, following the same procedure as the proof for Lemma S.1 given in the Supplemental Materials, we have

$$
\sup _{1 \leq t \leq T}\left\|N^{-1} \sum_{i=1}^{N}\left\{\widetilde{G}_{t N, i}\left(\boldsymbol{\vartheta}_{t}^{0}\right)-\bar{G}_{t N, i}\left(\boldsymbol{\vartheta}_{t}^{0}\right)\right\}\right\|=O_{p}\left(N^{-1 / 2} K_{N}^{-r / 2+1 / 2} \sqrt{\log K_{N} T}\right) .
$$

Therefore, by (A.6), (A.7) and (A.8), we have $\widetilde{\vartheta}_{t}-\boldsymbol{\vartheta}_{t}^{0}=D_{N t, 1}+D_{N t, 2}+R_{N t}$, where

$$
\sup _{1 \leq t \leq T}\left\|R_{N t}\right\|=O_{p}\left(K_{N}^{3 / 2} N^{-1}+\left(K_{N}^{2} N\right)^{-3 / 4} \sqrt{\log N T}+K_{N}^{1 / 2-2 r}+N^{-1 / 2} K_{N}^{-r / 2+1 / 2} \sqrt{\log K_{N} T}\right) .
$$

Proof of Proposition 1. Let $1_{l}$ be the $(J+1) \times 1$ vector with the $l^{\text {th }}$ element as " 1 " and other elements as "0". By (A.4) in Lemma 2, we have

$$
\begin{gathered}
\tilde{h}_{j t}\left(x_{j}\right)-\widetilde{h}_{j t}^{0}\left(x_{j}\right)=1_{j+1}^{\top} \mathbb{B}(x)\left(D_{N t, 1}+D_{N t, 2}\right)+1_{j+1}^{\top} \mathbb{B}(x) R_{N t} \\
\sup _{1 \leq t \leq T}\left\{N^{-1} \sum_{i=1}^{N}\left(1_{j+1}^{\top} \mathbb{B}\left(X_{i}\right) R_{N t}\right)^{2}\right\}^{1 / 2} \\
\leq \sup _{1 \leq t \leq T}\left\|R_{N t}\right\|\left[\lambda_{\max }\left\{N^{-1} \sum_{i=1}^{N} B_{j}\left(X_{j i}\right) B_{j}\left(X_{j i}\right)^{\top}\right\}\right]^{1 / 2} \\
=O_{p}\left(K_{N}^{3 / 2} N^{-3 / 4} \sqrt{\log N T}+K_{N}^{1 / 2-2 r}\right)+o_{p}\left(N^{-1 / 2}\right),
\end{gathered}
$$

and

$$
\begin{aligned}
& \sup _{1 \leq t \leq T} \sup _{x \in[a, b]^{J}}\left|1_{j+1}^{\top} \mathbb{B}(x) R_{N t}\right| \\
& \leq \sup _{x \in[a, b]^{J}}\left\|\mathbb{B}(x)^{\top} 1_{j+1}|| \sup _{1 \leq t \leq T}|| R_{N t}\right\| \\
& =O\left(K_{N}^{1 / 2}\right) O_{p}\left(K_{N}^{3 / 2} N^{-1}+K_{N}^{3 / 2} N^{-3 / 4} \sqrt{\log N T}+K_{N}^{1 / 2-2 r}+N^{-1 / 2} K_{N}^{-r / 2+1 / 2} \sqrt{\log K_{N} T}\right) \\
& =O_{p}\left(K_{N}^{2} N^{-3 / 4} \sqrt{\log N T}+K_{N}^{1-2 r}\right)+o_{p}\left(N^{-1 / 2}\right),
\end{aligned}
$$

by the assumption that $K_{N}^{4} N^{-1}=o(1), K_{N}^{-r+2}(\log T)=o(1)$ and $r>2$. Since $h_{j t}^{0}\left(x_{j}\right)=$ $\widetilde{h}_{j t}^{0}\left(x_{j}\right)+b_{j t}\left(x_{j}\right)$, then we have

$$
\widetilde{h}_{j t}\left(x_{j}\right)-h_{j t}^{0}\left(x_{j}\right)=1_{j+1}^{\top} \mathbb{B}(x)\left(D_{N t, 1}+D_{N t, 2}\right)-b_{j t}\left(x_{j}\right)+1_{j+1}^{\top} \mathbb{B}(x) R_{N t} .
$$

Also by (A.1), we have $\sup _{1 \leq t \leq T} \sup _{x \in[a, b]^{J}}\left|1_{j+1}^{\top} \mathbb{B}(x) D_{N t, 2}\right|=O_{p}\left(K_{N}^{-r}\right)$. Then $\widetilde{h}_{j t}\left(x_{j}\right)-$ $h_{j t}^{0}\left(x_{j}\right)$ can be written as

$$
\widetilde{h}_{j t}\left(x_{j}\right)-h_{j t}^{0}\left(x_{j}\right)=1_{j+1}^{\top} \mathbb{B}(x) D_{N t, 1}+\eta_{N, j t}\left(x_{j}\right),
$$

where the remaining term $\eta_{N, j t}\left(x_{j}\right)$ satisfies

$$
\sup _{1 \leq t \leq T}\left[N^{-1} \sum_{i=1}^{N}\left\{\eta_{N, j t}\left(X_{j i}\right)\right\}^{2}\right]^{1 / 2}=O_{p}\left(K_{N}^{-r}\right)+O_{p}\left(K_{N}^{3 / 2} N^{-3 / 4} \sqrt{\log N T}\right)+o_{p}\left(N^{-1 / 2}\right)
$$




$$
\begin{aligned}
& \sup _{1 \leq t \leq T}\left\{\int \eta_{N, j t}\left(x_{j}\right)^{2} d x_{j}\right\}^{1 / 2}=O_{p}\left(K_{N}^{-r}\right)+O_{p}\left(K_{N}^{3 / 2} N^{-3 / 4} \sqrt{\log N T}\right)+o_{p}\left(N^{-1 / 2}\right), \\
& \sup _{1 \leq t \leq T} \sup _{x_{j} \in[a, b]}\left|\eta_{N, j t}\left(x_{j}\right)\right|=O_{p}\left(K_{N}^{-r}\right)+O_{p}\left(K_{N}^{2} N^{-3 / 4} \sqrt{\log N T}\right)+o_{p}\left(N^{-1 / 2}\right) .
\end{aligned}
$$

Moreover, by Berntein's inequality and following the same procedure as the proof for Lemma S.1. we have $\sup _{1 \leq t \leq T}|| D_{N t, 1} \|=O_{p}\left(\sqrt{K_{N} / N} \sqrt{\log K_{N} T}\right)$. Hence,

$$
\begin{array}{r}
\sup _{1 \leq t \leq T} \sup _{x \in[a, b]^{J}}\left|1_{j+1}^{\top} \mathbb{B}(x) D_{N t, 1}\right|=O_{p}\left(\sqrt{\log K_{N} T} K_{N} / \sqrt{N}\right), \\
\sup _{1 \leq t \leq T}\left\{N^{-1} \sum_{i=1}^{N}\left(1_{j+1}^{\top} \mathbb{B}\left(X_{i}\right) D_{N t, 1}\right)^{2}\right\}^{1 / 2}=O_{p}\left(\sqrt{\log K_{N} T} \sqrt{K_{N} / N}\right) .
\end{array}
$$

Therefore, by (A.9), (A.10), (A.11) and (A.12), we have

$$
\begin{aligned}
& \sup _{1 \leq t \leq T} N^{-1} \sum_{i=1}^{N}\left\{\widetilde{h}_{j t}\left(X_{j i}\right)-h_{j t}^{0}\left(X_{j i}\right)\right\}^{2}=O_{p}\left(\left(\log K_{N} T\right) K_{N} / N+N^{-2 r}\right), \\
& \sup _{1 \leq t \leq T} \sup _{x_{j} \in[a, b]}\left|\widetilde{h}_{j t}\left(x_{j}\right)-h_{j t}^{0}\left(x_{j}\right)\right|=O_{p}\left(\sqrt{\log K_{N} T} K_{N} N^{-1 / 2}+K_{N}^{-r}\right) .
\end{aligned}
$$

Moreover, by Conditions (C3) and (C4), we have with probability approaching 1 , as $N \rightarrow \infty$,

$$
c_{h} \leq N^{-1} \sum_{i=1}^{N}\left(T^{-1} \sum_{t=1}^{T} h_{j t}^{0}\left(X_{j i}\right)\right)^{2} \leq C_{h}, c_{h} \leq N^{-1} \sum_{i=1}^{N}\left(T^{-1} \sum_{t=1}^{T} \widetilde{h}_{j t}\left(X_{j i}\right)\right)^{2} \leq C_{h} .
$$

Hence, this result together with (A.9) leads to that with probability approaching 1, as $N \rightarrow \infty$,

$$
\begin{aligned}
& \left|1 / \sqrt{N^{-1} \sum_{i=1}^{N}\left(T^{-1} \sum_{t=1}^{T} \widetilde{h}_{j t}\left(X_{j i}\right)\right)^{2}}-1 / \sqrt{N^{-1} \sum_{i=1}^{N}\left(T^{-1} \sum_{t=1}^{T} h_{j t}^{0}\left(X_{j i}\right)\right)^{2}}\right| \\
& =\left|M_{N T} N^{-1} \sum_{i=1}^{N} T^{-1} \sum_{t=1}^{T}\left\{\widetilde{h}_{j t}\left(X_{j i}\right)-h_{j t}^{0}\left(X_{j i}\right)\right\} T^{-1} \sum_{t=1}^{T}\left\{\widetilde{h}_{j t}\left(X_{j i}\right)+h_{j t}^{0}\left(X_{j i}\right)\right\}\right| \\
& =\mid 2 M_{N T} N^{-1} \sum_{i=1}^{N} T^{-1} \sum_{t=1}^{T}\left\{\widetilde{h}_{j t}\left(X_{j i}\right)-h_{j t}^{0}\left(X_{j i}\right)\right\}\left\{T^{-1} \sum_{t=1}^{T} h_{j t}^{0}\left(X_{j i}\right)\right\} \\
& +M_{N T} N^{-1} \sum_{i=1}^{N} T^{-1} \sum_{t=1}^{T}\left\{\widetilde{h}_{j t}\left(X_{j i}\right)-h_{j t}^{0}\left(X_{j i}\right)\right\}^{2} \mid \\
& \leq\left|2 M_{N T} N^{-1} \sum_{i=1}^{N} T^{-1} \sum_{t=1}^{T}\left[1_{j+1}^{\top} \mathbb{B}\left(X_{i}\right) D_{N t, 1}\left\{T^{-1} \sum_{t=1}^{T} h_{j t}^{0}\left(X_{j i}\right)\right\}+\varrho_{i t}\right]\right| \\
& +2 M_{N T} N^{-1} \sum_{i=1}^{N} T^{-1} \sum_{t=1}^{T}\left\{1_{j+1}^{\top} \mathbb{B}\left(X_{i}\right) D_{N t, 1}\right\}^{2}+\eta_{N, j t}\left(X_{j i}\right) \\
& +2 M_{N T} N^{-1} \sum_{i=1}^{N} T^{-1} \sum_{t=1}^{T}\left\{\eta_{N, j t}\left(X_{j i}\right)\right\}^{2}
\end{aligned}
$$

for $M_{N T}$ satisfying $M_{N T} \in\left(c^{\prime}, C^{\prime}\right)$ for some constants $0<c^{\prime}<C^{\prime}<\infty$, where $\varrho_{i t}=$ $\eta_{N, j t}\left(X_{j i}\right)\left\{T^{-1} \sum_{t=1}^{T} h_{j t}^{0}\left(X_{j i}\right)\right\}$. Moreover by (A.10), there exists a constant $C^{*} \in(0, \infty)$ such that

$$
\begin{aligned}
\left|N^{-1} \sum_{i=1}^{N} T^{-1} \sum_{t=1}^{T} \varrho_{i t}\right| & \leq C^{*} \sup _{1 \leq t \leq T} N^{-1} \sum_{i=1}^{N}\left|\eta_{N, j t}\left(X_{j i}\right)\right| \\
& \leq C^{*} \sup _{1 \leq t \leq T}\left[N^{-1} \sum_{i=1}^{N}\left\{\eta_{N, j t}\left(X_{j i}\right)\right\}^{2}\right]^{1 / 2} \\
& =O_{p}\left(K_{N}^{-r}\right)+O_{p}\left(K_{N}^{3 / 2} N^{-3 / 4} \sqrt{\log N T}\right)+o_{p}\left(N^{-1 / 2}\right)
\end{aligned}
$$


and

$$
N^{-1} \sum_{i=1}^{N} T^{-1} \sum_{t=1}^{T}\left\{\eta_{N, j t}\left(X_{j i}\right)\right\}^{2}=O_{p}\left(K_{N}^{-2 r}\right)+O_{p}\left(K_{N}^{3} N^{-3 / 2} \log (N T)\right)+o_{p}\left(N^{-1}\right) .
$$

Define $\psi_{i t}=\left\{\psi_{i t, \ell}\right\}_{\ell=1}^{d(N)}=\Psi_{N t}^{-1} Z_{i}\left(\tau-I\left(\varepsilon_{i t}<0\right)\right)$. Then $E\left(\psi_{i t, \ell}\right)=0$. Moreover, $E\left\|\psi_{i t}\right\|^{2} \leq$ $c_{1} K_{N}$ for some constant $0<c_{1}<\infty$, and by Condition (C1), we have

$$
\begin{aligned}
\left|E\left(\psi_{i t}^{\top} \psi_{j s}\right)\right| & \leq 2\left\{\phi\left(\sqrt{|i-j|^{2}+|t-s|^{2}}\right)\right\}^{1 / 2} \sum_{\ell=1}^{d(N)}\left\{E\left(\psi_{i t, \ell}\right)^{2} E\left(\psi_{j s, \ell}\right)^{2}\right\}^{1 / 2} \\
& \leq\left\{\phi\left(\sqrt{|i-j|^{2}+|t-s|^{2}}\right)\right\}^{1 / 2}\left(\left.E|| \psi_{i t}\right|^{2}+E \|\left.\psi_{j s}\right|^{2}\right) \\
& \leq 2 c_{1} K_{N}\left\{\phi\left(\sqrt{|i-j|^{2}+|t-s|^{2}}\right)\right\}^{1 / 2} .
\end{aligned}
$$

Hence by Condition (C1), we have

$$
\begin{aligned}
& E\left\|(N T)^{-1} \sum_{t=1}^{T} \sum_{i=1}^{N} \psi_{i t}\right\|^{2} \\
& =(N T)^{-2} \sum_{t, t^{\prime}} \sum_{i, i^{\prime}} E\left(\psi_{i t}^{\top} \psi_{i^{\prime} t^{\prime}}\right) \leq 2 c_{1} K_{N}(N T)^{-2} \sum_{t, t^{\prime}} \sum_{i, i^{\prime}}\left\{\phi\left(\sqrt{|i-j|^{2}+|t-s|^{2}}\right)\right\}^{1 / 2} \\
& \leq 2 c_{1} K_{1} K_{N}(N T)^{-2} \sum_{t, t^{\prime}} \sum_{i, i^{\prime}} e^{-\lambda_{1} \sqrt{\left|i-i^{\prime}\right|^{2}+\left|t-t^{\prime}\right|^{2}} / 2} \\
& \leq 2 c_{1}(N T)^{-2} K_{1} K_{N} \sum_{t, t^{\prime}} \sum_{i, i^{\prime}} e^{-\left(\lambda_{1} / 2\right)\left(\left|i-i^{\prime}\right|+\left|t-t^{\prime}\right|\right)} \\
& \leq 2 c_{1} K_{1} K_{N}(N T)^{-2}(N T)\left(\sum_{k=0}^{T} e^{-\left(\lambda_{1} / 2\right) k}\right)\left(\sum_{k=0}^{N} e^{-\left(\lambda_{1} / 2\right) k}\right) \\
& \leq 2 c_{1} K_{1} K_{N}(N T)^{-2}(N T)\left\{1-e^{-\left(\lambda_{1} / 2\right)}\right\}^{-2}=2 c_{1} K_{1} K_{N}\left\{1-e^{-\left(\lambda_{1} / 2\right)}\right\}^{-2}(N T)^{-1}=O\left\{K_{N}(N T)^{-1}\right\} .
\end{aligned}
$$

Thus, by Markov's inequality,

$$
\left\|(N T)^{-1} \sum_{t=1}^{T} \sum_{i=1}^{N} \psi_{i t}\right\|=O_{p}\left[\left\{K_{N}(N T)^{-1}\right\}^{1 / 2}\right] .
$$

Moreover, by the definition of $D_{N t, 1}$ given in (A.5), we have $D_{N t, 1}=N^{-1} \sum_{i=1}^{N} \psi_{i t}$. Therefore,

$$
\begin{aligned}
& \left|N^{-1} \sum_{i=1}^{N} T^{-1} \sum_{t=1}^{T} 1_{j+1}^{\top} \mathbb{B}\left(X_{i}\right) D_{N t, 1}\right|=\left|N^{-1} \sum_{i=1}^{N} 1_{j+1}^{\top} \mathbb{B}\left(X_{i}\right)(N T)^{-1} \sum_{t=1}^{T} \sum_{i=1}^{N} \psi_{i t}\right| \\
\leq & \|(N T)^{-1} \sum_{t=1}^{T} \sum_{i=1}^{N} \psi_{i t}||\left[\lambda_{\max }\left\{N^{-1} \sum_{i=1}^{N} B_{j}\left(X_{j i}\right) B_{j}\left(X_{j i}\right)^{\top}\right\}\right]^{1 / 2}=O_{p}\left[\left\{K_{N}(N T)^{-1}\right\}^{1 / 2}\right] .
\end{aligned}
$$

By (A.12) and $\log \left(K_{N} T\right) K_{N} N^{-1 / 2}=o(1)$, we have

$$
N^{-1} \sum_{i=1}^{N} T^{-1} \sum_{t=1}^{T}\left\{1_{j+1}^{\top} \mathbb{B}\left(X_{i}\right) D_{N t, 1}\right\}^{2}=\left\{O_{p}\left(\sqrt{\log K_{N} T} \sqrt{K_{N} / N}\right)^{2}\right\}=o_{p}\left(N^{-1 / 2}\right) .
$$

Therefore, the above results together with (A.15), (A.16) and (A.17) lead to

$$
\begin{aligned}
& \left|1 / \sqrt{N^{-1} \sum_{i=1}^{N}\left(T^{-1} \sum_{t=1}^{T} \widetilde{h}_{j t}\left(X_{j i}\right)\right)^{2}}-1 / \sqrt{N^{-1} \sum_{i=1}^{N}\left(T^{-1} \sum_{t=1}^{T} h_{j t}^{0}\left(X_{j i}\right)\right)^{2}}\right| \\
& =O_{p}\left[\left\{K_{N}(N T)^{-1}\right\}^{1 / 2}\right]+O_{p}\left(K_{N}^{-r}\right)+O_{p}\left(K_{N}^{3 / 2} N^{-3 / 4} \sqrt{\log N T}\right)+o_{p}\left(N^{-1 / 2}\right) .
\end{aligned}
$$


Denote $\varpi_{N T}=\sqrt{N^{-1} \sum_{i=1}^{N}\left(T^{-1} \sum_{t=1}^{T} \widetilde{h}_{j t}\left(X_{j i}\right)\right)^{2}}$ and $\varpi_{N T}^{0}=\sqrt{N^{-1} \sum_{i=1}^{N}\left(T^{-1} \sum_{t=1}^{T} h_{j t}^{0}\left(X_{j i}\right)\right)^{2}}$. Then

$$
\begin{aligned}
& T^{-1} \sum_{t=1}^{T}\left\{\widetilde{h}_{j t}\left(x_{j}\right) / \varpi_{N T}-h_{j t}^{0}\left(x_{j}\right) / \varpi_{N T}^{0}\right\} \\
& =T^{-1} \sum_{t=1}^{T}\left\{\widetilde{h}_{j t}\left(x_{j}\right) / \varpi_{N T}-h_{j t}^{0}\left(x_{j}\right) / \varpi_{N T}\right\}+T^{-1} \sum_{t=1}^{T}\left\{h_{j t}^{0}\left(x_{j}\right) / \varpi_{N T}-h_{j t}^{0}\left(x_{j}\right) / \varpi_{N T}^{0}\right\} \\
& =T^{-1} \sum_{t=1}^{T}\left\{\widetilde{h}_{j t}\left(x_{j}\right)-h_{j t}^{0}\left(x_{j}\right)\right\} / \varpi_{N T}+T^{-1} \sum_{t=1}^{T} h_{j t}^{0}\left(x_{j}\right)\left\{1 / \varpi_{N T}-1 / \varpi_{N T}^{0}\right\} .
\end{aligned}
$$

By the above result and Condition (C3), we have

$$
\begin{aligned}
& \sup _{x_{j} \in[a, b]}\left|T^{-1} \sum_{t=1}^{T} h_{j t}^{0}\left(x_{j}\right)\left\{1 / \varpi_{N T}-1 / \varpi_{N T}^{0}\right\}\right| \\
& =O_{p}\left[\left\{K_{N}(N T)^{-1}\right\}^{1 / 2}\right]+O_{p}\left(K_{N}^{-r}\right)+O_{p}\left(K_{N}^{3 / 2} N^{-3 / 4} \sqrt{\log N T}\right)+o_{p}\left(N^{-1 / 2}\right) .
\end{aligned}
$$

Moreover, (A.9) leads to that with probability approaching 1 , as $N \rightarrow \infty$,

$$
\begin{aligned}
& T^{-1} \sum_{t=1}^{T}\left\{\widetilde{h}_{j t}\left(x_{j}\right)-h_{j t}^{0}\left(x_{j}\right)\right\} / \varpi_{N T} \\
& =T^{-1} \sum_{t=1}^{T} 1_{j+1}^{\top} \mathbb{B}(x) D_{N t, 1} / \varpi_{N T}+T^{-1} \sum_{t=1}^{T} \eta_{N, j t}\left(x_{j}\right) / \varpi_{N T}=\Phi_{N T j, 1}\left(x_{j}\right)+\Phi_{N T j, 2}\left(x_{j}\right) .
\end{aligned}
$$

By (A.11) and (A.14),

$$
\begin{gathered}
\sup _{x_{j} \in[a, b]}\left|\Phi_{N T j, 2}\left(x_{j}\right)\right|=O_{p}\left(K_{N}^{-r}\right)+O_{p}\left(K_{N}^{2} N^{-3 / 4} \sqrt{\log N T}\right)+o_{p}\left(N^{-1 / 2}\right) \\
\left\{\int \Phi_{N T j, 2}\left(x_{j}\right)^{2} d x_{j}\right\}^{1 / 2}=O_{p}\left(K_{N}^{-r}\right)+O_{p}\left(K_{N}^{3 / 2} N^{-3 / 4} \sqrt{\log N T}\right)+o_{p}\left(N^{-1 / 2}\right) .
\end{gathered}
$$

By (A.14) and (A.18),

$$
\begin{aligned}
\sup _{x_{j} \in[a, b]}\left|\Phi_{N T j, 1}\left(x_{j}\right)\right| & \leq c_{h}^{-1}\left\{\left\|(N T)^{-1} \sum_{t=1}^{T} \sum_{i=1}^{N} \psi_{i t}\right\|^{2} \sup _{x_{j} \in[a, b]}\left\|B_{j}\left(x_{j}\right)\right\|^{2}\right\}^{1 / 2} \\
& =O_{p}\left\{K_{N}(N T)^{-1 / 2}\right\}, \\
\left\{\int \Phi_{N T j, 1}\left(x_{j}\right)^{2} d x_{j}\right\}^{1 / 2} & \leq c_{h}^{-1} C_{2}\left\{\left\|(N T)^{-1} \sum_{t=1}^{T} \sum_{i=1}^{N} \psi_{i t}\right\|^{2}\right\}^{1 / 2}=O_{p}\left\{K_{N}^{1 / 2}(N T)^{-1 / 2}\right\} .
\end{aligned}
$$

Hence, the results in Proposition 1 follow from the above results directly.

\subsection{Proofs of Theorems 1 and 2}

We first present the following several lemmas that will be used in the proofs of Theorems 1 and 2. We define the infeasible estimator $f_{t}^{*}=\left\{f_{u t}^{*},\left(f_{j t}^{*}, 1 \leq j \leq J\right)^{\top}\right\}^{\top}$ as the minimizer of

$$
\sum_{i=1}^{N} \rho_{\tau}\left(y_{i t}-f_{u t}-\sum_{j=1}^{J} g_{j}^{0}\left(X_{j i}\right) f_{j t}\right)
$$

Lemma 3. Under Conditions (C1), (C2), (C4), (C5) and (C6), we have as $N \rightarrow \infty$,

$$
\sqrt{N}\left(\Sigma_{N t}^{0}\right)^{-1 / 2}\left(f_{t}^{*}-f_{t}^{0}\right) \rightarrow \mathcal{N}\left(\mathbf{0}, \mathbf{I}_{J+1}\right)
$$

where $\Sigma_{N t}^{0}$ is given in (4.1). 
Proof. By Bahadur representation for the $\phi$-mixing case (see Babu (1989)), we have

$$
f_{t}^{*}-f_{t}^{0}=\Lambda_{N t}^{-1}\left\{N^{-1} \sum_{i=1}^{N} G_{i}^{0}\left(X_{i}\right)\left(\tau-I\left(\varepsilon_{i t}<0\right)\right)\right\}+v_{N t},
$$

and $\left\|v_{N t}\right\|=o_{p}\left(N^{-1 / 2}\right)$ for every $t$, where $\Lambda_{N t}=N^{-1} \sum_{i=1}^{N} p_{i}\left(0 \mid X_{i}, f_{t}\right) G_{i}^{0}\left(X_{i}\right) G_{i}^{0}\left(X_{i}\right)^{\top}$. By Conditions (C2) and (C5), we have that the eigenvalues of $\Lambda_{N t}^{0}$ are bounded away from zero and infinity. By similar reasoning to the proof for Theorem 2 in Lee and Robinson (2016), we have $\left\|\Lambda_{N t}^{-1}\right\|=O_{p}(1)$ and $\left\|\Lambda_{N t}-\Lambda_{N t}^{0}\right\|=o_{p}(1)$. Thus, the asymptotic distribution in Lemma 3 can be obtained directly by Condition (C6).

Recall that the initial estimator $\widehat{f}_{t}^{[0]}$ given in (3.2) is defined in the same way as $f_{t}^{*}$ with $g_{j}^{0}\left(X_{j i}\right)$ replaced by $\widehat{g}_{j}^{[0]}\left(X_{j i}\right)$ in (A.19). Then we have the following result for $\widehat{f}_{t}^{[0]}$.

Lemma 4. Let Conditions (C1)-(C5) hold. If, in addition, $K_{N}^{4} N^{-1}=o(1), K_{N}^{-r+2}(\log T)=$ $o(1)$ and $K_{N}^{-1}(\log N T)(\log N)^{4}=o(1)$, then for any $t$ there is a stochastically bounded sequence $\delta_{N, j t}$ such that as $N \rightarrow \infty$,

$$
\sqrt{N}\left(\widehat{f}_{t}^{[0]}-f_{t}^{*}-d_{N T} \delta_{N, t}\right)=o_{p}(1)
$$

where $\delta_{N, t}=\left(\delta_{N, j t}, 0 \leq j \leq J\right)^{\top}$ and $d_{N T}$ is given in (4.3).

Proof. Denote $g=\left\{g_{j}(\cdot), 1 \leq j \leq J\right\}$. Define

$$
\begin{aligned}
L_{N t}\left(f_{t}, g\right) & =N^{-1} \sum_{i=1}^{N} \rho_{\tau}\left(y_{i t}-f_{u t}-\sum_{j=1}^{J} g_{j}\left(X_{j i}\right) f_{j t}\right) \\
& -N^{-1} \sum_{i=1}^{N} \rho_{\tau}\left(y_{i t}-f_{u t}^{0}-\sum_{j=1}^{J} g_{j}\left(X_{j i}\right) f_{j t}^{0}\right),
\end{aligned}
$$

so that $f_{t}^{*}$ and $\widehat{f}_{t}^{[0]}$ are the minimizers of $L_{N t}\left(f_{t}, g^{0}\right)$ and $L_{N t}\left(f_{t}, \widehat{g}^{[0]}\right)$, respectively, where $\widehat{g}^{[0]}=\left\{\widehat{g}_{j}^{[0]}(\cdot), 1 \leq j \leq J\right\}$ and $g^{0}=\left\{g_{j}^{0}(\cdot), 1 \leq j \leq J\right\}$. According to the result on page 149 of de Boor (2001), for $g_{j}^{0}$ satisfying the smoothness condition given in (C2), there exists $\boldsymbol{\lambda}_{j}^{0} \in R^{K_{n}}$ such that $g_{j}^{0}\left(x_{j}\right)=\widetilde{g}_{j}^{0}\left(x_{j}\right)+r_{j}\left(x_{j}\right)$,

$$
\widetilde{g}_{j}^{0}\left(x_{j}\right)=B_{j}\left(x_{j}\right)^{\top} \boldsymbol{\lambda}_{j}^{0} \text { and } \sup _{j} \sup _{x_{j} \in[a, b]}\left|r_{j}\left(x_{j}\right)\right|=O\left(K_{N}^{-r}\right) .
$$

By Proposition 1, there exists $\boldsymbol{\lambda}_{j, N T} \in R^{K_{N}}$ such that $\widehat{g}_{j}^{[0]}\left(x_{j}\right)=B_{j}\left(x_{j}\right)^{\top} \boldsymbol{\lambda}_{j, N T}$ and $\| \boldsymbol{\lambda}_{j, N T}-$ $\boldsymbol{\lambda}_{j}^{0} \|=O_{p}\left(d_{N T}\right)+o_{p}\left(N^{-1 / 2}\right)$. Let $d_{N T}^{\prime}$ be a sequence satisfying $d_{N T}^{\prime}=o\left(N^{-1 / 2}\right)$ and let $d_{N T}^{*}=d_{N T}+d_{N T}^{\prime}$. In the following, we will show that

$$
\widetilde{f}_{t}-f_{t}^{0}-d_{N T} \delta_{N, t}=\Lambda_{N t}^{-1}\left\{N^{-1} \sum_{i=1}^{N} G_{i}^{0}\left(X_{i}\right)\left(\tau-I\left(\varepsilon_{i t}<0\right)\right)\right\}+o_{p}\left(N^{-1 / 2}\right),
$$

uniformly in $\left\|\boldsymbol{\lambda}_{j}-\boldsymbol{\lambda}_{j}^{0}\right\| \leq \widetilde{C} d_{N T}^{*}$ for some constant $0<\widetilde{C}<\infty$, where $\widetilde{f}_{t}$ is the minimizer of $L_{N t}\left(f_{t}, g\right)$ and $g_{j}\left(x_{j}\right)=B_{j}\left(x_{j}\right)^{\top} \boldsymbol{\lambda}_{j}$. Hence the result in Lemma 4 follows from (A.20) and (A.21). 
We have $\left\|\tilde{f}_{t}-f_{t}^{*}\right\|=o_{p}(1)$, since

$$
\begin{aligned}
& \left|L_{N t}\left(f_{t}, g\right)-L_{N t}\left(f_{t}, g^{0}\right)\right| \\
& \leq 2 N^{-1} \sum_{i=1}^{N}\left|\sum_{j=1}^{J}\left\{g_{j}\left(X_{j i}\right)-g_{j}^{0}\left(X_{j i}\right)\right\} f_{j t}\right|+2 N^{-1} \sum_{i=1}^{N}\left|\sum_{j=1}^{J}\left\{g_{j}\left(X_{j i}\right)-g_{j}^{0}\left(X_{j i}\right)\right\} f_{j t}^{0}\right| \\
& \leq C_{L} \widetilde{C}\left\{d_{N T}+o\left(N^{-1 / 2}\right)\right\}=o(1),
\end{aligned}
$$

for some constant $0<C_{L}<\infty$, where the first inequality follows from the fact that $\left|\rho_{\tau}(u-v)-\rho_{\tau}(u)\right| \leq 2|v|$. Thus $\left\|\tilde{f}_{t}-f_{t}^{0}\right\|=o_{p}(1)$. Let $\mathbf{X}=\left(X_{1}, \ldots, X_{N}\right)^{\top}, G_{i}\left(X_{i}\right)=$ $\left\{1, g_{1}\left(X_{1 i}\right), \ldots, g_{J}\left(X_{J i}\right)\right\}^{\top}$ and $\mathbf{F}=\left\{f_{1}, \ldots, f_{T}\right\}^{\top}$. Let

$$
\psi_{\tau}(\varepsilon)=\tau-I(\varepsilon<0)
$$

For $\boldsymbol{\lambda}_{j} \in R^{K_{n}}$ satisfying $\left\|\boldsymbol{\lambda}_{j}-\boldsymbol{\lambda}_{j}^{0}\right\| \leq \widetilde{C} d_{N T}^{*}$ and $f_{t}$ in a neighborhood of $f_{t}^{0}$, write

$$
\begin{aligned}
& L_{N t}\left(f_{t}, g\right)=E\left\{L_{N t}\left(f_{t}, g\right) \mid \mathbf{X}, \mathbf{F}\right\}-\left(f_{t}-f_{t}^{0}\right)^{\top}\left\{W_{N t, 1}-E\left(W_{N t, 1} \mid \mathbf{X}, \mathbf{F}\right)\right\} \\
& +W_{N t, 2}\left(f_{t}, g\right)-E\left(W_{N t, 2}\left(f_{t}, g\right) \mid \mathbf{X}, \mathbf{F}\right),
\end{aligned}
$$

where $g_{j}\left(x_{j}\right)=B_{j}\left(x_{j}\right)^{\top} \boldsymbol{\lambda}_{j}$, and

$$
\begin{aligned}
W_{N t, 1} & =N^{-1} \sum_{i=1}^{N} G_{i}\left(X_{i}\right) \psi_{\tau}\left(y_{i t}-f_{t}^{0 \top} G_{i}\left(X_{i}\right)\right), \\
W_{N t, 2}\left(f_{t}, g\right) & =N^{-1} \sum_{i=1}^{N}\left\{\rho_{\tau}\left(y_{i t}-f_{t}^{\top} G_{i}\left(X_{i}\right)\right)-\rho_{\tau}\left(y_{i t}-f_{t}^{0 \top} G_{i}\left(X_{i}\right)\right)\right. \\
& \left.+\left(f_{t}-f_{t}^{0}\right)^{\top} G_{i}\left(X_{i}\right) \psi_{\tau}\left(y_{i t}-f_{t}^{0 \top} G_{i}\left(X_{i}\right)\right)\right\} .
\end{aligned}
$$

In Lemma $\mathbf{S . 4}$ in the Supplemental Materials, we show that as $N \rightarrow \infty$,

$E\left\{L_{N t}\left(f_{t}, g\right) \mid \mathbf{X}, \mathbf{F}\right\}=-\left(f_{t}-f_{t}^{0}\right)^{\top} E\left(W_{N t, 1} \mid \mathbf{X}, \mathbf{F}\right)+\frac{1}{2}\left(f_{t}-f_{t}^{0}\right)^{\top} \Lambda_{N t}^{0}\left(f_{t}-f_{t}^{0}\right)+o_{p}\left(\left\|f_{t}-f_{t}^{0}\right\|^{2}\right)$, uniformly in $\left\|\boldsymbol{\lambda}_{j}-\boldsymbol{\lambda}_{j}^{0}\right\| \leq \widetilde{C} d_{N T}^{*}$ and $\left\|f_{t}-f_{t}^{0}\right\| \leq \varpi_{N}$, where $\varpi_{N}$ is any sequence of positive numbers satisfying $\varpi_{N}=o(1)$. Substituting this into (A.22), we have with probability approaching 1,

$$
\begin{aligned}
L_{N t}\left(f_{t}, g\right) & =-\left(f_{t}-f_{t}^{0}\right)^{\top} W_{N t, 1}+\frac{1}{2}\left(f_{t}-f_{t}^{0}\right)^{\top} \Lambda_{N}^{0}\left(f_{t}-f_{t}^{0}\right) \\
& +W_{N t, 2}\left(f_{t}, g\right)-E\left(W_{N t, 2}\left(f_{t}, g\right) \mid \mathbf{X}, \mathbf{F}\right)+o\left(\left\|f_{t}-f_{t}^{0}\right\|^{2}\right) .
\end{aligned}
$$

In Lemma $\underline{\text { S.5 }}$ in the Supplemental Materials, we show that

$$
W_{N t, 2}\left(f_{t}, g\right)-E\left(W_{N t, 2}\left(f_{t}, g\right) \mid \mathbf{X}, \mathbf{F}\right)=o_{p}\left(\left\|f_{t}-f_{t}^{0}\right\|^{2}+N^{-1}\right)
$$

uniformly in $\left\|\boldsymbol{\lambda}_{j}-\boldsymbol{\lambda}_{j}^{0}\right\| \leq \widetilde{C} d_{N T}$ and $\left\|f_{t}-f_{t}^{0}\right\| \leq \varpi_{N}$. Thus, we have $\widetilde{f}_{t}-f_{t}^{0}=\left(\Lambda_{N t}^{0}\right)^{-1} W_{N t, 1}+$ $o_{p}\left(N^{-1 / 2}\right)$. Since $\left\|\left(\Lambda_{N t}^{0}\right)^{-1}-\left(\Lambda_{N t}\right)^{-1}\right\|=o_{p}(1)$, we have

$$
\widetilde{f}_{t}-f_{t}^{0}=\Lambda_{N t}^{-1} W_{N t, 1}+o_{p}\left(N^{-1 / 2}\right) .
$$


In Lemma S.6 in the Supplemental Materials, we show that for any $t$ there is a stochastically bounded sequence $\delta_{N, j t}$ such that as $N \rightarrow \infty$,

$$
W_{N t, 1}=N^{-1} \sum_{i=1}^{N} G_{i}^{0}\left(X_{i}\right) \psi_{\tau}\left(\varepsilon_{i t}\right)+d_{N T} \delta_{N, t}+o_{p}\left(N^{-1 / 2}\right)
$$

where $\delta_{N, t}=\left(\delta_{N, j t}, 0 \leq j \leq J\right)^{\top}$ and $g_{j}\left(x_{j}\right)=B_{j}\left(x_{j}\right)^{\top} \boldsymbol{\lambda}_{j}$, uniformly in $\left\|\boldsymbol{\lambda}_{j}-\boldsymbol{\lambda}_{j}^{0}\right\| \leq \widetilde{C} d_{N T}^{*}$. Hence, result (A.21) follows from (A.25) and (A.26) directly. Then the proof is complete.

Let $\boldsymbol{\lambda}=\left(\boldsymbol{\lambda}_{1}^{\top}, \ldots, \boldsymbol{\lambda}_{J}^{\top}\right)^{\top}$. For given $\widehat{f}^{[0]}$, we obtain

$\widehat{\boldsymbol{\lambda}}^{[1]}=\left(\boldsymbol{\lambda}_{1}^{[1] \boldsymbol{\top}}, \ldots, \boldsymbol{\lambda}_{J}^{[1] \boldsymbol{\top}}\right)^{\boldsymbol{\top}}=\arg \min _{\boldsymbol{\lambda}}\left\{(N T)^{-1} \sum_{i=1}^{N} \sum_{t=1}^{T} \rho_{\tau}\left(y_{i t}-\widehat{f}_{u t}^{[0]}-\sum_{j=1}^{J} B_{j}\left(X_{j i}\right)^{\top} \boldsymbol{\lambda}_{j} \widehat{f}_{j t}^{[0]}\right)\right\}$.

Let $\widehat{g}_{j}^{*[1]}\left(x_{j}\right)=B_{j}\left(x_{j}\right)^{\top} \widehat{\boldsymbol{\lambda}}_{j}^{[1]}$. The estimator for $g_{j}\left(x_{j}\right)$ at the $1^{\text {st }}$ step is

$$
\widehat{g}_{j}^{[1]}\left(x_{j}\right)=\widehat{g}_{j}^{*[1]}\left(x_{j}\right) / \sqrt{N^{-1} \sum_{i=1}^{N} \widehat{g}_{j}^{*[1]}\left(X_{j i}\right)^{2}} .
$$

We define the infeasible estimator of $\boldsymbol{\lambda}$ as

$$
\boldsymbol{\lambda}^{*}=\left(\boldsymbol{\lambda}_{1}^{* \top}, \ldots, \boldsymbol{\lambda}_{J}^{* \top}\right)^{\top}=\arg \min _{\boldsymbol{\lambda}}\left\{(N T)^{-1} \sum_{i=1}^{N} \sum_{t=1}^{T} \rho_{\tau}\left(y_{i t}-f_{u t}^{0}-\sum_{j=1}^{J} B_{j}\left(X_{j i}\right)^{\top} \boldsymbol{\lambda}_{j} f_{j t}^{0}\right)\right\} .
$$

Let $g_{j}^{*}\left(x_{j}\right)=B_{j}\left(x_{j}\right)^{\top} \boldsymbol{\lambda}_{j}^{*}$ and $\widetilde{g}_{j}^{*}\left(x_{j}\right)=g_{j}^{*}\left(x_{j}\right) / \sqrt{N^{-1} \sum_{i=1}^{N} g_{j}^{*}\left(X_{j i}\right)^{2}}$.

Lemma 5. Let Conditions (C1)-(C5) hold. If, in addition, $K_{N}^{4} N^{-1}=o(1), K_{N}^{-r+2}(\log T)=$ $o(1)$ and $K_{N}^{-1}(\log N T)(\log N)^{4}=o(1)$, then for every $1 \leq j \leq J$,

$$
\left[\int\left\{\widetilde{g}_{j}^{*}\left(x_{j}\right)-g_{j}^{0}\left(x_{j}\right)\right\}^{2} d x_{j}\right]^{1 / 2}=O_{p}\left(K_{N}^{1 / 2}(N T)^{-1 / 2}+K_{N}^{-r}\right),
$$

and

$$
\int\left\{\widehat{g}_{j}^{[1]}\left(x_{j}\right)\left(x_{j}\right)-\widetilde{g}_{j}^{*}\left(x_{j}\right)\right\}^{2} d x_{j}=O_{p}\left(d_{N T}^{2}\right)+o_{p}\left(N^{-1 / 2}\right) .
$$

Therefore, for every $1 \leq j \leq J$,

$$
\int\left\{\widehat{g}_{j}^{[1]}\left(x_{j}\right)-g_{j}^{0}\left(x_{j}\right)\right\}^{2} d x_{j}=O_{p}\left(d_{N T}^{2}\right)+o_{p}\left(N^{-1 / 2}\right) .
$$

Proof. Denote $\widetilde{g}^{0}(x)=\left\{\widetilde{g}_{j}^{0}\left(x_{j}\right), 1 \leq j \leq J\right\}^{\top}$ and $g^{*}(x)=\left\{g_{j}^{*}\left(x_{j}\right), 1 \leq j \leq J\right\}^{\top}$. Let $\boldsymbol{\lambda}^{0}=$ $\left(\boldsymbol{\lambda}_{1}^{0 \top}, \ldots, \boldsymbol{\lambda}_{J}^{0 \top}\right)^{\top}$. Let $\mathbb{B}^{*}(x)=\left[\operatorname{diag}\left[B_{1}\left(x_{1}\right)^{\top}, \ldots, B_{J}\left(x_{J}\right)^{\top}\right]\right]_{J \times J K_{N}}$. Then $\mathbb{B}^{*}(x) \boldsymbol{\lambda}^{*}=g^{*}(x)$ and $\mathbb{B}^{*}(x) \boldsymbol{\lambda}^{0}=\widetilde{g}^{0}(x)$. Let $Q_{i t}^{0}=\left\{B_{j}\left(X_{j i}\right)^{\top} f_{j t}^{0}, 1 \leq j \leq J\right\}^{\top}$,

$$
\Psi_{N T}=(N T)^{-1} \sum_{i=1}^{N} \sum_{t=1}^{T} f_{\varepsilon}\left(0 \mid X_{i}, f_{t}\right) Q_{i t}^{0} Q_{i t}^{0 \top},
$$

and $r_{j, i t}^{*}=r_{j}\left(X_{j i}\right) f_{j t}^{0}$. Moreover, define

$$
\begin{aligned}
U_{N T, 1} & =(N T)^{-1} \sum_{i=1}^{N} \sum_{t=1}^{T} Q_{i t}^{0}\left(\tau-I\left(\varepsilon_{i t}<0\right)\right), \\
U_{N T, 2} & =(N T)^{-1} \sum_{i=1}^{N} \sum_{t=1}^{T} Q_{i t}^{0} f_{\varepsilon}\left(0 \mid X_{i}, f_{t}\right)\left(\sum_{j=1}^{J} r_{j, i t}^{*}\right) .
\end{aligned}
$$


By the same procedure as the proof of Lemma 2, for $K_{N}^{4}(\log (N T))^{2}(N T)^{-1}=o(1)$, we obtain the Bahadur representation for $\boldsymbol{\lambda}^{*}-\boldsymbol{\lambda}^{0}$ as

$$
\boldsymbol{\lambda}^{*}-\boldsymbol{\lambda}^{0}=\Psi_{N T}^{-1}\left(U_{N, 1}+U_{N, 2}\right)+R_{N T}^{*},
$$

and the remaining term $R_{N T}^{*}$ satisfies

$$
\begin{aligned}
\left\|R_{N T}^{*}\right\| & =O_{p}\left(K_{N}^{3 / 2}(N T)^{-1}+K_{N}^{3 / 2}(N T)^{-3 / 4} \sqrt{\log (N T)}+K_{N}^{1 / 2-2 r}+(N T)^{-1 / 2} K_{N}^{-r / 2+1 / 2}\right) \\
& =O_{p}\left(K_{N}^{3 / 2}(N T)^{-3 / 4} \sqrt{\log (N T)}+K_{N}^{1 / 2-2 r}\right)+o_{p}\left((N T)^{-1 / 2}\right) .
\end{aligned}
$$

By (A.32) and following the same reasoning as the proof for (A.13), we have $\sup _{x_{j} \in[a, b]} \mid g_{j}^{*}\left(x_{j}\right)-$ $g_{j}^{0}\left(x_{j}\right) \mid=O_{p}\left(K_{N}(N T)^{-1 / 2}+K_{N}^{-r}\right),\left[\int\left\{g_{j}^{*}\left(x_{j}\right)-g_{j}^{0}\left(x_{j}\right)\right\}^{2} d x_{j}\right]^{1 / 2}=O_{p}\left(K_{N}^{1 / 2}(N T)^{-1 / 2}+K_{N}^{-r}\right)$, and $\left[N^{-1} \sum_{i=1}^{N}\left\{g_{j}^{*}\left(X_{j i}\right)-g_{j}^{0}\left(X_{j i}\right)\right\}^{2}\right]^{1 / 2}=O_{p}\left(K_{N}^{1 / 2}(N T)^{-1 / 2}+K_{N}^{-r}\right)$. Therefore, we have

$$
\left\{\sqrt{N^{-1} \sum_{i=1}^{N} g_{j}^{*}\left(X_{j i}\right)^{2}}\right\}^{-1}-\left\{\sqrt{N^{-1} \sum_{i=1}^{N} g_{j}^{0}\left(X_{j i}\right)^{2}}\right\}^{-1}=O_{p}\left(K_{N}^{1 / 2}(N T)^{-1 / 2}+K_{N}^{-r}\right),
$$

and thus

$$
\begin{aligned}
\sup _{x_{j} \in[a, b]}\left|\widetilde{g}_{j}^{*}\left(x_{j}\right)-g_{j}^{0}\left(x_{j}\right)\right| & =O_{p}\left(K_{N}(N T)^{-1 / 2}+K_{N}^{-r}\right), \\
{\left[\int\left\{\widetilde{g}_{j}^{*}\left(x_{j}\right)-g_{j}^{0}\left(x_{j}\right)\right\}^{2} d x_{j}\right]^{1 / 2} } & =O_{p}\left(K_{N}^{1 / 2}(N T)^{-1 / 2}+K_{N}^{-r}\right) .
\end{aligned}
$$

Then the result (A.27) is proved. Define

$$
\begin{aligned}
L_{N T}^{*}(f, \boldsymbol{\lambda}) & =(N T)^{-1} \sum_{i=1}^{N} \sum_{t=1}^{T} \rho_{\tau}\left(y_{i t}-f_{u t}-\sum_{j=1}^{J} B_{j}\left(X_{j i}\right)^{\top} \boldsymbol{\lambda}_{j} f_{j t}\right) \\
& -(N T)^{-1} \sum_{i=1}^{N} \sum_{t=1}^{T} \rho_{\tau}\left(y_{i t}-f_{u t}-\sum_{j=1}^{J} B_{j}\left(X_{j i}\right)^{\top} \boldsymbol{\lambda}_{j}^{0} f_{j t}\right) .
\end{aligned}
$$

Hence, $\widehat{\boldsymbol{\lambda}}^{[1]}$ and $\boldsymbol{\lambda}^{*}$ are the minimizers of $L_{N T}^{*}\left(\widehat{f}^{[0]}, \boldsymbol{\lambda}\right)$ and $L_{N T}^{*}\left(f^{0}, \boldsymbol{\lambda}\right)$, respectively. In Lemma S.7 in the Supplemental Materials, we show that

$$
\left\|\widehat{\lambda}^{[1]}-\lambda^{0}-\Psi_{N T}^{-1} U_{N, 1}\right\|=O_{p}\left(d_{N T}\right)+o_{p}\left(N^{-1 / 2}\right) .
$$

Hence, by (A.32), (A.33) and $\left\|\Psi_{N T}^{-1} U_{N, 2}\right\|=O\left(K_{N}^{-r}\right)$, we have

$$
\left\|\widehat{\boldsymbol{\lambda}}^{[1]}-\boldsymbol{\lambda}^{*}\right\|=O_{p}\left(d_{N T}\right)+o_{p}\left(N^{-1 / 2}\right) .
$$

Then we have $\int\left\{\widehat{g}_{j}^{*[1]}\left(x_{j}\right)-g_{j}^{*}\left(x_{j}\right)\right\}^{2} d x_{j}=O_{p}\left(d_{N T}^{2}\right)+o_{p}\left(N^{-1}\right)$ and $N^{-1} \sum_{i=1}^{N}\left\{\widehat{g}_{j}^{*[1]}\left(X_{j i}\right)-\right.$ $\left.g_{j}^{*}\left(X_{j i}\right)\right\}^{2}=O_{p}\left(d_{N T}^{2}\right)+o_{p}\left(N^{-1}\right)$. Thus,

$$
\left\{\sqrt{N^{-1} \sum_{i=1}^{N} \widehat{g}_{j}^{*[1]}\left(X_{j i}\right)^{2}}\right\}^{-1}-\left\{\sqrt{N^{-1} \sum_{i=1}^{N} g_{j}^{*}\left(X_{j i}\right)^{2}}\right\}^{-1}=O_{p}\left(d_{N T}\right)+o_{p}\left(N^{-1 / 2}\right) .
$$

Therefore, the result (A.28) follows from the above results directly. 
Proofs of Theorems 1 and 2. Based on (A.29) in Lemma 5, the result in Lemma 4 holds for $\widehat{f}_{t}^{[1]}$ with a different bounded sequence. Then the result (A.29) in Lemma 5 holds for $\widehat{g}_{j}^{[2]}\left(x_{j}\right)$. This process can be continued for any finite number of iterations. By assuming that the algorithm in Section 3.1 converges at the $(i+1)^{\text {th }}$ step for any finite number $i$, the results in Lemmas 4 and 5 hold for $\widehat{f}_{t}=\widehat{f}_{t}^{[i+1]}$ and $\widehat{g}_{j}=\widehat{g}_{j}^{[i+1]}\left(x_{j}\right)$. Hence, Theorem 1 for $\widehat{f_{t}}$ follows from Lemmas 3 and 4 , directly, and Theorem 2 for $\widehat{g}_{j}$ is proved by using Lemma 5 .

\subsection{Proofs of Theorem 3}

Proof. We prove the consistency of $\widehat{\Lambda}_{N t}$. Define

$$
\widetilde{\Lambda}_{N t}=(N h)^{-1} \sum_{i=1}^{N} K\left(\frac{y_{i t}-\left(f_{u t}^{0}+\sum_{j=1}^{J} g_{j}^{0}\left(X_{j i}\right) f_{j t}^{0}\right)}{h}\right) G_{i}^{0}\left(X_{i}\right) G_{i}^{0}\left(X_{i}\right)^{\top},
$$

and

$$
\widehat{\Lambda}_{N t}=(N h)^{-1} \sum_{i=1}^{N} K\left(\frac{y_{i t}-\left(\widehat{f}_{u t}+\sum_{j=1}^{J} \widehat{g}_{j}\left(X_{j i}\right) \widehat{f}_{j t}\right)}{h}\right) \widehat{G}_{i}\left(X_{i}\right) \widehat{G}_{i}\left(X_{i}\right)^{\top} .
$$

We will show $\left\|\widehat{\Lambda}_{N t}-\widetilde{\Lambda}_{N t}\right\|=o_{p}(1)$ and $\left\|\widetilde{\Lambda}_{N t}-\Lambda_{N t}^{0}\right\|=o_{p}(1)$, respectively. Let $\widehat{d}_{i t}\left(X_{i}\right)=$ $\left\{\widehat{f}_{u t}+\sum_{j=1}^{J} \widehat{g}_{j}\left(X_{j i}\right) \widehat{f}_{j t}\right\}-\left\{f_{u t}^{0}+\sum_{j=1}^{J} g_{j}^{0}\left(X_{j i}\right) f_{j t}^{0}\right\}$. Then,

$$
\widehat{\Lambda}_{N t}-\widetilde{\Lambda}_{N t}=D_{N t, 1}+D_{N t, 2}
$$

where

$$
\begin{aligned}
& D_{N t, 1}=(2 N h)^{-1} \sum_{i=1}^{N}\left\{I\left(\left|\varepsilon_{i t}\right| \leq h\right)-I\left(\left|\varepsilon_{i t}-\widehat{d}_{i t}\left(X_{i}\right)\right| \leq h\right)\right\} G_{i}^{0}\left(X_{i}\right) G_{i}^{0}\left(X_{i}\right)^{\top}, \\
& D_{N t, 2}=(2 N h)^{-1} \sum_{i=1}^{N} I\left(\left|\varepsilon_{i t}-\widehat{d}_{i t}\left(X_{i}\right)\right| \leq h\right)\left\{\widehat{G}_{i}\left(X_{i}\right) \widehat{G}_{i}\left(X_{i}\right)^{\top}-G_{i}^{0}\left(X_{i}\right) G_{i}^{0}\left(X_{i}\right)^{\top}\right\} .
\end{aligned}
$$

Since there exist some constants $0<c_{f}, c_{1}<\infty$ such that with probability approaching 1 ,

$$
E\left\{\widehat{d}_{i t}\left(X_{i}\right)\right\}^{2}=\int \widehat{d}_{i t}^{2}(x) f_{X_{i}}(x) d x \leq c_{f} \int \widehat{d}_{i t}^{2}(x) d x \leq c_{1} \phi_{N T}^{2}+o\left(N^{-1}\right)
$$

where $\phi_{N T}$ is given in (4.2), and the last inequality follows from the result in Theorem 2 , then there exists some constant $0<c<\infty$ such that with probability approaching 1 ,

$$
\begin{aligned}
E\left\|\widehat{\Lambda}_{N t}-\widetilde{\Lambda}_{N t}\right\| & \leq c(2 N h)^{-1} \sum_{i=1}^{N} E\left|\widehat{d}_{i t}\left(X_{i}\right)\right| \times\left\|G_{i}^{0}\left(X_{i}\right) G_{i}^{0}\left(X_{i}\right)^{\top}\right\| \\
& \left.\leq c(2 N h)^{-1} \sum_{i=1}^{N} E\left\{\widehat{d}_{i t}\left(X_{i}\right)\right\}^{2} E\left\|G_{i}^{0}\left(X_{i}\right) G_{i}^{0}\left(X_{i}\right)^{\top}\right\|^{2}\right\}^{1 / 2} \\
& \leq c c_{1}^{1 / 2}(2 N h)^{-1}\left(\sqrt{K_{N} /(N T)}+K_{N}^{3 / 2} N^{-3 / 4} \sqrt{\log N}+K_{N}^{-r}\right) \times \\
& \sum_{i=1}^{N}\left\{E\left\|G_{i}^{0}\left(X_{i}\right) G_{i}^{0}\left(X_{i}\right)^{\top}\right\|^{2}\right\}^{1 / 2} .
\end{aligned}
$$


By Condition (C3), we have $\sup _{x_{j} \in[a, b]}\left|g_{j}^{0}\left(x_{j}\right)\right| \leq C^{\prime}$ for all $j$, for any vector $\mathbf{a} \in R^{J+1}$ and $\|\mathbf{a}\|^{2}=1$, we have

$$
\begin{aligned}
\mathbf{a}^{\top} G_{i}^{0}\left(X_{i}\right) G_{i}^{0}\left(X_{i}\right)^{\top} \mathbf{a} & =\left\{a_{0}+\sum_{j=1}^{J} g_{j}^{0}\left(X_{j i}\right) a_{j}\right\}^{2} \leq(J+1)\left\{a_{0}^{2}+g_{j}^{0}\left(X_{j i}\right)^{2} a_{j}^{2}\right\} \\
& \leq(J+1)\left\{a_{0}^{2}+\left(C^{\prime}\right)^{2} a_{j}^{2}\right\} \leq C_{a}
\end{aligned}
$$

for some constant $0<C_{a}<\infty$. Hence, $\left\|G_{i}^{0}\left(X_{i}\right) G_{i}^{0}\left(X_{i}\right)^{\top}\right\| \leq C_{a}$, and thus we have

$$
\begin{aligned}
& E\left\|\widehat{\Lambda}_{N t}-\widetilde{\Lambda}_{N t}\right\| \leq c c_{1}^{1 / 2}(2 N h)^{-1}\left(\phi_{N T}+o\left(N^{-1 / 2}\right)\right) \sum_{i=1}^{N} C_{a} \\
& =2^{-1} c c_{1}^{1 / 2} C_{a} h^{-1}\left(\phi_{N T}+o\left(N^{-1 / 2}\right)\right)=o(1)
\end{aligned}
$$

by the assumption that $h^{-1} \phi_{N T}=o(1)$ and $h^{-1} N^{-1 / 2}=O(1)$. Hence, we have $\left\|D_{N t, 1}\right\|=$ $o_{p}(1)$. Moreover, for any vector $\mathbf{a} \in R^{J+1}$ and $\|\mathbf{a}\|^{2}=1$, we have with probability approaching 1 , there exists a constant $0<C<\infty$ such that

$$
\begin{aligned}
\left|\mathbf{a}^{\top} D_{N t, 2} \mathbf{a}\right| & \leq(2 N h)^{-1} \sum_{i=1}^{N}\left|\left\{a_{0}+\sum_{j=1}^{J} \widehat{g}_{j}\left(X_{j i}\right) a_{j}\right\}^{2}-\left\{a_{0}+\sum_{j=1}^{J} g_{j}^{0}\left(X_{j i}\right) a_{j}\right\}^{2}\right| \\
& \leq C(2 N h)^{-1} \sum_{i=1}^{N} \sum_{j=1}^{J}\left|\left\{\widehat{g}_{j}\left(X_{j i}\right)-g_{j}^{0}\left(X_{j i}\right)\right\} a_{j}\right| \\
& \leq C(2 h)^{-1} \sum_{j=1}^{J}\left\{N^{-1} \sum_{i=1}^{N}\left\{\widehat{g}_{j}\left(X_{j i}\right)-g_{j}^{0}\left(X_{j i}\right)\right\}^{2} a_{j}^{2}\right\}^{1 / 2} \\
& =O\left(h^{-1}\right)\left\{O\left(\phi_{N T}\right)+o\left(N^{-1 / 2}\right)\right\}=o(1) .
\end{aligned}
$$

Hence, we have $\left\|D_{N t, 2}\right\|=o_{p}(1)$. Therefore, $\left\|\widehat{\Lambda}_{N t}-\widetilde{\Lambda}_{N t}\right\| \leq\left\|D_{N t, 1}\right\|+\left\|D_{N t, 2}\right\|=o_{p}(1)$. Next, we will show $\left\|\widetilde{\Lambda}_{N t}-\Lambda_{N t}^{0}\right\|=o_{p}(1)$. Since

$$
\begin{aligned}
& \left|E\left\{(2 h)^{-1} I\left(\left|\varepsilon_{i t}\right| \leq h\right)-p_{i}\left(0 \mid X_{i}\right) \mid X_{i}, f_{t}\right\}\right| \\
& =\left|(2 h)^{-1} h\left\{p_{i}\left(h^{*} \mid X_{i}, f_{t}\right)+p_{i}\left(-h^{* *} \mid X_{i}, f_{t}\right)\right\}-p_{i}\left(0 \mid X_{i}, f_{t}\right)\right| \\
& =\left|2^{-1}\left[\left\{p_{i}\left(h^{*} \mid X_{i}, f_{t}\right)-p_{i}\left(0 \mid X_{i}, f_{t}\right)\right\}+\left\{p_{i}\left(-h^{* *} \mid X_{i}, f_{t}\right)-p_{i}\left(0 \mid X_{i}, f_{t}\right)\right\}\right]\right| \leq c^{\prime} h
\end{aligned}
$$

for some constant $0<c^{\prime}<\infty$, where $h^{*}$ and $h^{* *}$ are some values between 0 and $h$, and the last inequality follows from Condition (C2), then by the above result and Condition (C5),

$$
\begin{aligned}
\left\|E\left(\widetilde{\Lambda}_{N t}-\Lambda_{N t}^{0}\right)\right\| & =\left\|N^{-1} \sum_{i=1}^{N} E\left[\left\{(2 h)^{-1} I\left(\left|\varepsilon_{i t}\right| \leq h\right)-p_{i}\left(0 \mid X_{i}, f_{t}\right)\right\} G_{i}^{0}\left(X_{i}\right) G_{i}^{0}\left(X_{i}\right)^{\top}\right]\right\| \\
& \leq c^{\prime} h\left\|N^{-1} \sum_{i=1}^{N} E Q_{i}^{0}\left(X_{i}\right) G_{i}^{0}\left(X_{i}\right)^{\top}\right\|=O(h)=o(1) .
\end{aligned}
$$

Moreover, by Conditions (C1), we have $E\left\{I\left(\left|\varepsilon_{i t}\right| \leq h\right)\right\} \leq 2 C^{*} h$ for some constant $C^{*} \in$ $(0, \infty)$, and then for any vector $\mathbf{a} \in R^{(J+1)}$ with $\|\mathbf{a}\|=1$, by Conditions (C1), (C2) and (C3), 
we have

$$
\begin{aligned}
& \operatorname{var}\left(\mathbf{a}^{\top} \widetilde{\Lambda}_{N t} \mathbf{a}\right) \\
& =(2 N h)^{-2} \operatorname{var}\left(\sum_{i=1}^{N} I\left(\left|\varepsilon_{i t}\right| \leq h\right)\left\{a_{0}+\sum_{j=1}^{J} g_{j}^{0}\left(X_{j i}\right) a_{j}\right\}^{2}\right) \\
& \leq(2 N h)^{-2} \sum_{i, i^{\prime}} 2\left\{\phi\left(\left|i-i^{\prime}\right|\right)\right\}^{1 / 2} \times \\
& \left(E\left[I\left(\left|\varepsilon_{i t}\right| \leq h\right)\left\{a_{0}+\sum_{j=1}^{J} g_{j}^{0}\left(X_{j i}\right) a_{j}\right\}^{4}\right]\right)^{1 / 2}\left(E\left[I\left(\left|\varepsilon_{i^{\prime} t^{\prime}}\right| \leq h\right)\left\{a_{0}+\sum_{j=1}^{J} g_{j}^{0}\left(X_{j i^{\prime}}\right) a_{j}\right\}^{4}\right]\right)^{1 / 2} \\
& \leq(J+1)^{2}\left\{a_{0}^{2}+C^{\prime 2} a_{j}^{2}\right\}(2 N h)^{-2}\left(2 C^{*} h\right)^{2} \sum_{i, i^{\prime}} 2\left\{\phi\left(\left|i-i^{\prime}\right|\right)\right\}^{1 / 2} \\
& \leq(J+1)^{2}\left\{a_{0}^{2}+C^{\prime 2} a_{j}^{2}\right\} N^{-2} 2 C^{* 2} K_{1} \sum_{i, i^{\prime}} e^{-\left(\lambda_{1} / 2\right)\left(\left|i-i^{\prime}\right|\right)} \\
& \leq(J+1)^{2}\left\{a_{0}^{2}+C^{\prime 2} a_{j}^{2}\right\} 2 C^{* 2} K_{1} N^{-1}\left\{1-e^{-\left(\lambda_{1} / 2\right)}\right\}=O\left(N^{-1}\right)=o(1) .
\end{aligned}
$$

By (A.35) and (A.36), we have $\left\|\widetilde{\Lambda}_{N t}-\Lambda_{N t}^{0}\right\|=o_{p}(1)$. Hence, $\left\|\widehat{\Lambda}_{N t}-\Lambda_{N t}^{0}\right\| \leq\left\|\widehat{\Lambda}_{N t}-\widetilde{\Lambda}_{N t}\right\|+$ $\left\|\widetilde{\Lambda}_{N t}-\Lambda_{N t}^{0}\right\|=o_{p}(1)$.

\subsection{Proofs of Theorem 4}

Proof. Let $S_{[r N] t}=\sum_{i=1}^{[r N]} G_{i}^{0}\left(X_{i}\right)\left(\tau-I\left(\varepsilon_{i t}<0\right)\right)$, where $[a]$ denotes the largest integer no greater than $a$. Let $M=b N$. Define $\Lambda_{N t}(r)=N^{-1} \sum_{i=1}^{[r N]} p_{i}\left(0 \mid X_{i}, f_{t}\right) G_{i}^{0}\left(X_{i}\right) G_{i}^{0}\left(X_{i}\right)^{\top}$, $\digamma_{N t}(r)=N^{-1 / 2} S_{[r N] t}$, and

$$
D_{b N}(r)=N^{2}\left(K^{*}\left(\frac{[r N]+1}{b N}\right)-K^{*}\left(\frac{[r N]}{b N}\right)\right)-\left(K^{*}\left(\frac{[r N]}{b N}\right)-K^{*}\left(\frac{[r N]-1}{b N}\right)\right) .
$$

Denote $K_{i j}^{*}=K^{*}\left(\frac{i-j}{b N}\right)$, and $\widehat{w}_{N t}=\frac{\tau(1-\tau)}{N} \sum_{i=1}^{N} \widehat{G}_{i}\left(X_{i}\right) \widehat{G}_{i}\left(X_{i}\right)^{\top}-N^{-1} \sum_{i=1}^{N} \widehat{v}_{i t} \widehat{v}_{i t}^{\top}$. Then

$$
\begin{aligned}
\widehat{\Omega}_{N t, N} & =N^{-1} \sum_{i=1}^{N} \sum_{j=1}^{N} \widehat{v}_{i t} K_{i j}^{*} \widehat{v}_{j t}^{\top}+\widehat{w}_{N t} \\
& =N^{-1} \sum_{i=1}^{N}\left(\widehat{v}_{i t} \sum_{j=1}^{N} K_{i j}^{*} \widehat{v}_{j t}^{\top}\right)+\widehat{w}_{N t} .
\end{aligned}
$$

Define $\widehat{S}_{n t}=\sum_{i=1}^{n} \widehat{v}_{i t}$. By the assumptions in Theorem 1, $\phi_{N T} N^{1 / 2}=o(1)$ and by the results in Lemmas 3.5, we have

$$
\begin{gathered}
\widehat{f_{t}}-f_{t}^{0}=\Lambda_{N t}^{-1}\left\{N^{-1} \sum_{i=1}^{N} G_{i}^{0}\left(X_{i}\right)\left(\tau-I\left(\varepsilon_{i t}<0\right)\right)\right\}+o_{p}\left(N^{-1 / 2}\right), \\
\sup _{x_{j} \in \mathcal{X}_{j}}\left|\widehat{g}_{j}\left(x_{j}\right)-g_{j}^{0}\left(x_{j}\right)\right|=O_{p}\left(\phi_{N T}\right)+o_{p}\left(N^{-1 / 2}\right)=o_{p}\left(N^{-1 / 2}\right) .
\end{gathered}
$$

Let $r \in(0,1]$. Let $\widetilde{S}_{[r N] t}=\sum_{i=1}^{[r N]} G_{i}^{0}\left(X_{i}\right)\left(\tau-I\left(\widehat{\varepsilon}_{i t}^{0}<0\right)\right)$, where $\widehat{\varepsilon}_{i t}^{0}=y_{i t}-\left\{\widehat{f}_{u t}+\right.$ $\left.\sum_{j=1}^{J} g_{j}^{0}\left(X_{j i}\right) \widehat{f}_{j t}\right\}$. By Lemma $\underline{\text { S.6.6, we have }}$

$$
\left\|N^{-1 / 2} \widehat{S}_{[r N] t}-N^{-1 / 2} \widetilde{S}_{[r N] t}\right\|=o_{p}(1)
$$


For any given $f_{t} \in R^{J+1}$, define $S_{[r N] t}\left(f_{t}\right)=\sum_{i=1}^{[r N]} G_{i}^{0}\left(X_{i}\right)\left(\tau-I\left(\varepsilon_{i t}\left(f_{t}\right)<0\right)\right)$, where $\varepsilon_{i t}\left(f_{t}\right)=y_{i t}-\left\{f_{u t}+\sum_{j=1}^{J} g_{j}^{0}\left(X_{j i}\right) f_{j t}\right\}$. Following similar arguments to the proof in Lemma S.8, we have

$\sup _{\left\|f_{t}-f_{t}^{0}\right\| \leq C\left(d_{N T}+N^{-1 / 2}\right)}\left\|N^{-1 / 2}\left[S_{[r N] t}\left(f_{t}\right)-S_{[r N] t}\left(f_{t}^{0}\right)-E\left[\left\{S_{[r N] t}\left(f_{t}\right)-S_{[r N] t}\left(f_{t}^{0}\right)\right\} \mid \mathbf{X}, \mathbf{F}\right]\right]\right\|=o_{p}(1)$.

Moreover,

$$
\begin{aligned}
& N^{-1 / 2} E\left[\left\{S_{[r N] t}\left(f_{t}\right)-S_{[r N] t}\left(f_{t}^{0}\right)\right\} \mid \mathbf{X}, \mathbf{F}\right] \\
& =\sum_{i=1}^{[r N]} G_{i}^{0}\left(X_{i}\right) E\left[\left(I\left(\varepsilon_{i t}\left(f_{t}^{0}\right)<0\right)-I\left(\varepsilon_{i t}\left(f_{t}\right)<0\right)\right) \mid X_{i}, f_{t}\right],
\end{aligned}
$$

and thus by Taylor's expansion, we have

$$
\begin{gathered}
\| N^{-1 / 2} E\left[\left\{S_{[r N] t}\left(f_{t}\right)-S_{[r N] t}\left(f_{t}^{0}\right)\right\} \mid \mathbf{X}, \mathbf{F}\right] \\
-N^{-1 / 2} \sum_{i=1}^{[r N]} p_{i}\left(0 \mid X_{i}, f_{t}\right) G_{i}^{0}\left(X_{i}\right) G_{i}^{0}\left(X_{i}\right)^{\top}\left(f_{t}^{0}-f_{t}\right) \|=o_{p}(1) .
\end{gathered}
$$

Hence, by (A.39), (A.40) and (A.41), we have

$$
\begin{aligned}
N^{-1 / 2} \widehat{S}_{[r N] t} & =N^{-1 / 2} \sum_{i=1}^{[r N]} G_{i}^{0}\left(X_{i}\right)\left(\tau-I\left(\varepsilon_{i t}<0\right)\right) \\
& -N^{-1 / 2} \sum_{i=1}^{[r N]} p_{i}\left(0 \mid X_{i}, f_{t}\right) G_{i}^{0}\left(X_{i}\right) G_{i}^{0}\left(X_{i}\right)^{\top}\left(\widehat{f}_{t}-f_{t}^{0}\right)+o_{p}(1) .
\end{aligned}
$$

This result, together with (A.37), implies

$$
N^{-1 / 2} \widehat{S}_{[r N] t}=\digamma_{N t}(r)-\Lambda_{N t}(r)\left\{\Lambda_{N t}(1)\right\}^{-1} \digamma_{N t}(1)+o_{p}(1)
$$

Thus, $N^{-1 / 2} \widehat{S}_{N t}=o_{p}(1)$. By following the argument above again, we have $\| N^{-1 / 2} \sum_{j=1}^{N} \widehat{v}_{j t} K_{j N}^{*}-$ $N^{-1 / 2} \sum_{j=1}^{N} v_{j t} K_{j N}^{*} \|=O_{p}(1)$. Also $\left\|N^{-1 / 2} \sum_{j=1}^{N} v_{j t} K_{j N}^{*}\right\|=O_{p}(1)$ by the weak law of large numbers. Hence, $\left\|N^{-1 / 2} \sum_{j=1}^{N} \widehat{v}_{j t} K_{j N}^{*}\right\|=O_{p}(1)$. Therefore

$$
N^{-1} \sum_{j=1}^{N} \widehat{v}_{j t} K_{j N}^{*} \widehat{S}_{N}^{\top}=O_{p}(1) o_{p}(1)=o_{p}(1) .
$$

By (A.37) and (A.38), $\widehat{w}_{N t}=o_{p}(1)$. By this result and also applying the identity that $\sum_{l=1}^{N} a_{l} b_{l}=\left(\sum_{l=1}^{N-1}\left(a_{l}-a_{l+1}\right) \sum_{j=1}^{l} b_{j}\right)+a_{N} \sum_{l=1}^{N} b_{l}$ to $\sum_{j=1}^{N} K_{i j}^{*} \widehat{v}_{j}^{\top}$ and then again to the sum over $i$, we obtain

$$
\begin{aligned}
\widehat{\Omega}_{N t, M=b N} & =N^{-1} \sum_{i=1}^{N-1} N^{-1} \sum_{j=1}^{N-1} N^{2}\left(\left(K_{i j}^{*}-K_{i, j+1}^{*}\right)-\left(K_{i+1, j}^{*}-K_{i+1, j+1}^{*}\right)\right) N^{-1 / 2} \widehat{S}_{i t} N^{-1 / 2} \widehat{S}_{j t}^{\top} \\
& +N^{-1} \sum_{j=1}^{N} \widehat{v}_{j t} K_{j N}^{*} \widehat{S}_{N t}^{\top}+o_{p}(1),
\end{aligned}
$$

and thus

$$
\begin{aligned}
& \widehat{\Omega}_{N t, M=b N}=\sum_{i=1}^{N-1} \sum_{j=1}^{N-1}\left(\left(K_{i j}^{*}-K_{i, j+1}^{*}\right)-\left(K_{i+1, j}^{*}-K_{i+1, j+1}^{*}\right)\right) \frac{\widehat{S}_{i t}}{\sqrt{N}} \frac{\widehat{S}_{j t}^{\top}}{\sqrt{N}} \\
& +o_{p}(1) .
\end{aligned}
$$


Moreover,

$$
N^{2}\left(\left(K_{i j}^{*}-K_{i, j+1}^{*}\right)-\left(K_{i+1, j}^{*}-K_{i+1, j+1}^{*}\right)\right)=-D_{b N}\{(i-j) / N\} .
$$

Also $\lim _{N \rightarrow \infty} D_{b N}(r)=\frac{1}{b^{2}} K^{* \prime \prime}\left(\frac{r}{b}\right),\left\|\Lambda_{N t}(r)-r \Lambda_{t}^{0}\right\|=o_{p}(1)$, where $\Lambda_{t}^{0}=\lim _{N \rightarrow \infty} \Lambda_{N t}^{0}$ and $\digamma_{N t}(r) \stackrel{\mathcal{D}}{\rightarrow} W_{J+1}(r) \Upsilon^{\top}$. Thus,

$$
\left(\Lambda_{N t}(r), \digamma_{N t}(r)^{\top}, D_{b N}(r)\right) \stackrel{\mathcal{D}}{\rightarrow}\left(r \Lambda_{t}^{0}, \Upsilon W_{J+1}(r)^{\top}, \frac{1}{b^{2}} K^{* \prime \prime}\left(\frac{r}{b}\right)\right) .
$$

Hence, by (A.42), (A.43), and (A.44), it follows that

$$
\begin{aligned}
\widehat{\Omega}_{N t, M=b N} & =\int_{0}^{1} \int_{0}^{1}-D_{b N}(r-s)\left[\digamma_{N t}(r)-\Lambda_{N t}(r)\left\{\Lambda_{N t}(1)\right\}^{-1} \digamma_{N t}(1)\right] \\
& \times\left[\digamma_{N t}(s)-\Lambda_{N t}(s)\left\{\Lambda_{N t}(1)\right\}^{-1} \digamma_{N t}(1)\right]^{\top} d r d s+o_{p}(1) .
\end{aligned}
$$

By the continuous mapping theorem,

$\widehat{\Omega}_{N, M=b N} \stackrel{\mathcal{D}}{\rightarrow} \Upsilon \int_{0}^{1} \int_{0}^{1}-\frac{1}{b^{2}} K^{* \prime \prime}\left(\frac{r-s}{b}\right)\left\{W_{J+1}(r)-r W_{J+1}(1)\right\}\left\{W_{J+1}(s)-s W_{J+1}(1)\right\}^{\top} d r d s \Upsilon^{\top}$.

Then the proof is completed.

\subsection{Proofs of Theorems 5 and 6}

Proof. By (A.37), $\widehat{f}_{t}-f_{t}^{0}=N^{-1 / 2} \Lambda_{N t}(1)^{-1} \digamma_{N t}(1)+o_{p}\left(N^{-1 / 2}\right)$. Then under $H_{0}$, we have

$$
N^{1 / 2}\left(R \widehat{f}_{t}-r\right)=R \Lambda_{N t}(1)^{-1} \digamma_{N t}(1)+o_{p}(1)
$$

It directly follows from (A.45), (A.46) and (A.47) that

$$
\begin{aligned}
& F_{N t, b} \stackrel{\mathcal{D}}{\rightarrow}\left\{R \Lambda_{t}^{0-1} \Upsilon W_{J+1}(1)\right\}^{\top}\left\{R \tau(1-\tau) \Lambda_{t}^{0-1}\right. \\
& \left.\times\left(\Upsilon \int_{0}^{1} \int_{0}^{1}-\frac{1}{b^{2}} K^{* \prime \prime}\left(\frac{r-s}{b}\right) B_{J+1}(r) B_{J+1}(s)^{\top} d r d s \Upsilon^{\top}\right) \Lambda_{t}^{0-1} R^{\top}\right\}^{-1} \\
& \times R \Lambda_{t}^{0-1} \Upsilon W_{J+1}(1) / q .
\end{aligned}
$$

Since $R \Lambda_{t}^{0-1} \Upsilon W_{J+1}(1)$ is a $q \times 1$ vector of normal random variables with mean zero and variance $R \Lambda_{t}^{0-1} \Upsilon \Upsilon^{\top} \Lambda_{t}^{0-1} R^{\top}, R \Lambda_{t}^{0-1} \Upsilon W_{J+1}(1)$ can be written as $\Upsilon_{t}^{*} W_{q}(1)$, where $\Upsilon_{t}^{*} \Upsilon_{t}^{* \top}=$ $R \Lambda_{t}^{0-1} \Upsilon \Upsilon^{\top} \Lambda_{t}^{0-1} R^{\top}$. Then replacing $R \Lambda_{t}^{0-1} \Upsilon W_{J+1}(1)$ by $\Upsilon_{t}^{*} W_{q}(1)$ and canceling $\Upsilon_{t}^{*}$ in the above equation, we have the result in Theorem 5. Moreover, under the alternative that $H_{1}$ : $R f_{t}^{0}=r+c N^{-1 / 2}$, we have

$$
\begin{aligned}
N^{1 / 2}\left(R \widehat{f}_{t}-r\right) & =N^{1 / 2}\left(R f_{t}^{0}-r\right)+R \Lambda_{N t}(1)^{-1} \digamma_{N t}(1)+o_{p}(1) \\
& =c+R \Lambda_{N t}(1)^{-1} \digamma_{N t}(1)+o_{p}(1) .
\end{aligned}
$$


Thus by (A.45), we have

$$
\begin{aligned}
& F_{N t, b} \stackrel{\mathcal{D}}{\rightarrow}\left\{c+R \Lambda_{t}^{0-1} \Upsilon W_{J+1}(1)\right\}^{\top}\left\{R \tau(1-\tau) \Lambda_{t}^{0-1}\right. \\
& \left.\times\left(\Upsilon \int_{0}^{1} \int_{0}^{1}-\frac{1}{b^{2}} K^{* \prime \prime}\left(\frac{r-s}{b}\right) B_{J+1}(r) B_{J+1}(s)^{\top} d r d s \Upsilon^{\top}\right) \Lambda_{t}^{0-1} R^{\top}\right\}^{-1} \\
& \times\left\{c+R \Lambda_{t}^{0-1} \Upsilon W_{J+1}(1)\right\} / q .
\end{aligned}
$$

Also $c+R \Lambda_{t}^{0-1} \Upsilon W_{J+1}(1) \equiv c+\Upsilon_{t}^{*} W_{q}(1)=\Upsilon_{t}^{*}\left(\Upsilon_{t}^{*-1} c+W_{q}(1)\right)$. Then the result in Theorem 6 follows from the above results. The proof is completed.

\section{References}

[1] Angrist, J., and Pischke, J. (2009): "Mostly Harmless Econometrics: An Empiricist's Companion," 1st Edition, New Jersey: Princeton University Press.

[2] Babu, G. J. (1989): "Strong Representations for LAD Estimators in Linear Models," Probability Theory and Related Fields, 83, 547-558.

[3] Bai, J. and Li, K. (2012): "Statistical Analysis of Factor Models of High Dimension," The Annals of Statistics, 40,436-465.

[4] Bai, J. and Ng, S. (2002): "Determining the Number of Factors in Approximate Factor Models," Econometrica, 70,191-221.

[5] Banz, R.W. (1981): "The Relationship between Return and Market Value of Common Stocks," Journal of Financial Economics, 9, 3-18.

[6] Bassett., G.W., Koenker, R. and Kordas, G. (2004): "Pessimistic Portfolio Allocation and Choquet Expected Utility," Journal of Financial Econometrics, 2, 477-492.

[7] Basu, S. (1977): "The Investment Performance of Common Stocks in Relation to Their Price to Earnings Ratio: a Test of the Efficient Markets Hypothesis," Journal of Finance, 32, 663-682.

[8] Bellman, R. E. (1961): "Adaptive Control Processes," Princeton University Press, Princeton, N.J..

[9] Belloni, A., Chernozhukov, V., and Fernandez-Val, I. (2016): "Conditional Quantile Processes based on Series or Many Regressors," Available at arXiv:1105.6154v1.

[10] Boneva, L., Linton, O. and Vogt, M. (2015): "The Effect of Fragmentation in Trading on Market Quality in the UK Equity Market," Journal of Applied Econometrics, Available at http://dx.doi.org/10.1002/jae.2438

[11] Bosq, D. (1998): "Nonparametric Statistics for Stochastic Processes," New York: Springer-Verlag. 
[12] Brown, S. J. (1989): "The Number of Factors in Security Returns," Journal of Finance, 44, 1247-1262.

[13] Bryzgalova, S. (2015): "Spurious Factors in Linear Asset Pricing Models. Working Paper.

[14] Cai, T. and Liu, W. (2011): "Adaptive thresholding for sparse covariance matrix estimation," Journal of the American Statistical Association, 106, 672-684.

[15] Chen, X. (2011): "Penalized Sieve Estimation and Inference of Semi-Nonparametric Dynamic Models: A Selective Review," Cowles Foundation Discussion Paper No. 1804.

[16] Chen, X., and Christensen, T. (2015): "Optimal Uniform Convergence Rates and Asymptotic Normality for Series Estimators under Weak Dependence and Weak Conditions," Journal of Econometrics, $188,447-465$.

[17] Chen, X., and Liao, Z. (2012): "Asymptotic Properties of Penalized M Estimators with Time Series Observations," In Causality, Prediction and Specification Analysis: Essays in Honour of Halbert White (eds. X. Chen and N. Swanson). Springer-Verlag, Berlin.

[18] Chen, X., and Pouzo, D. (2015): "Sieve Quasi-Likelihood Ratio Inference on Semi-Nonparametric Conditional Moment Models," Econometrica, 83, 1013-1079.

[19] Conley, T. G. (1999): "GMM Estimation with Cross-Sectional Dependence," Journal of Econometrics, $92,1-45$.

[20] Connor, G., and Korajczyk, R.A. (1993): "A Test for the Number of Factors in an Approximate Factor Model," Journal of Finance, 48, 1263-1288.

[21] Connor, G., and Linton, O. (2007): "Semiparametric Estimation of a Characteristic-Based Factor Model of Stock Returns," Journal of Empirical Finance, 14, 694-717.

[22] Connor, G., Hagmann, M., and Linton, O. (2012): "Efficient Semiparametric Estimation of the FamaFrench Model and Extensions," Econometrica, 80, 713-754.

[23] Daniel, K., and Titman, S. (1997): "Evidence on the Characteristics of Cross-sectional Variation in Stock Returns," Journal of Finance, 52, 1-34.

[24] Davis, J. (1994): “The Cross-Section of Realized Stock Returns: the pre-Compustat Evidence," Journal of Finance, 49, 1579-1593.

[25] de Boor, C. (2001): A Practical Guide to Splines," Applied Mathematical Sciences 27, New York: Springer.

[26] Demko, S. (1986): "Spectral Bounds for $\left|a^{-1}\right|_{\infty}$, " Journal of Approximation Theory, 48, 207-212. 
[27] Dong, C., Gao, J. and Peng, B. (2015): "Semiparametric Single-Index Panel Data Models with CrossSectional Dependence," Journal of Econometrics, 188, 301-312

[28] Fama, E.F., and French, K.R. (1992): "The Cross-Section of Expected Stock Returns," Journal of Finance, 47, 427-465.

[29] Fama, E.F., and French, K.R. (1993): "Common Risk Factors in the Returns to Stocks and Bonds," Journal of Financial Economics, 33, 3-56.

[30] Fama, E.F., and French, K.R. (1995): "Size and Book to Market Factors in Earnings and Returns," Journal of Finance, 50, 131-156.

[31] Fama, E.F., and French, K.R. (1996): "Multifactor Explanations of Asset Pricing Anomalies," Journal of Finance, 51, 55-84.

[32] Fama, E.F., and French, K.R. (1998): "Value versus Growth: the International Evidence," Journal of Finance, 53, 1975-2000.

[33] Fan, J., Liao, Y. and, Micheva, M. (2013): "Large Covariance Estimation by Thresholding Principal Orthogonal Complements (with Discussion)," Journal of the Royal Statistical Society Series B, 75, 603-680.

[34] Fan, J., Liao, Y. and Wang, W. (2016): "Projected Principal Component Analysis in Factor Models," The Annals of Statistics, 44, 219-254.

[35] Gao, J., Lu, Z., and Tjøstheim, D. (2006): "Estimation in Semiparametric Spatial Regression," The Annals of Statistics, 34, 1395-1435.

[36] Haugen, R. (1995): "The New Finance: the case against Efficient Markets," New Jersey: Prentice-Hall, Englewood Cliffs.

[37] He, X. and Shi, P. (1996): "Bivariate tensor-product B-splines in a partly linear model," Journal of Multivariate Analysis, 58, 162-181.

[38] Hodrick, R., Ng, D., and Sengmueller, P. (1999): "An International Dynamic Asset Pricing Model," International Taxation and Public Finance, 6, 597-620.

[39] Horowitz, J. L., and Lee, S. (2005): "Nonparametric Estimation of an Additive Quantile Regression Model," Journal of the American Statistical Association, 100, 1238-1249.

[40] Horowitz, J. L., and Mammen, E. (2011): "Oracle-Efficient Nonparametric Estimation of an Additive Model with an Unknown Link Function," Econometric Theory, 27, 582-608. 
[41] Kiefer, N. M., and Vogelsang, T. J. (2002): "Heteroskedasticity-Autocorrelation Robust Standard Errors using the Bartlett Kernel without Truncation," Econometrica, 70, 2093-2095.

[42] Kiefer, N. M., and Vogelsang, T. J. (2005): "A New Asymptotic Theory for HeteroskedasticityAutocorrelation Robust Tests," Econometric Theory, 21, 1130-1164.

[43] Knight, K. (1998): "Limiting Distribution for $\mathrm{L}_{1}$ Regression Estimators under General Conditions," The Annals of Statistics, 26, 755-770.

[44] Koenker, R., and Bassett, G. (1978): "Regression Quantiles," Econometrica, 46, 33-50.

[45] Lakonishok, J., Shleifer, A., and Vishny, R.W. (1994): "Contrarian Investment, Extrapolation and Risk," Journal of Finance, 49, 1541-1578.

[46] Lam, C. and Yao, Q. (2012): "Factor Modeling for High-dimensional Time Series: Inference for the Number of Factors," The Annals of Statistics, 40, 694-726.

[47] Lee, J., and Robinson, P. M. (2016): "Series Estimation under Cross-Sectional Dependence," Journal of Econometrics, 190, 1-17.

[48] Lewellen, J. (1999): "The Time-Series Relations among Expected Return, Risk, and Book to Market Value," Journal of Financial Economics, 54, 5-44.

[49] Li, Q., Cheng, G., Fan, J., and Wang, Y. (2017): "Embracing Blessing of Dimensionality in Factor Models," Journal of American Statistical Association, forthcoming.

[50] Ma, S., Song, Q., and Wang, L. (2013): "Simultaneous Variable Selection and Estimation in Semiparametric Modelling of Longitudinal/clustered Data," Bernoulli, 19, 252-274.

[51] Ma, S., and Yang, L. (2011): "Spline-Backfitted Kernel Smoothing of Partially Linear Additive Model," Journal of Statistical Planning and Inference, 14, 204-219.

[52] MacKinlay, A.C. (1995): "Multifactor Models do not Explain Deviations from the CAPM," Journal of Finance, 38, 3-23.

[53] Merlevède, Peligrad, F., M., and Rio, E. (2009): "Bernstein Inequality and Moderate Deviations under Strong Mixing Conditions," IMS Collections, High Dimensional Probability V: The Luminy Volume, 5, 273-292.

[54] Pesaran, M.H. (2006): "Estimation and Inference in Large Heterogeneous Panels with a Multifactor Error Structure," Econometrica, 74, 967-1012. 
[55] Powell, J. L. (1991): "Estimation of Monotonic Regression Models under Quantile Restrictions," In W. Barnett, J. Powell, G. Tauchen (Eds.), Nonparametric and Semiparametric Models in Econometrics, Cambridge: Cambridge University Press.

[56] Robinson, P.M., and Thawornkaiwong, S. (2012): "Statistical Inference on Regression with Spatial Dependence," Journal of Econometrics, 167, 521-542.

[57] Rosenberg, B. (1974): Extra-Market Components of Covariance Among Security Prices," Journal of Financial and Quantitative Analysis, 9, 263-274.

[58] Rosenberg, B., Reid, K., and Lanstein, R. (1985): "Persuasive Evidence of Market Inefficiency," Journal of Portfolio Management, 11, 9-17.

\section{Supplementary Material}

In this supplement, we present Lemmas $\mathbf{S . 1}$ S.3 which are used to prove Lemma 2 in Section 9.1. We also give Lemmas S.4 S.6 which are used in the proofs of Lemmas 3 and 4, and Lemmas S.7 $\mathrm{S.8}$ which are used in the proofs of Lemma 5 in Section 9.2 .

Lemma S.1. Under Conditions (C1) and (C2), and $K_{N}^{2} N^{-1}(\log N T)^{2}(\log N)^{8}=o(1)$ and $K_{N}^{-1}=o(1)$,

$$
\begin{aligned}
& \sup _{1 \leq t \leq T} \sup _{\left\|\boldsymbol{\vartheta}_{t}-\boldsymbol{\vartheta}_{t}^{0}\right\| \leq C K_{N}^{1 / 2} N^{-1 / 2}}\left\|N^{-1} \sum_{i=1}^{N} \widetilde{G}_{t N, i}\left(\boldsymbol{\vartheta}_{t}\right)-N^{-1} \sum_{i=1}^{N} \widetilde{G}_{t N, i}\left(\boldsymbol{\vartheta}_{t}^{0}\right)\right\| \\
& =O_{p}\left(K_{N}^{3 / 2} N^{-3 / 4} \sqrt{\log N T}\right) .
\end{aligned}
$$

Proof. Let $B_{N}=\left\{\boldsymbol{\vartheta}_{t}:\left\|\boldsymbol{\vartheta}_{t}-\boldsymbol{\vartheta}_{t}^{0}\right\| \leq C K_{N}^{1 / 2} N^{-1 / 2}\right\}$. By taking the same strategy as given in Lemma A.5 of Horowitz and Lee (2005), we cover the ball $B_{N}$ with cubes $\mathcal{C}=\left\{\mathcal{C}\left(\boldsymbol{\vartheta}_{t, v}\right)\right\}$, where $\mathcal{C}\left(\boldsymbol{\vartheta}_{t, v}\right)$ is a cube containing $\left(\boldsymbol{\vartheta}_{t, v}-\boldsymbol{\vartheta}_{t}^{0}\right)$ with sides of $C\left\{d(N) / N^{5}\right\}^{1 / 2}$ such that $\boldsymbol{\vartheta}_{t, v} \in$ $B_{N}$. Then the number of the cubes covering the ball $B_{N}$ is $V=\left(2 N^{2}\right)^{d(N)}$. Moreover, we have $\left\|\left(\boldsymbol{\vartheta}_{t}-\boldsymbol{\vartheta}_{t}^{0}\right)-\left(\boldsymbol{\vartheta}_{t, v}-\boldsymbol{\vartheta}_{t}^{0}\right)\right\| \leq C\left\{d(N) / N^{5 / 2}\right\}$ for any $\boldsymbol{\vartheta}_{t}-\boldsymbol{\vartheta}_{t}^{0} \in \mathcal{C}\left(\boldsymbol{\vartheta}_{t, v}\right)$, where $v=1, \ldots, V$. First we can decompose

$$
\begin{aligned}
& \sup _{\boldsymbol{\vartheta}_{t} \in B_{N}}\left\|N^{-1} \sum_{i=1}^{N} \widetilde{G}_{t N, i}\left(\boldsymbol{\vartheta}_{t}\right)-N^{-1} \sum_{i=1}^{N} \widetilde{G}_{t N, i}\left(\boldsymbol{\vartheta}_{t}^{0}\right)\right\| \\
& \leq \max _{1 \leq v \leq V} \sup _{\left(\boldsymbol{\vartheta}_{t}-\boldsymbol{\vartheta}_{t}^{0}\right) \in \mathcal{C}\left(\boldsymbol{\vartheta}_{t, v}\right)}\left\|N^{-1} \sum_{i=1}^{N} \widetilde{G}_{t N, i}\left(\boldsymbol{\vartheta}_{t}\right)-N^{-1} \sum_{i=1}^{N} \widetilde{G}_{t N, i}\left(\boldsymbol{\vartheta}_{t, v}\right)\right\| \\
& +\max _{1 \leq v \leq V}\left\|N^{-1} \sum_{i=1}^{N} \widetilde{G}_{t N, i}\left(\boldsymbol{\vartheta}_{t, v}\right)-N^{-1} \sum_{i=1}^{N} \widetilde{G}_{t N, i}\left(\boldsymbol{\vartheta}_{t}^{0}\right)\right\| \\
& =\Delta_{t N, 1}+\Delta_{t N, 2}
\end{aligned}
$$


Let $\gamma_{N}=C\left\{d(N) / n^{5 / 2}\right\}$. By the same argument as given in the proof of Lemma A.5 in Horowitz and Lee (2005), we have

$$
\Delta_{t N, 1} \leq \max _{1 \leq v \leq V}\left|\Gamma_{t N, 1 v}\right|+\max _{1 \leq v \leq V}\left|\Gamma_{t N, 2 v}\right|
$$

where

$$
\begin{aligned}
\Gamma_{t N, 1 v} & =N^{-1} \sum_{i=1}^{N}\left\|Z_{i}\right\|\left[F_{i}\left[Z_{i}^{\top}\left(\boldsymbol{\vartheta}_{t, v}-\boldsymbol{\vartheta}_{t}^{0}\right)-b_{t}\left(X_{i}\right)+\left\|Z_{i}\right\| \gamma_{N} \mid X_{i}, f_{t}\right]\right. \\
& \left.-F_{i}\left[Z_{i}^{\top}\left(\boldsymbol{\vartheta}_{t, v}-\boldsymbol{\vartheta}_{t}^{0}\right)-b_{t}\left(X_{i}\right)-\left\|Z_{i}\right\| \gamma_{N} \mid X_{i}, f_{t}\right]\right], \\
\Gamma_{t N, 2 v} & =N^{-1} \sum_{i=1}^{N} \Gamma_{t N, 2 v, i}=N^{-1} \sum_{i=1}^{N}\left\|Z_{i}\right\|\left[\left[I\left\{\varepsilon_{i t} \leq Z_{i}^{\top}\left(\boldsymbol{\vartheta}_{t, v}-\boldsymbol{\vartheta}_{t}^{0}\right)-b_{t}\left(X_{i}\right)+\left\|Z_{i}\right\| \gamma_{N}\right\}\right.\right. \\
& \left.-F_{i}\left\{Z_{i}^{\top}\left(\boldsymbol{\vartheta}_{t, v}-\boldsymbol{\vartheta}_{t}^{0}\right)-b_{t}\left(X_{i}\right)+\left\|Z_{i}\right\| \gamma_{N} \mid X_{i}, f_{t}\right\}\right] \\
& \left.-\left[I\left\{\varepsilon_{i t} \leq Z_{i}^{\top}\left(\boldsymbol{\vartheta}_{t, v}-\boldsymbol{\vartheta}_{t}^{0}\right)-b_{t}\left(X_{i}\right)\right\}-F_{i}\left\{Z_{i}^{\top}\left(\boldsymbol{\vartheta}_{t, v}-\boldsymbol{\vartheta}_{t}^{0}\right)-b_{t}\left(X_{i}\right) \mid X_{i}, f_{t}\right\}\right]\right] .
\end{aligned}
$$

By Condition (C2), we have that there are some constants $0<c^{\prime}, c^{\prime \prime}<\infty$ such that

$$
\sup _{1 \leq t \leq T} \max _{1 \leq v \leq V}\left|\Gamma_{t N, 1 v}\right| \leq c^{\prime} \gamma_{N} \max _{1 \leq i \leq N}\left\|Z_{i} \mid\right\|\left\|Z_{i}\right\| \leq c^{\prime \prime}\left\{d(N) / N^{5 / 2}\right\} K_{N}=O\left(K_{N}^{2} N^{-5 / 2}\right) .
$$

Next we will show the convergence rate for $\max _{1 \leq v \leq V}\left|\Gamma_{t N, 2 v}\right|$. It is easy to see that $E\left(\Gamma_{t N, 2 v, i}\right)=$ 0. Also $\left|\Gamma_{t N, 2 v, i}\right| \leq 4\left\|Z_{i}\right\| \leq c_{1} K_{N}^{1 / 2}$ for some constant $0<c_{1}<\infty$. Moreover,

$$
\begin{aligned}
& E\left[\left\|Z_{i}\right\| I\left\{\varepsilon_{i t} \leq Z_{i}^{\top}\left(\boldsymbol{\vartheta}_{t, v}-\boldsymbol{\vartheta}_{t}^{0}\right)-b_{t}\left(X_{i}\right)+\left\|Z_{i}\right\| \gamma_{N}\right\}-I\left\{\varepsilon_{i t} \leq Z_{i}^{\top}\left(\boldsymbol{\vartheta}_{t, v}-\boldsymbol{\vartheta}_{t}^{0}\right)-b_{t}\left(X_{i}\right)\right\}\right]^{2} \\
& \asymp E\left\{\left\|Z_{i}\right\|^{2}\left\|Z_{i}\right\| \gamma_{N}\right\} \leq c_{2}^{*} \gamma_{N} K_{N}^{1 / 2} \leq c_{2} K_{N}^{3 / 2} N^{-5 / 2},
\end{aligned}
$$

for some constants $0<c_{2}^{*}<c_{2}<\infty$. Hence $E\left(\Gamma_{t N, 2 v, i}\right)^{2} \leq c_{2} K_{N}^{3 / 2} N^{-5 / 2}$. By Condition (C1), we have for $i \neq j$,

$$
\left|E\left(\Gamma_{t N, 2 v, i} \Gamma_{t N, 2 v, j}\right)\right| \leq 2 \phi(|j-i|)^{1 / 2}\left\{E\left(\Gamma_{t N, 2 v, i}^{2}\right) E\left(\Gamma_{t N, 2 v, j}^{2}\right)\right\}^{1 / 2} \leq 2 c_{2} \phi(|j-i|)^{1 / 2} K_{N}^{3 / 2} N^{-5 / 2} .
$$

Hence

$$
\begin{aligned}
& E\left(\Gamma_{t N, 2 v, i}\right)^{2}+2 \sum_{j>i}\left|E\left(\Gamma_{t N, 2 v, i} \Gamma_{t N, 2 v, j}\right)\right| \\
& \leq c_{2} K_{N}^{3 / 2} N^{-5 / 2}+4 c_{2} \sum_{k=1}^{N} K_{1} e^{-\lambda_{1} k / 2} K_{N}^{3 / 2} N^{-5 / 2} \\
& \leq c_{2} K_{N}^{3 / 2} N^{-5 / 2}\left(1+4 K_{1}\left(1-e^{-\lambda_{1} / 2}\right)^{-1}\right)=c_{3} K_{N}^{3 / 2} N^{-5 / 2}
\end{aligned}
$$

where $c_{3}=c_{2}\left(1+4 K_{1}\left(1-e^{-\lambda_{1} / 2}\right)^{-1}\right)$. By Condition $(\mathrm{C} 1)$, for each fixed $t$, the sequence $\left\{\left(X_{i}, f_{t}, \varepsilon_{i t}\right), 1 \leq i \leq N\right\}$ has the $\phi$-mixing coefficient $\phi(k) \leq K_{1} e^{-\lambda_{1} k}$ for $K_{1}, \lambda_{1}>0$. Thus, by the Bernstein's inequality given in Lemma 1, we have for $N$ sufficiently large,

$$
\begin{aligned}
& P\left(\left|\Gamma_{t N, 2 v}\right| \geq a K_{N}^{3 / 2} N^{-1}(\log N T)^{3}\right) \\
& \leq \exp \left(-\frac{C_{1}\left(a K_{N}^{3 / 2}(\log N T)^{3}\right)^{2}}{c_{3} K_{N}^{3 / 2} N^{-5 / 2} N+c_{1}^{2} K_{N}+a K_{N}^{3 / 2}(\log N T)^{3} c_{1} K_{N}^{1 / 2} \log (N)^{2}}\right) \leq(N T)^{-c_{4} a^{2} K_{N}}
\end{aligned}
$$


for some constant $0<c_{4}<\infty$. By the union bound of probability, we have

$$
\begin{aligned}
& P\left(\sup _{1 \leq t \leq T} \max _{1 \leq v \leq V}\left|\Gamma_{t N, 2 v}\right| \geq a K_{N}^{3 / 2} N^{-1}(\log N T)^{3}\right) \\
& \leq\left(2 N^{2}\right)^{d(N)} T(N T)^{-c_{4} a^{2} K_{N}} \leq 2^{d(N)} N^{2\left(1+J K_{N}\right)-c_{4} a^{2} K_{N}} T^{1-c_{4} a^{2} K_{N}} .
\end{aligned}
$$

Hence, taking a large enough, one has

$$
P\left(\sup _{1 \leq t \leq T} \max _{1 \leq v \leq V}\left|\Gamma_{t N, 2 v}\right| \geq a K_{N}^{3 / 2} N^{-1}(\log N)^{3}\right) \leq 2^{K_{N}} N^{-K_{N}} T^{-K_{N}}
$$

Then we have

$$
\sup _{1 \leq t \leq T} \max _{1 \leq v \leq V}\left|\Gamma_{t N, 2 v}\right|=O_{p}\left\{K_{N}^{3 / 2} N^{-1}(\log N T)^{3}\right\} .
$$

Next we will show the convergence rate for $\Delta_{t N, 2}$. Let $\widetilde{g}_{t N, i, \ell}\left(\boldsymbol{\vartheta}_{t, v}\right)$ be the $\ell^{\text {th }}$ element in $\widetilde{G}_{t N, i}\left(\boldsymbol{\vartheta}_{t, v}\right)-\widetilde{G}_{t N, i}\left(\boldsymbol{\vartheta}_{t}^{0}\right)$ for $\ell=1, \ldots, d(N)$. It is easy to see that $E\left\{\widetilde{g}_{t N, i, \ell}\left(\boldsymbol{\vartheta}_{t, v}\right)\right\}=0$. Also $\left|\widetilde{g}_{t N, i, \ell}\left(\boldsymbol{\vartheta}_{t, v}\right)\right| \leq 4\left|Z_{i \ell}\right| \leq c_{1} K_{N}^{1 / 2}$ for some constant $0<c_{1}<\infty$. Moreover,

$$
\begin{aligned}
& E\left[\left[I\left\{\varepsilon_{i t} \leq Z_{i}^{\top}\left(\boldsymbol{\vartheta}_{t, v}-\boldsymbol{\vartheta}_{t}^{0}\right)-b_{t}\left(X_{i}\right)\right\}-I\left\{\varepsilon_{i t} \leq-b_{t}\left(X_{i}\right)\right\}\right] Z_{i \ell}\right]^{2} \\
& \leq c_{1}^{\prime}\left\|\boldsymbol{\vartheta}_{t, v}-\boldsymbol{\vartheta}_{t}^{0}\right\| K_{N}^{1 / 2} \leq c_{1}^{\prime} C K_{N}^{1 / 2} N^{-1 / 2} K_{N}^{1 / 2}=c_{1}^{\prime} C K_{N} N^{-1 / 2}
\end{aligned}
$$

for some constant $0<c_{1}^{\prime}<\infty$. Hence $E\left(\widetilde{g}_{t N, i, \ell}\left(\boldsymbol{\vartheta}_{t, v}\right)\right)^{2} \leq c_{1}^{\prime} C K_{N} N^{-1 / 2}$. By Condition (C1), we have for $i \neq j$,

$$
\mid E\left(\widetilde{g}_{t N, i, \ell}\left(\boldsymbol{\vartheta}_{t, v}\right) \widetilde{g}_{t N, j, \ell}\left(\boldsymbol{\vartheta}_{t, v}\right) \mid \leq 4 \phi(|j-i|)^{1 / 2}\left\{E\left(\Gamma_{t N, 2 v, i}^{2}\right) E\left(\Gamma_{t N, 2 v, j}^{2}\right)\right\}^{1 / 2}\right.
$$

Hence

$$
\begin{aligned}
& E\left(\widetilde{g}_{t N, i, \ell}\left(\boldsymbol{\vartheta}_{t, v}\right)\right)^{2}+2 \sum_{j>i} \mid E\left(\widetilde{g}_{t N, i, \ell}\left(\boldsymbol{\vartheta}_{t, v}\right) \widetilde{g}_{t N, j, \ell}\left(\boldsymbol{\vartheta}_{t, v}\right) \mid\right. \\
& \leq c_{1}^{\prime} C K_{N} N^{-1 / 2}+4 \sum_{k=1}^{N} K_{1} e^{-\lambda_{1} k / 2} c_{1}^{\prime} C K_{N} N^{-1 / 2} \\
& \leq c_{1}^{\prime} C K_{N} N^{-1 / 2}\left(1+4 K_{1}\left(1-e^{-\lambda_{1} / 2}\right)^{-1}\right)=c_{2} K_{N} N^{-1 / 2}
\end{aligned}
$$

where $c_{2}=c_{1}^{\prime} C\left(1+4 K_{1}\left(1-e^{-\lambda_{1} / 2}\right)^{-1}\right)$. Thus, by the Bernstein's inequality given in Lemma 1 and $K_{N}^{2} N^{-1}(\log N T)^{2}(\log N)^{8}=o(1)$, we have for $N$ sufficiently large,

$$
\begin{aligned}
& P\left(\left|N^{-1} \sum_{i=1}^{N} \tilde{g}_{t N, i, \ell}\left(\boldsymbol{\vartheta}_{t, v}\right)\right| \geq a K_{N} N^{-3 / 4} \sqrt{\log N T}\right) \\
& \leq \exp \left(-\frac{C_{1}\left(a K_{N} N^{1 / 4} \sqrt{\log N T}\right)^{2}}{c_{2} K_{N} N^{-1 / 2} N+c_{1}^{2} K_{N}+a K_{N} N^{1 / 4}(\log N T)^{1 / 2} c_{1} K_{N}^{1 / 2}(\log N)^{2}}\right) \leq(N T)^{-c_{3} a^{2} K_{N}}
\end{aligned}
$$

for some constant $0<c_{3}<\infty$. By the union bound of probability, we have

$$
P\left(\sup _{1 \leq t \leq T} \sup _{1 \leq \ell \leq d(N)}\left|N^{-1} \sum_{i=1}^{N} \widetilde{g}_{t N, i, \ell}\left(\boldsymbol{\vartheta}_{t, v}\right)\right| \geq a K_{N} N^{-3 / 4} \sqrt{\log N T}\right) \leq d(N) T(N T)^{-c_{3} a^{2} K_{N}} .
$$


Hence,

$$
\begin{aligned}
& P\left(\sup _{1 \leq t \leq T}\left\|N^{-1} \sum_{i=1}^{N} \widetilde{G}_{t N, i}\left(\boldsymbol{\vartheta}_{t, v}\right)-N^{-1} \sum_{i=1}^{N} \widetilde{G}_{t N, i}\left(\boldsymbol{\vartheta}_{t}^{0}\right)\right\| \geq a K_{N}^{3 / 2} N^{-3 / 4} \sqrt{\log N T}\right) \\
& \leq d(N) T(N T)^{-c_{3} a^{2} K_{N}} .
\end{aligned}
$$

By the union bound of probability again, we have

$$
P\left(\sup _{1 \leq t \leq T}\left|\Delta_{t N, 2}\right| \geq a K_{N}^{3 / 2} N^{-3 / 4} \sqrt{\log N T}\right) \leq\left(2 N^{2}\right)^{d(N)} d(N) T(N T)^{-c_{3} a^{2} K_{N}} .
$$

Hence, taking a large enough, one has

$$
P\left(\sup _{1 \leq t \leq T}\left|\Delta_{t N, 2}\right| \geq a K_{N}^{3 / 2} N^{-3 / 4} \sqrt{\log N T}\right) \leq 2^{K_{N}} K_{N} N^{-K_{N}} T^{-K_{N}} .
$$

Then we have

$$
\sup _{1 \leq t \leq T}\left|\Delta_{t N, 2}\right|=O_{p}\left\{K_{N}^{3 / 2} N^{-3 / 4} \sqrt{\log N T}\right\} .
$$

Therefore, by (S.1), (S.2), (S.3), (S.4) and (S.5), we have

$$
\begin{aligned}
\sup _{1 \leq t \leq T} & \sup _{\boldsymbol{\vartheta}_{t} \in B_{N}}\left\|N^{-1} \sum_{i=1}^{N} \widetilde{G}_{t N, i}\left(\boldsymbol{\vartheta}_{t}\right)-N^{-1} \sum_{i=1}^{N} \widetilde{G}_{t N, i}\left(\boldsymbol{\vartheta}_{t}^{0}\right)\right\| \\
& =O_{p}\left\{K_{N}^{2} N^{-5 / 2}+K_{N}^{3 / 2} N^{-1}(\log N T)^{3}+K_{N}^{3 / 2} N^{-3 / 4} \sqrt{\log N T}\right\} \\
& =O_{p}\left(K_{N}^{3 / 2} N^{-3 / 4} \sqrt{\log N T}\right) .
\end{aligned}
$$

Lemma S.2. Under Conditions (C1) and (C2), $\sup _{1 \leq t \leq T}\left\|N^{-1} \sum_{i=1}^{N} G_{t N, i}\left(\widetilde{\vartheta}_{t}\right)\right\|=O_{p}\left(K_{N}^{3 / 2} N^{-1}\right)$.

Lemma S.3. Under Conditions (C2) and (C3), as $N \rightarrow \infty$,

$$
\Psi_{N t}^{-1} G_{t N, i}^{*}\left(\boldsymbol{\vartheta}_{t}\right)=-\left(\boldsymbol{\vartheta}_{t}-\boldsymbol{\vartheta}_{t}^{0}\right)+N^{-1} \Psi_{N t}^{-1} \sum_{i=1}^{N} p_{i}\left(0 \mid X_{i}, f_{t}\right) Z_{i} b_{t}\left(X_{i}\right)+R_{N t}^{*}
$$

where $\left\|R_{N t}^{*}\right\| \leq C^{*}\left\{K_{N}^{1 / 2}\left\|\boldsymbol{\vartheta}_{t}-\boldsymbol{\vartheta}_{t}^{0}\right\|^{2}+K_{N}^{1 / 2-2 r}\right\}$ for some constant $0<C^{*}<\infty$, uniformly in $t$.

Proof. The proofs of Lemmas $\$ .2$ and $\$$.3 follow the same procedure as in Lemmas A.4 and A.7 of Horowitz and Lee (2005) by using the results (A.1) and (A.3) which hold uniformly in $t=1, \ldots, T$.

Lemma S.4. Under Conditions (C2) and (C3),

$E\left\{L_{N t}\left(f_{t}, g\right) \mid \mathbf{X}, \mathbf{F}\right\}=-\left(f_{t}-f_{t}^{0}\right)^{\top} E\left(W_{N t, 1} \mid \mathbf{X}, \mathbf{F}\right)+\frac{1}{2}\left(f_{t}-f_{t}^{0}\right)^{\top} \Lambda_{N t}^{0}\left(f_{t}-f_{t}^{0}\right)+o_{p}\left(\left\|f_{t}-f_{t}^{0}\right\|^{2}\right)$, uniformly in $\left\|\boldsymbol{\lambda}_{j}-\boldsymbol{\lambda}_{j}^{0}\right\| \leq \widetilde{C} d_{N T}^{*}$ and $\left\|f_{t}-f_{t}^{0}\right\| \leq \varpi_{N}$, where $W_{N t, 1}$ is defined in A.26 and $g_{j}\left(x_{j}\right)=B_{j}\left(x_{j}\right)^{\top} \boldsymbol{\lambda}_{j}$. 
Proof. By using the identity of Knight (1998) that

$$
\rho_{\tau}(u-v)-\rho_{\tau}(u)=-v \psi_{\tau}(u)+\int_{0}^{v}(I(u \leq s)-I(u \leq 0)) d s,
$$

we have

$$
\begin{aligned}
& \rho_{\tau}\left(y_{i t}-f_{t}^{\top} G_{i}\left(X_{i}\right)\right)-\rho_{\tau}\left(y_{i t}-f_{t}^{0 \top} G_{i}\left(X_{i}\right)\right) \\
& =-\left(f_{t}-f_{t}^{0}\right)^{\top} G_{i}\left(X_{i}\right) \psi_{\tau}\left(y_{i t}-f_{t}^{0 \top} G_{i}\left(X_{i}\right)\right) \\
& +\int_{0}^{\left(f_{t}-f_{t}^{0}\right)^{\top} G_{i}\left(X_{i}\right)}\left(I\left(y_{i t}-f_{t}^{0 \top} G_{i}\left(X_{i}\right) \leq s\right)-I\left(y_{i t}-f_{t}^{0 \top} G_{i}\left(X_{i}\right) \leq 0\right)\right) d s .
\end{aligned}
$$

By Lipschitz continuity of $p_{i}\left(\varepsilon \mid X_{i}, f_{t}\right)$ given in Condition $(\mathrm{C} 2)$ and boundedness of $f_{j t}^{0}$ in Condition (C3), we have

$$
\begin{aligned}
& F_{i}\left\{f_{t}^{0 \top}\left(G_{i}\left(X_{i}\right)-G_{i}^{0}\left(X_{i}\right)\right)+s \mid X_{i}, f_{t}\right\}-F_{i}\left\{f_{t}^{0 \top}\left(G_{i}\left(X_{i}\right)-G_{i}^{0}\left(X_{i}\right)\right) \mid X_{i}, f_{t}\right\} \\
& =s p_{i}\left\{f_{t}^{0 \top}\left(G_{i}\left(X_{i}\right)-G_{i}^{0}\left(X_{i}\right)\right) \mid X_{i}, f_{t}\right\}+o(s),
\end{aligned}
$$

where $o(\cdot)$ holds uniformly in $\left\|\boldsymbol{\lambda}_{j}-\boldsymbol{\lambda}_{j}^{0}\right\| \leq \widetilde{C} d_{N T}^{*}$ and $\left\|f_{t}-f_{t}^{0}\right\| \leq \varpi_{N}$. Then we have $E\left\{L_{N t}\left(f_{t}, g\right) \mid \mathbf{X}, \mathbf{F}\right\}$

$$
\begin{aligned}
& =-\left(f_{t}-f_{t}^{0}\right)^{\top} E\left(W_{N t, 1} \mid \mathbf{X}, \mathbf{F}\right)+N^{-1} \sum_{i=1}^{N} \int_{0}^{\left(f_{t}-f_{t}^{0}\right)^{\top} G_{i}\left(X_{i}\right)}\left[F_{i}\left\{f_{t}^{0 \top}\left(G_{i}\left(X_{i}\right)-G_{i}^{0}\left(X_{i}\right)\right)+s \mid X_{i}, f_{t}\right\}\right. \\
& \left.-F_{i}\left\{f_{t}^{0 \top}\left(G_{i}\left(X_{i}\right)-G_{i}^{0}\left(X_{i}\right)\right) \mid X_{i}, f_{t}\right\}\right] d s \\
& =-\left(f_{t}-f_{t}^{0}\right)^{\top} E\left(W_{N t, 1} \mid \mathbf{X}, \mathbf{F}\right)+N^{-1} \sum_{i=1}^{N} \int_{0}^{\left(f_{t}-f_{t}^{0}\right)^{\top} G_{i}\left(X_{i}\right)}\left[s p_{i}\left\{f_{t}^{0 \top}\left(G_{i}\left(X_{i}\right)-G_{i}^{0}\left(X_{i}\right)\right) \mid X_{i}, f_{t}\right\}\right] d s \\
& +o\left[\left(f_{t}-f_{t}^{0}\right)^{\top}\left\{N^{-1} \sum_{i=1}^{N} G_{i}\left(X_{i}\right) G_{i}\left(X_{i}\right)^{\top}\right\}\left(f_{t}-f_{t}^{0}\right)\right] \\
& =-\left(f_{t}-f_{t}^{0}\right)^{\top} E\left(W_{N t, 1} \mid \mathbf{X}, \mathbf{F}\right)+\frac{1}{2}\left(f_{t}-f_{t}^{0}\right)^{\top} \times \\
& {\left[N^{-1} \sum_{i=1}^{N} p_{i}\left\{f_{t}^{0 \top}\left(G_{i}\left(X_{i}\right)-G_{i}^{0}\left(X_{i}\right)\right) \mid X_{i}, f_{t}\right\} G_{i}\left(X_{i}\right) G_{i}\left(X_{i}\right)^{\top}\right]\left(f_{t}-f_{t}^{0}\right)} \\
& +o\left[\left(f_{t}-f_{t}^{0}\right)^{\top}\left\{N^{-1} \sum_{i=1}^{N} G_{i}\left(X_{i}\right) G_{i}\left(X_{i}\right)^{\top}\right\}\left(f_{t}-f_{t}^{0}\right)\right] .
\end{aligned}
$$

Since $\sup _{x_{j} \in[a, b]}\left|g_{j}\left(x_{j}\right)-g_{j}^{0}\left(x_{j}\right)\right|=o(1)$, then $\sup _{x \in \mathcal{X}}\left|f_{t}^{0 \top}\left(G_{i}(x)-G_{i}^{0}(x)\right)\right|=o(1)$. By similar reasoning to the proof for Theorem 2 in Lee and Robinson (2016), we have $N^{-1} \sum_{i=1}^{N} G_{i}\left(X_{i}\right) G_{i}\left(X_{i}\right)^{\top}=N^{-1} \sum_{i=1}^{N} E\left\{G_{i}\left(X_{i}\right) G_{i}\left(X_{i}\right)^{\top}\right\}+o_{p}(1)$. Hence, by these results, we have the result in Lemma $\mathbf{S . 4}$.

Lemma S.5. Under Conditions (C2) and (C3), we have

$$
W_{N t, 2}\left(f_{t}, g\right)-E\left(W_{N t, 2}\left(f_{t}, g\right) \mid \mathbf{X}, \mathbf{F}\right)=o_{p}\left(\left\|f_{t}-f_{t}^{0}\right\|^{2}+N^{-1}\right)
$$

uniformly in $\left\|\boldsymbol{\lambda}_{j}-\boldsymbol{\lambda}_{j}^{0}\right\| \leq \widetilde{C} d_{N T}^{*}$ and $\left\|f_{t}-f_{t}^{0}\right\| \leq \varpi_{N}$, where $W_{N t, 2}\left(f_{t}, g\right)$ is defined in A.24 and $g_{j}\left(x_{j}\right)=B_{j}\left(x_{j}\right)^{\top} \boldsymbol{\lambda}_{j}$. 
Proof. By (S.6), we have

$$
W_{N t, 2 i}\left(f_{t}, g\right)=\int_{0}^{\left(f_{t}-f_{t}^{0}\right)^{\top} G_{i}\left(X_{i}\right)}\left(I\left(y_{i t}-f_{t}^{0 \top} G_{i}\left(X_{i}\right) \leq s\right)-I\left(y_{i t}-f_{t}^{0 \top} G_{i}\left(X_{i}\right) \leq 0\right)\right) d s,
$$

and thus

$$
\begin{aligned}
E\left(W_{N t, 2 i}\left(f_{t}, g\right) \mid X_{i}, f_{t}\right) & =\int_{0}^{\left(f_{t}-f_{t}^{0}\right)^{\top} G_{i}\left(X_{i}\right)}\left[F_{i}\left\{f_{t}^{0 \top}\left(G_{i}\left(X_{i}\right)-G_{i}^{0}\left(X_{i}\right)\right)+s \mid X_{i}, f_{t}\right\}\right. \\
& \left.-F_{i}\left\{f_{t}^{0 \top}\left(G_{i}\left(X_{i}\right)-G_{i}^{0}\left(X_{i}\right)\right) \mid X_{i}, f_{t}\right\}\right] d s .
\end{aligned}
$$

By following the same reasoning as the proof for (S.7), we have

$$
\sup _{X_{i} \in[a, b]^{J}}\left|E\left(W_{N t, 2 i}\left(f_{t}, g\right) \mid X_{i}, f_{t}\right)-\frac{1}{2}\left(f_{t}-f_{t}^{0}\right)^{\top} p_{i}\left(0 \mid X_{i}, f_{t}\right) G_{i}\left(X_{i}\right) G_{i}\left(X_{i}\right)^{\top}\left(f_{t}-f_{t}^{0}\right)\right|=o_{p}\left(\left\|f_{t}-f_{t}^{0}\right\|^{2}\right) .
$$

Hence with probability approaching 1 , as $N \rightarrow \infty$,

$$
\sup _{X_{i} \in[a, b]^{J}}\left|E\left(W_{N t, 2 i}\left(f_{t}, g\right) \mid X_{i}, f_{t}\right)\right| \leq C_{W}\left\|f_{t}-f_{t}^{0}\right\|^{2}
$$

for some constant $0<C_{W}<\infty$. Moreover,

$$
\begin{aligned}
& E\left\{W_{N t, 2 i}\left(f_{t}, g\right)\right\}^{2} \\
& =E\left[E\left[\left\{\int_{0}^{\left(f_{t}-f_{t}^{0}\right)^{\top} G_{i}\left(X_{i}\right)}\left(I\left(y_{i t}-f_{t}^{0 \top} G_{i}\left(X_{i}\right) \leq s\right)-I\left(y_{i t}-f_{t}^{0 \top} G_{i}\left(X_{i}\right) \leq 0\right)\right) d s\right\}^{2} \mid X_{i}, f_{t}\right]\right] \\
& \leq E\left[E \left[\left|I\left(y_{i t}-f_{t}^{0 \top} G_{i}\left(X_{i}\right) \leq\left(f_{t}-f_{t}^{0}\right)^{\top} G_{i}\left(X_{i}\right)\right)-I\left(y_{i t}-f_{t}^{0 \top} G_{i}\left(X_{i}\right) \leq 0\right)\right|\right.\right. \\
& \left.\left.\times\left\{\left(f_{t}-f_{t}^{0}\right)^{\top} G_{i}\left(X_{i}\right)\right\}^{2} \mid X_{i}, f_{t}\right]\right] \\
& =E\left[E \left[\mid I\left(\varepsilon_{i t} \leq f_{t}^{\top} G_{i}\left(X_{i}\right)-f_{t}^{0 \top} G_{i}\left(X_{i}\right)^{0}\right)-I\left(\varepsilon_{i t} \leq f_{t}^{0 \top}\left(G_{i}\left(X_{i}\right)-G_{i}\left(X_{i}\right)^{0}\right) \mid\right.\right.\right. \\
& \left.\left.\times\left\{\left(f_{t}-f_{t}^{0}\right)^{\top} G_{i}\left(X_{i}\right)\right\}^{2} \mid X_{i}, f_{t}\right]\right] \\
& \leq C^{\prime \prime} E\left|\left(f_{t}-f_{t}^{0}\right)^{\top} G_{i}\left(X_{i}\right)\right|^{3} \leq C^{\prime \prime \prime} E\left\|f_{t}-f_{t}^{0}\right\|^{3}
\end{aligned}
$$

for some constants $0<C^{\prime \prime}<\infty$ and $0<C^{\prime \prime \prime}<\infty$. Therefore, for $N \rightarrow \infty$,

$$
\begin{aligned}
& E\left\{W_{N t, 2}\left(f_{t}, g\right)-E\left(W_{N t, 2}\left(f_{t}, g\right) \mid \mathbf{X}, \mathbf{F}\right)\right\}^{2} \\
& =N^{-2} \sum_{i=1}^{N} E\left[W_{N t, 2 i}\left(f_{t}, g\right)-E\left(W_{N t, 2 i}\left(f_{t}, g\right) \mid X_{i}, f_{t}\right)\right]^{2} \\
& \leq N^{-2} \sum_{i=1}^{N}\left[2 E\left\{W_{N t, 2 i}\left(f_{t}, g\right)\right\}^{2}+2 E\left[E\left(W_{N t, 2 i}\left(f_{t}, g\right) \mid X_{i}, f_{t}\right)\right]^{2}\right] \\
& \leq N^{-1}\left(2 C^{\prime \prime \prime} E\left\|f_{t}-f_{t}^{0}\right\|^{3}+2 C_{W}^{2} E\left\|f_{t}-f_{t}^{0}\right\|^{4}\right) \leq C^{\prime \prime \prime \prime} N^{-1} E\left\|f_{t}-f_{t}^{0}\right\|^{3},
\end{aligned}
$$

for some constant $0<C^{\prime \prime \prime \prime}<\infty$. Following the same routine procedure as the proof in Lemma $\mathbf{S . 1}$ by applying the Bernstein's inequality, we have

$$
\left.\sup _{\left\|\boldsymbol{\lambda}_{j}-\boldsymbol{\lambda}_{j}^{0}\right\| \leq \widetilde{C} d_{N T}^{*},\left\|f_{t}-f_{t}^{0}\right\| \leq \varpi_{N}}\left\|f_{t}-f_{t}^{0}\right\|\right|^{-3 / 2}\left|W_{N t, 2}\left(f_{t}, g\right)-E\left(W_{N t, 2}\left(f_{t}, g\right) \mid \mathbf{X}, \mathbf{F}\right)\right|=O_{p}\left(N^{-1 / 2}\right) .
$$


Hence, we have $\left|W_{N t, 2}\left(f_{t}, g\right)-E\left(W_{N t, 2}\left(f_{t}, g\right) \mid \mathbf{X}, \mathbf{F}\right)\right|=O_{p}\left(\left\|f_{t}-f_{t}^{0}\right\|^{-3 / 2} N^{-1 / 2}\right)$, uniformly in $\left\|\boldsymbol{\lambda}_{j}-\boldsymbol{\lambda}_{j}^{0}\right\| \leq \widetilde{C} d_{N T}^{*}$ and $\left\|f_{t}-f_{t}^{0}\right\| \leq \varpi_{N}$. Since

$$
\begin{aligned}
N^{-1 / 2}\left\|f_{t}-f_{t}^{0}\right\|^{3 / 2} & \leq\left. N^{-1}\left\|f_{t}-f_{t}^{0}\right\|\right|^{1 / 2}+\left\|f_{t}-f_{t}^{0}\right\|^{2}\left\|f_{t}-f_{t}^{0}\right\|^{1 / 2} \\
& \leq N^{-1} \varpi_{N}+\left\|f_{t}-f_{t}^{0}\right\|^{2} \varpi_{N},
\end{aligned}
$$

then we have $W_{N t, 2}\left(f_{t}, g\right)-E\left(W_{N t, 2}\left(f_{t}, g\right) \mid \mathbf{X}, \mathbf{F}\right)=o_{p}\left(\left\|f_{t}-f_{t}^{0}\right\|^{2}+N^{-1}\right)$, uniformly in $\left\|\boldsymbol{\lambda}_{j}-\boldsymbol{\lambda}_{j}^{0}\right\| \leq \widetilde{C} d_{N T}^{*}$ and $\left\|f_{t}-f_{t}^{0}\right\| \leq \varpi_{N}$.

Lemma S.6. Under Conditions (C1)-(C3), for any there is a stochastically bounded sequence $\delta_{N, j t}$ such that as $N \rightarrow \infty$,

$$
W_{N t, 1}=N^{-1} \sum_{i=1}^{N} G_{i}^{0}\left(X_{i}\right) \psi_{\tau}\left(\varepsilon_{i t}\right)+d_{N T} \delta_{N, t}+o_{p}\left(N^{-1 / 2}\right),
$$

uniformly in $\left\|\boldsymbol{\lambda}_{j}-\boldsymbol{\lambda}_{j}^{0}\right\| \leq \widetilde{C} d_{N T}^{*}$, where $W_{N t, 1}$ is defined in (A.23), $\delta_{N, t}=\left(\delta_{N, j t}, 0 \leq j \leq\right.$ $J)^{\top}$ and $g_{j}\left(x_{j}\right)=B_{j}\left(x_{j}\right)^{\top} \boldsymbol{\lambda}_{j}$.

Proof. Write

$$
W_{N t, 1}=W_{N t, 11}+W_{N t, 12}+W_{N t, 13}
$$

where

$$
\begin{aligned}
W_{N t, 11} & =N^{-1} \sum_{i=1}^{N} G_{i}^{0}\left(X_{i}\right) \psi_{\tau}\left(y_{i t}-f_{t}^{0 \top} G_{i}^{0}\left(X_{i}\right)\right), \\
W_{N t, 12} & =\left(W_{N t j, 12}, 0 \leq j \leq J\right)^{\top}=N^{-1} \sum_{i=1}^{N}\left(G_{i}\left(X_{i}\right)-G_{i}^{0}\left(X_{i}\right)\right) \psi_{\tau}\left(y_{i t}-f_{t}^{0 \top} G_{i}^{0}\left(X_{i}\right)\right), \\
W_{N t, 13} & =\left(W_{N t j, 13}, 0 \leq j \leq J\right)^{\top} \\
& =N^{-1} \sum_{i=1}^{N} G_{i}\left(X_{i}\right)\left\{\psi_{\tau}\left(y_{i t}-f_{t}^{0 \top} G_{i}\left(X_{i}\right)\right)-\psi_{\tau}\left(y_{i t}-f_{t}^{0 \top} G_{i}^{0}\left(X_{i}\right)\right)\right\} .
\end{aligned}
$$

It is easy to see that $E\left(W_{N t j, 12}\right)=0$. Also by the $\phi$-mixing distribution condition given in Condition (C1), we have $\operatorname{var}\left(W_{N t j, 12}\right) \leq C_{W_{12}} N^{-1} d_{N T}^{2}$ for some constant $0<C_{W_{12}}<\infty$, then by following the routine procedure as the proof in Lemma $\mathbf{S . 1}$, we have

$$
\sup _{|| \boldsymbol{\lambda}_{j}-\boldsymbol{\lambda}_{j}^{0} \| \leq \widetilde{C} d_{N T}^{*}}\left|W_{N t j, 12}\right|=o_{p}\left(N^{-1 / 2}\right) .
$$

Moreover,

$$
\begin{aligned}
E\left(W_{N t j, 13} \mid \mathbf{X}, \mathbf{F}\right) & =N^{-1} \sum_{i=1}^{N} g_{j}\left(X_{j i}\right) E\left\{I\left(y_{i t}-f_{t}^{0 \top} G_{i}^{0}\left(X_{i}\right) \leq 0\right)-I\left(y_{i t}-f_{t}^{0 \top} G_{i}\left(X_{i}\right) \leq 0\right) \mid X_{i}, f_{t}\right\} \\
& =N^{-1} \sum_{i=1}^{N} g_{j}\left(X_{j i}\right) \int_{f_{t}^{0 \top}\left(G_{i}\left(X_{i}\right)-G_{i}^{0}\left(X_{i}\right)\right)}^{0} p_{i}\left(s \mid X_{i}, f_{t}\right) d s \\
& =N^{-1} \sum_{i=1}^{N} g_{j}\left(X_{j i}\right) p_{i}\left(0 \mid X_{i}, f_{t}\right) f_{t}^{0 \top}\left(G_{i}^{0}\left(X_{i}\right)-G_{i}\left(X_{i}\right)\right)+O\left(d_{N T}^{2}\right)+o\left(N^{-1}\right) .
\end{aligned}
$$

Let

$$
d_{N T} \delta_{N, j t}=N^{-1} \sum_{i=1}^{N} g_{j}\left(X_{j i}\right) p_{i}\left(0 \mid X_{i}, f_{t}\right) f_{t}^{0 \top}\left(G_{i}^{0}\left(X_{i}\right)-G_{i}\left(X_{i}\right)\right)+O\left(d_{N T}^{2}\right)
$$


Since $N^{-1} \sum_{i=1}^{N}\left\{g_{j}\left(X_{j i}\right)-g_{j}^{0}\left(X_{j i}\right)\right\}^{2} \leq\left(\widetilde{C} d_{N T}^{*}\right)^{2}$, then as $N \rightarrow \infty,\left|d_{N T} \delta_{N, j t}\right| \leq C_{\delta} d_{N T}^{*}$ for some constant $0<C_{\delta}<\infty$. Therefore,

$$
E\left(W_{N t j, 13} \mid \mathbf{X}, \mathbf{F}\right)=d_{N T} \delta_{N, j t}+o\left(N^{-1 / 2}\right)
$$

Also by the $\phi$-mixing condition given in Condition (C1), we have $E\left\{W_{N t j, 13}-E\left(W_{N t j, 13} \mid \mathbf{X}, \mathbf{F}\right)\right\}^{2} \leq$ $C_{\delta}^{\prime} N^{-1} d_{N T}$ for some constant $0<C_{\delta}^{\prime}<\infty$. Therefore, by following the procedure as the proof in Lemma $\mathbf{S . 1}$, we have

$$
\sup _{\left\|\boldsymbol{\lambda}_{j}-\boldsymbol{\lambda}_{j}^{0}\right\| \leq \widetilde{C} d_{N T}^{*}}\left|W_{N t j, 13}-E\left(W_{N t j, 13} \mid \mathbf{X}, \mathbf{F}\right)\right|=o_{p}\left(N^{-1 / 2}\right) .
$$

Therefore, the result in Lemma $\underline{S .6}$ is proved by (S.8), (S.9), (S.10) and (S.11).

Lemma S.7. Let Conditions (C1)-(C4) hold. If, in addition, $K_{N}^{4} N^{-1}=o(1), K_{N}^{-r+2}(\log T)=$ $o(1)$ and $K_{N}^{-1}(\log N T)(\log N)^{4}=o(1)$, then we have

$$
\left\|\widehat{\boldsymbol{\lambda}}^{[1]}-\boldsymbol{\lambda}^{0}-\Psi_{N T}^{-1} U_{N, 1}\right\|=O_{p}\left(d_{N T}\right)+o_{p}\left(N^{-1 / 2}\right),
$$

where $U_{N T, 1}$ is defined in (A.31) and $\Psi_{N T}$ is defined in (A.30).

Proof. By Lemma 4 and (A.20), we have $\left\|\widehat{f}_{t}^{[0]}-f_{t}^{0}\right\| \leq C_{f}\left(d_{N T}+N^{-1 / 2}\right)$ for some constant $0<C_{f}<\infty$. Let $Q_{i t}=\left\{B_{j}\left(X_{j i}\right)^{\top} f_{j t}, 1 \leq j \leq J\right\}^{\top}$. Let $f=\left(f_{1}^{\top}, \ldots, f_{T}^{\top}\right)^{\top}$ satisfy that $\left\|f_{t}-f_{t}^{0}\right\| \leq C_{f}\left(d_{N T}+N^{-1 / 2}\right)$. Write

$$
\begin{aligned}
& L_{N T}^{*}(f, \boldsymbol{\lambda}) \\
& =E\left\{L_{N T}^{*}(f, \boldsymbol{\lambda}) \mid \mathbf{X}, \mathbf{F}\right\}-\left(\boldsymbol{\lambda}-\boldsymbol{\lambda}^{0}\right)^{\top}\left\{V_{N T, 1}(f)-E\left(V_{N T, 1}(f) \mid \mathbf{X}, \mathbf{F}\right)\right\} \\
& +V_{N T, 2}(f, \boldsymbol{\lambda})-E\left(V_{N T, 2}(f, \boldsymbol{\lambda}) \mid \mathbf{X}, \mathbf{F}\right),
\end{aligned}
$$

where

$$
\begin{aligned}
V_{N T, 1}(f) & =(N T)^{-1} \sum_{i=1}^{N} \sum_{t=1}^{T} Q_{i t} \psi_{\tau}\left(y_{i t}-f_{u t}-\boldsymbol{\lambda}^{0 \top} Q_{i t}\right), \\
V_{N T, 2}(f, \boldsymbol{\lambda}) & =(N T)^{-1} \sum_{i=1}^{N} \sum_{t=1}^{T}\left\{\rho_{\tau}\left(y_{i t}-f_{u t}-\boldsymbol{\lambda}^{\top} Q_{i t}\right)-\rho_{\tau}\left(y_{i t}-f_{u t}-\boldsymbol{\lambda}^{0 \top} Q_{i t}\right)\right. \\
& \left.+\left(\boldsymbol{\lambda}-\boldsymbol{\lambda}^{0}\right)^{\top} Q_{i t} \psi_{\tau}\left(y_{i t}-f_{u t}-\boldsymbol{\lambda}^{0 \top} Q_{i t}\right)\right\} .
\end{aligned}
$$

By following the same reasoning as in the proofs of Lemmas S.4 and S.5, we have

$$
\begin{gathered}
E\left\{L_{N T}^{*}(f, \boldsymbol{\lambda}) \mid \mathbf{X}\right\}=-\left(\boldsymbol{\lambda}-\boldsymbol{\lambda}^{0}\right)^{\top} E\left(V_{N T, 1}(f) \mid \mathbf{X}, \mathbf{F}\right)+\frac{1}{2}\left(\boldsymbol{\lambda}-\boldsymbol{\lambda}^{0}\right)^{\top} \Psi_{N T}\left(\boldsymbol{\lambda}-\boldsymbol{\lambda}^{0}\right)+o_{p}\left(\left\|\boldsymbol{\lambda}-\boldsymbol{\lambda}^{0}\right\|^{2}\right), \\
V_{N T, 2}(f, \boldsymbol{\lambda})-E\left(V_{N T, 2}(f, \boldsymbol{\lambda}) \mid \mathbf{X}, \mathbf{F}\right)=o_{p}\left(\left\|\boldsymbol{\lambda}-\boldsymbol{\lambda}^{0}\right\|^{2}+(N T)^{-1}\right),
\end{gathered}
$$

uniformly in $\left\|f_{t}-f_{t}^{0}\right\| \leq C_{f}\left(d_{N T}+N^{-1 / 2}\right)$ and $\left\|\boldsymbol{\lambda}-\boldsymbol{\lambda}^{0}\right\| \leq \varsigma_{N T}$, where $\varsigma_{N T}$ is any sequence of positive numbers satisfying $\varsigma_{N T}=o(1)$. Thus, by (S.12), (S.14) and (S.15), we have

$$
L_{N T}^{*}(f, \boldsymbol{\lambda})=-\left(\boldsymbol{\lambda}-\boldsymbol{\lambda}^{0}\right)^{\top} V_{N T, 1}(f)+\frac{1}{2}\left(\boldsymbol{\lambda}-\boldsymbol{\lambda}^{0}\right)^{\top} \Psi_{N T}\left(\boldsymbol{\lambda}-\boldsymbol{\lambda}^{0}\right)+o_{p}\left(\left\|\boldsymbol{\lambda}-\boldsymbol{\lambda}^{0}\right\|^{2}+(N T)^{-1}\right),
$$


uniformly in $\left\|f_{t}-f_{t}^{0}\right\| \leq C_{f}\left(d_{N T}+N^{-1 / 2}\right)$ and $\left\|\boldsymbol{\lambda}-\boldsymbol{\lambda}^{0}\right\| \leq \varsigma_{N T}$. Therefore, we have

$$
\widehat{\lambda}^{[1]}-\lambda^{0}=\Psi_{N T}^{-1} V_{N T, 1}\left(\widehat{f}^{[0]}\right)+o_{p}\left\{(N T)^{-1 / 2}\right\} .
$$

By following the same reasoning as the proof for (A.3), as $(N, T) \rightarrow \infty$ with probability approaching 1, we have $\left\|\Psi_{N T}^{-1}\right\| \leq C_{\Psi}^{\prime}$ for some constant $0<C_{\Psi}^{\prime}<\infty$. In Lemma [S.8, we will show that $\left\|V_{N T, 1}\left(\widehat{f}^{[0]}\right)-U_{N T, 1}\right\|=O_{p}\left(d_{N T}\right)+o_{p}\left(N^{-1 / 2}\right)$. Therefore, the result in Lemma S.7 follows from the above results, and thus the proof is completed.

Lemma S.8. Let Conditions (C1)-(C4) hold. If, in addition, $K_{N}^{4} N^{-1}=o(1), K_{N}^{-r+2}(\log T)=$ $o(1)$ and $K_{N}^{-1}(\log N T)(\log N)^{4}=o(1)$, then we have

$$
\left\|V_{N T, 1}\left(\widehat{f}^{[0]}\right)-U_{N T, 1}\right\|=O_{p}\left(d_{N T}\right)+o_{p}\left(N^{-1 / 2}\right),
$$

where $V_{N T, 1}$ and $U_{N T, 1}$ are defined in (S.13) and (A.31), respectively.

Proof. Write

$$
V_{N T, 1}(f)=V_{N T, 11}+V_{N T, 12}(f)+V_{N T, 13}(f)
$$

where

$$
\begin{aligned}
V_{N T, 11} & =U_{N T, 1}=(N T)^{-1} \sum_{i=1}^{N} \sum_{t=1}^{T} Q_{i t}^{0} \psi_{\tau}\left(\varepsilon_{i t}\right), \\
V_{N T, 12}(f) & \left.=(N T)^{-1} \sum_{i=1}^{N} \sum_{t=1}^{T}\left(Q_{i t}-Q_{i t}^{0}\right) \psi_{\tau}\left(\varepsilon_{i t}\right)\right), \\
V_{N T, 13}(f) & \left.=(N T)^{-1} \sum_{i=1}^{N} \sum_{t=1}^{T} Q_{i t}\left\{\psi_{\tau}\left(y_{i t}-f_{u t}-\lambda^{0 \top} Q_{i t}\right)\right)-\psi_{\tau}\left(\varepsilon_{i t}\right)\right\} .
\end{aligned}
$$

Since $\left\|N^{-1} \sum_{i=1}^{N} B\left(X_{i}\right) \psi_{\tau}\left(\varepsilon_{i t}\right)\right\|=O_{p}\left(N^{-1 / 2}\right)$, we have with probability approaching 1,

$$
\begin{gathered}
\sup _{\left\|f_{t}-f_{t}^{0}\right\| \leq C_{f}\left(d_{N T}+N^{-1 / 2}\right)}\left\|V_{N T, 12}\right\| \leq T^{-1} \sum_{t=1}^{T}\left\|N^{-1} \sum_{i=1}^{N} B\left(X_{i}\right) \psi_{\tau}\left(\varepsilon_{i t}\right)\right\| \\
\times \quad \sup _{\left\|f_{t}-f_{t}^{0}\right\| \leq C_{f}\left(d_{N T}+N^{-1 / 2}\right)}\left\|f_{t}-f_{t}^{0}\right\|=O\left\{N^{-1 / 2}\left(d_{N T}+N^{-1 / 2}\right)\right\}=o\left(N^{-1 / 2}+d_{N T}\right) .
\end{gathered}
$$

By following the same procedure as the proof for (A.36), we have for any vector $\mathbf{a} \in R^{K_{N} J}$ with $\|\mathbf{a}\|=1$,

$$
\operatorname{var}\left(\mathbf{a}^{\top} V_{N T, 13}(f) \mathbf{a}\right)=O\left\{K_{N}\left(d_{N T}+N^{-1 / 2}\right)(N T)^{-1}\right\},
$$

uniformly in $\left\|f_{t}-f_{t}^{0}\right\| \leq C_{f}\left(d_{N T}+N^{-1 / 2}\right)$. Then by the procedure as the proof in Lemma S.1, we have

$$
\begin{aligned}
& \sup _{\left\|f_{t}-f_{t}^{0}\right\| \leq C_{f}\left(d_{N T}+N^{-1 / 2}\right)}\left\|V_{N T, 13}(f)-E\left\{V_{N T, 13}(f)\right\}\right\|=O_{p}\left\{K_{N}^{1 / 2}\left(d_{N T}+N^{-1 / 2}\right)^{1 / 2}(N T)^{-1 / 2}\right\} \\
& =o_{p}\left(d_{N T}\right) \text {. }
\end{aligned}
$$

Hence,

$$
\left\|V_{N T, 13}\left(\widehat{f}^{[0]}\right)-E\left\{V_{N T, 13}\left(\widehat{f}^{[0]}\right)\right\}\right\|=o_{p}\left(d_{N T}\right) .
$$


Let

$$
\kappa_{i t}(f)=f_{u t}^{0}-f_{u t}+\sum_{j=1}^{J}\left(\widetilde{g}_{j}^{0}\left(X_{j i}\right)\left(f_{j t}^{0}-f_{j t}\right)+r_{j, i t}^{*}\right) .
$$

Then there exist constants $0<C, C^{\prime}<\infty$ such that

$$
\begin{aligned}
\left\|E\left\{V_{N T, 13}(f) \mid \mathbf{X}, \mathbf{F}\right\}\right\| & \leq C\left\|E\left[(N T)^{-1} \sum_{i=1}^{N} \sum_{t=1}^{T} B_{i}\left(X_{i}\right)\left\{I\left(\varepsilon_{i t} \leq 0\right)-I\left(\varepsilon_{i t} \leq \kappa_{i t}(f)\right)\right\} \mid \mathbf{X}, \mathbf{F}\right]\right\| \\
& \leq C^{\prime}\left\|(N T)^{-1} \sum_{i=1}^{N} \sum_{t=1}^{T} B_{i}\left(X_{i}\right) \kappa_{i t}(f) p_{i}\left(0 \mid X_{i}, f_{t}\right)\right\|
\end{aligned}
$$

uniformly in $\left\|f_{t}-f_{t}^{0}\right\| \leq C_{f}\left(d_{N T}+N^{-1 / 2}\right)$. Moreover, by (A.20) and Lemma 4, we have

$$
\begin{aligned}
& \|(N T)^{-1} \sum_{i=1}^{N} \sum_{t=1}^{T} B_{i}\left(X_{i}\right) \kappa_{i t}\left(\widehat{f}^{[0]}\right) p_{i}\left(0 \mid X_{i}, f_{t}\right) \\
& +(N T)^{-1} \sum_{i=1}^{N} \sum_{t=1}^{T} B_{i}\left(X_{i}\right) p_{i}\left(0 \mid X_{i}, f_{t}\right) \widetilde{g}^{0}\left(X_{i}\right)^{\top}\left[\Lambda_{N}^{-1}\left\{N^{-1} \sum_{i=1}^{N} G_{i}^{0}\left(X_{i}\right)\left(\tau-I\left(\varepsilon_{i t}<0\right)\right)\right\}\right] \| \\
& =O\left(d_{N T}\right)+o_{p}\left(N^{-1 / 2}\right) .
\end{aligned}
$$

Since $\left\|(N T)^{-1} \sum_{t=1}^{T} \sum_{i=1}^{N} G_{i}^{0}\left(X_{i}\right)\left(\tau-I\left(\varepsilon_{i t}<0\right)\right)\right\|=O_{p}\left\{(N T)^{-1 / 2}\right\}$, and

$$
\left\|(N T)^{-1} \sum_{i=1}^{N} \sum_{t=1}^{T} B_{i}\left(X_{i}\right) p_{i}\left(0 \mid X_{i}, f_{t}\right)\right\|=O_{p}(1)
$$

we have

$$
\begin{aligned}
& \left\|(N T)^{-1} \sum_{i=1}^{N} \sum_{t=1}^{T} B_{i}\left(X_{i}\right) p_{i}\left(0 \mid X_{i}, f_{t}\right) \widetilde{g}^{0}\left(X_{i}\right)^{\top}\left[\Lambda_{N}^{-1}\left\{N^{-1} \sum_{i=1}^{N} G_{i}^{0}\left(X_{i}\right)\left(\tau-I\left(\varepsilon_{i t}<0\right)\right)\right\}\right]\right\| \\
& =O_{p}\left\{(N T)^{-1 / 2}\right\} .
\end{aligned}
$$

Therefore, by (․19) and (S.20), we have with probability approaching 1,

$$
\left\|E\left\{V_{N T, 13}\left(\widehat{f}^{[0]}\right) \mid \mathbf{X}, \mathbf{F}\right\}\right\|=O\left(d_{N T}\right)+o\left(N^{-1 / 2}\right) .
$$

By (S.18) and (S.21), we have

$$
\left\|V_{N T, 13}\left(\widehat{f}^{[0]}\right)\right\|=O_{p}\left(d_{N T}\right)+o_{p}\left(N^{-1 / 2}\right) .
$$

Therefore, the result in Lemma $\underline{\mathrm{S} .8}$ follows from (S.16), (S.17), and (S.22) directly. 